\author{
Universidade de São Paulo \\ Instituto de Física
}

\title{
Interação onda-partícula: Ressonâncias, aceleração regular e controle do caos
}

\author{
Meirielen Caetano de Sousa
}

Orientador: Prof. Dr. Iberê Luiz Caldas

Tese de Doutorado apresentada ao Instituto de Física da Universidade de São Paulo para a obtenção do título de Doutor em Ciências

Banca Examinadora:

Prof. Dr. Iberê Luiz Caldas (Orientador, Instituto de Física da USP)

Prof. Dr. Zwinglio de Oliveira Guimarães Filho (Instituto de Física da USP)

Prof. Dr. Rafael Ribeiro Dias Vilela de Oliveira (Universidade Federal do ABC)

Prof. Dr. Ricardo Luiz Viana (Universidade Federal do Paraná)

Prof. Dr. José Danilo Szezech Júnior (Universidade Estadual de Ponta Grossa) 


\section{FICHA CATALOGRÁFICA}

\section{Preparada pelo Serviço de Biblioteca e Informação}

do Instituto de Física da Universidade de São Paulo

Sousa, Meirielen Caetano de

Interação onda-partícula: ressonâncias, aceleração regular e controle do caos. São Paulo, 2015.

Tese (Doutorado) - Universidade de São Paulo. Instituto de Física. Depto. Física Aplicada.

Orientador: Prof. Dr. Iberê Luiz Caldas

Área de Concentração: Física de Plasmas e Descargas Elétricas.

Unitermos: 1. Física de plasmas; 2. Sistemas dinâmicos; 3. Aceleração de partículas; 4. Caos (Sistemas dinâmicos); 5. Controle. 


\section{AGRADECIMENTOS}

A Deus, pelas grandes oportunidades oferecidas, pela força nos momentos difíceis e pelas alegrias ao longo deste doutorado.

Ao meu orientador, Prof. Dr. Iberê Luiz Caldas, pelo apoio e incentivo constantes, por estar sempre presente e disposto a ajudar, por tudo que me ensinou nos últimos anos.

Aos Profs. Drs. Fernanda Monti Steffens, Felipe Barbedo Rizzato, Renato Pakter e Alfredo Miguel Ozorio de Almeida pela colaboração em artigos que resultaram nesta tese. Nossas discussões sempre foram muito produtivas.

Ao Prof. Dr. Fabrice Doveil por ter me recebido durante um ano em sua equipe na Universidade de Aix-Marseille, França. Foi uma grande oportunidade realizar trabalhos experimentais em Marselha, além do estudo teórico feito em São Paulo.

Ao engenheiro Dr. Didier Guyomarc'h que me acompanhou e instruiu durante meu estágio de pesquisa no Laboratório de Física das Interações Iônicas e Moleculares da Universidade de Aix-Marseille.

A todos do Grupo Controle de Oscilações da Universidade de São Paulo pelo incentivo, conversas e discussões científicas: Alberto, Bruno, Celso, David, Dennis, Everton, Julio, Rafael, Rene e Zwinglio.

Aos funcionários administrativos do Instituto de Física da Universidade de São Paulo que foram sempre muito prestativos, especialmente a Lia do Departamento de Física Aplicada e os funcionários da Comissão de Pós-Graduação.

A todos das equipes Turbulência em Plasma e Dinâmica de Sistemas Complexos, aos funcionários administrativos do Laboratório de Física das Interações Iônicas e Moleculares da Universidade de Aix-Marseille.

A Daniel, Luciano e Paulo que trabalharam comigo em Marselha, pelos momentos de descontração quando estávamos todos em um país estrangeiro.

A FAPESP, CNPq e CAPES pelas bolsas de estudo concedidas e pelo auxílio financeiro para participação em reuniões científicas.

Para o final, reservo agradecimentos especiais aos meus pais Nelson e Leonice, aos meus irmãos Carlos Eduardo e Suelen e a minha cunhada Kelly. A presença, o apoio, o incentivo e a compreensão de vocês tornaram tudo mais fácil e até mesmo possível.

A todos os amigos e familiares que sempre torceram por mim. 

We especially need imagination in science. It is not all mathematics, nor all logic, but it is somewhat beauty and poetry.

Maria Mitchell 



\section{RESUMO}

Nesta tese é analisada a dinâmica de uma partícula relativística se movendo sob a influência de um campo magnético uniforme e uma onda eletrostática e estacionária dada na forma de pulsos periódicos. O mapa que descreve a evolução temporal do sistema é explícito e pode ser considerado como uma versão relativística e magnetizada do mapa padrão clássico. A posição aproximada dos pontos periódicos é calculada analiticamente e com essa informação é possível estudar as ressonâncias primárias. Para o sistema em estudo, observa-se que a maior parte das ressonâncias possui mais de uma cadeia de ilhas. Isso ocorre pois o sistema apresenta um número infinito de termos ressonantes com o mesmo número de rotação e que podem gerar ilhas na mesma posição do espaço de fases. Verifica-se que essa superposição de termos ressonantes faz com que o número de cadeias varie em função dos parâmetros da onda. Para valores de período ou número de onda suficientemente elevados, todas as ressonâncias primárias apresentam duas ou mais cadeias de ilhas no espaço de fases. As ilhas de ressonância primária são utilizadas nesta tese para acelerar partículas de forma regular. Em particular, considera-se a ressonância principal do sistema, para a qual a energia inicial da partícula pode estar muito próxima de sua energia de repouso se os parâmetros da onda forem adequados. Além disso, aplica-se um método de controle do caos para Hamiltonianas quase integráveis que consiste na adição de um termo de controle simples e com baixa amplitude ao sistema. Esse termo de controle cria toros invariantes em todo o espaço de fases que confinam as trajetórias caóticas em pequenas regiões, tornando a dinâmica controlada mais regular. Verifica-se numericamente que o termo de controle reduz drasticamente as regiões caóticas. Além disso, observa-se que o controle do caos e a consequente recuperação de trajetórias periódicas e quase periódicas no espaço de fases podem ser utilizados para melhorar o processo de aceleração regular de partículas.

Palavras-chave: interação onda-partícula, ressonâncias primárias, múltiplas cadeias isócronas, aceleração regular de partículas, controle de caos. 



\begin{abstract}
In this thesis, we analyze the dynamics of a relativistic particle moving under the influence of a uniform magnetic field and a stationary electrostatic wave given as a series of periodic pulses. The map that describes the time evolution of the system is explicit, and it can be considered as a magnetized relativistic version of the classical standard map. We calculate analytically the approximate position of the periodic points and we use this information to study the primary resonances. For the system under study, we observe that most of its resonances exhibit more than one island chain. It occurs because the system presents an infinite number of resonant terms with the same winding number that may generate islands in the same position of phase space. We verify that this superposition of resonant terms makes the number of chains vary as a function of the parameters of the wave. For sufficiently large values of the wave period or wave number, all the primary resonances present two or more island chains in phase space. We use the islands of primary resonances in this thesis to regularly accelerate particles. In particular, we consider the main resonance of the system, for which the initial energy of the particle can be very close to its rest energy if the parameters of the wave are adequate. Furthermore, we apply a method of control of chaos for nearintegrable Hamiltonians that consists in the addition of a simple control term with low amplitude to the system. This control term creates invariant tori in the whole phase space that confine the chaotic trajectories to small regions, making the controlled dynamics more regular. We verify numerically that the control term drastically reduces the chaotic regions. Moreover, we observe that the control of chaos and the consequent recovery of periodic and quasiperiodic trajectories in phase space can be used to improve the process of regular particle acceleration.
\end{abstract}

Keywords: wave-particle interaction, primary resonances, multiple isochronous chains, regular particle acceleration, control of chaos. 



\section{LISTA DE FIGURAS}

Figura 1 Representação esquemática da variação das grandezas $I$ e $\theta$ entre os instantes $t=n T$ e $t=(n+1) T$

Figura 2 Espaço de fases do sistema para $\varepsilon=0$

Figura 3 Espaço de fases do sistema para $T=2 \pi(1+1 / 15), k=2$ e a) $\varepsilon=2,5$

$$
\begin{aligned}
& \times 10^{-4} \text {; b) } \varepsilon=2,5 \times 10^{-2} \text {; c) } \varepsilon=0,125 \text {; d) } \varepsilon=0,200 \text {; e) } \varepsilon=0,250 \text {; } \\
& \text { f) } \varepsilon=0,375
\end{aligned}
$$

Figura 4 Ampliação das ilhas das ressonâncias a) $(1,1)$ e b) $(2,1)$ identificadas na Figura 3(a)

Figura 5 Ampliação da região próxima ao ponto hiperbólico indicado por uma cruz preta na Figura 3(b)

Figura 6 Ampliação de algumas cadeias de ilhas da Figura 3(c)

Figura 7 Gráficos de $G_{r, s}(\theta) \times \theta$ para as ressonâncias a) $(4,3)$ e b) $(2,1)$. Parâmetros utilizados: $k=2$ e $T=2 \pi(1+1 / 15)$

Figura 8 Espaço de fases do sistema para $T=2,5 \pi, k=5$ e $\varepsilon=0,006$

Figura 9 Espaço de fases na região da ressonância $(1,1)$ para $T=2,5 \pi, \varepsilon=0,006$ e a) $k=2$; b) $k=5$

Figura 10 Número de cadeias isócronas em função de $T$ e $k$ para as ressonâncias a) $(1,1)$, b) $(1,2)$, c) $(2,1)$ e d) $(4,3)$

Figura 11 Número de cadeias isócronas em função de $T$ e $k$ para as ressonâncias a) $(5,2)$ e b), c) $(8,15)$

Figura 12 Ampliação do espaço de fases das Figuras 3(b) e 3(d) na região da ressonância (1,1). Parâmetros utilizados: $T=2 \pi(1+1 / 15), \quad k=2 \quad \mathrm{e}$ a) $\varepsilon=2,5 \times 10^{-2}$; b) $\varepsilon=0,200$

Figura 13 Posição dos pontos hiperbólicos da ressonância $(1,1)$ indicados por uma cruz preta na Figura 12. Parâmetros utilizados: $T=2 \pi(1+1 / 15), k=2 \mathrm{e}$ a) $\varepsilon=2,5 \times 10^{-2}$; b) $\varepsilon=0,200$

Figura 14 Bifurcação com dobramento de período dos pontos elípticos da ressonância principal do sistema. Parâmetros utilizados: $T=2 \pi(1+1 / 15)$, 
$k=2$ e a) $\varepsilon=2,500$ (antes da bifurcação); b) $\varepsilon=2,750$ (depois da bifurcação) ................................................................................. 54

Figura 15 A curva tracejada corresponde aos parâmetros da onda para os quais os pontos elípticos da ressonância $(1,1)$ localizados em $\theta=0 ; \pi$ sofrem uma bifurcação com dobramento de período. A curva sólida representa os valores mínimos dos parâmetros da onda para os quais os pontos hiperbólicos da ressonância $(1,1)$ estão localizados sobre o eixo $I=0$. A região sombreada indica o intervalo de parâmetros para os quais é possível acelerar a partícula de forma regular nas ilhas da ressonância $(1,1)$

Figura 16 Espaço de fases do sistema a) sem o termo de controle e b) com a adição do termo de controle. Parâmetros utilizados: $T=2 \pi(1+1 / 15), k=2$ e $\varepsilon=0,200$

Figura 17 Toros invariantes separando as regiões de ressonância no espaço de fases do sistema controlado. Parâmetros utilizados: $T=2 \pi(1+1 / 15), k=2$ e $\varepsilon=0,200$

Figura 18 Espaço de fases do sistema perturbado apenas pelo termo de controle. Parâmetros utilizados: $T=2 \pi(1+1 / 15), k=2$ e $\varepsilon=0,200$

Figura 19 Estrutura de uma válvula de ondas progressivas (TWT)

Figura 20 Relação de dispersão teórica (curva azul) e dados experimentais (em vermelho) para a nova versão da estrutura de ondas lentas (EOL). A figura também mostra uma comparação com os pontos experimentais (em verde) obtidos para a versão anterior do TWT

Figura 21 Velocidades de a) fase e de b) grupo em função da frequência da onda que se propaga no TWT 


\section{SUMÁRIO}

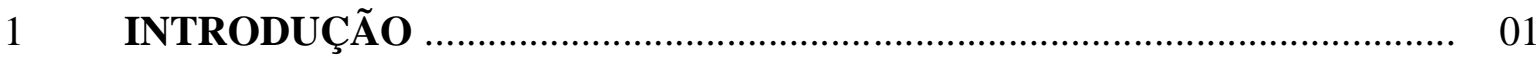

2 DESCRIÇÃO DO SISTEMA ………………......................................... 09

2.1 CONSTRUÇÃO DA HAMILTONIANA …………………………………...... 10

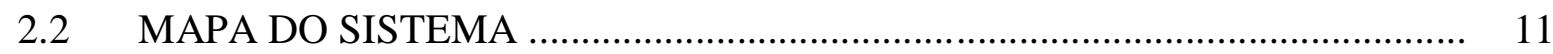

2.3 ESPAÇO DE FASES E TRANSIÇÃO PARA O CAOS ……………………….. 17

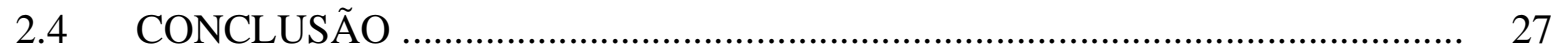

3 RESSONÂNCIAS PRIMÁRIAS E MÚLTIPLAS CADEIAS ISÓCRONAS 29

3.1 TEOREMA DO PONTO FIXO DE POINCARÉ-BIRKHOFF ........................... 30

3.2 POSIÇÃO DAS RESSONÂNCIAS PRIMÁRIAS COM RELAÇÃO À VARIÁVEL DE AÇÃO ................................................................................. 31

3.3 POSIÇÃO DAS RESSONÂNCIAS PRIMÁRIAS COM RELAÇÃO À VARIÁVEL ANGULAR ............................................................................... 33

3.4 NÚMERO DE CADEIAS ISÓCRONAS PRESENTES NAS REGIÕES DE RESSONÂNCIAS PRIMÁRIAS .................................................................... 36

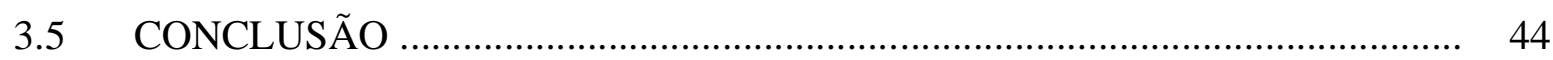

4 ACELERAÇÃO REGULAR DE PARTÍCULAS A PARTIR DE BAIXAS ENERGIAS INICIAIS .......................................................................... 4

4.1 POSIÇÃO DOS PONTOS FIXOS DA RESSONÂNCIA PRINCIPAL DO

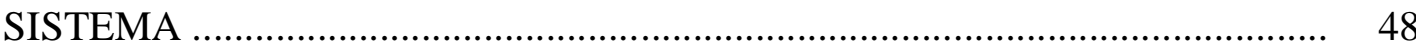

4.2 PONTOS HIPERBÓLICOS DA RESSONÂNCIA PRINCIPAL

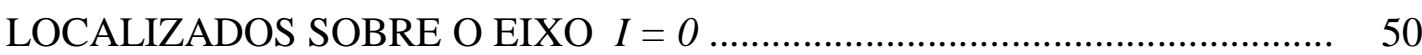

4.3 BIFURCAÇÃO DOS PONTOS ELÍPTICOS DA RESSONÂNCIA

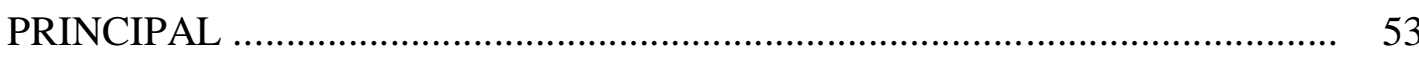

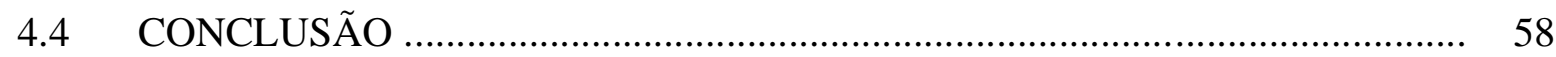

$5 \quad$ CONTROLE GLOBAL DO CAOS …………………………………..... 61

5.1 MÉTODO DE CONTROLE ................................................................... 61

5.2 APLICAÇÃO DO MÉTODO DE CONTROLE ................................................ 64

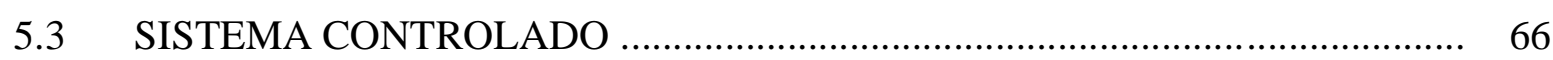

5.4 ACELERAÇÃO REGULAR DE PARTÍCULAS NO SISTEMA CONTROLADO 
5.5 COMPROVAÇÃO EXPERIMENTAL EM UMA VÁLVULA DE ONDAS

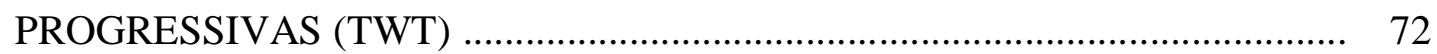

5.5.1 Montagem experimental e caracterização de uma válvula de ondas progressivas (TWT) ..................................................................... 73

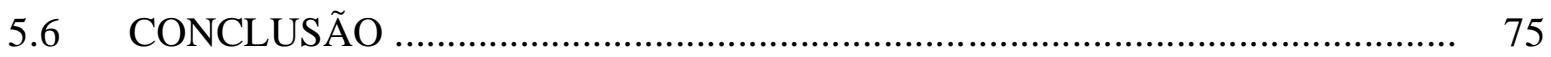

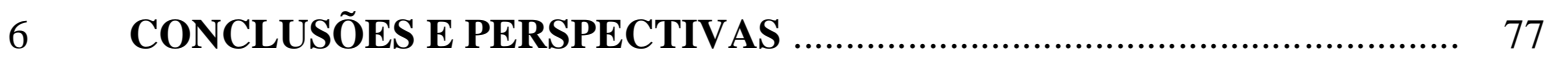

REFERÊNCIAS BIBLIOGRÁFICAS ……........................................ 83

APÊNDICE A - Propriedades de simetria da onda eletrostática e estacionária dada na forma de pulsos periódicos …....................................... $\quad 89$

APÊNDICE B - Pontos periódicos presentes em todas as ressonâncias primárias

APÊNDICE C - Intervalo de parâmetros para um dado número de cadeias de ilhas nas ressonâncias $(1, s)$ e $(2, s)$

APÊNDICE D - Relação de dispersão e velocidades de fase e de grupo para a nova válvula de ondas progressivas (TWT) do Laboratório de Física das Interações Iônicas e Moleculares (Laboratório PIIM) 


\section{INTRODUÇÃO}

Sistemas de interação onda-partícula sempre despertaram muita atenção por estarem presentes em diversas áreas, como por exemplo, em aceleradores de partículas [1-3], lasers de elétrons livres [3], na autorressonância ciclotrônica [3], em sistemas astrofísicos [3], na descrição da dinâmica microscópica do plasma [3], e na transmissão de correntes em dispositivos de fusão [4, 5]. Além disso, esse tipo de interação é utilizado em muitas aplicações como uma forma eficiente de aquecimento [3, 6-8] e aceleração de partículas [2, 3, $7,9,10]$.

Feixes de partículas aceleradas por ondas e confinadas por campos magnéticos são utilizados principalmente em aceleradores de partículas. Os progressos científicos e o rápido desenvolvimento do campo de aceleradores nas últimas décadas tornam cada vez mais importante o estudo da dinâmica de feixes e de mecanismos que contribuam para a aceleração regular de partículas, como realizado nesta tese.

Aceleradores de partículas possuem diversas aplicações na indústria, na medicina, em assuntos de segurança nacional, na geração de conhecimento sobre a estrutura da matéria, etc., como descrito com mais detalhes nas Refs. [2, 11, 12]. Entre as aplicações apresentadas nessas referências pode-se citar o uso de aceleradores em estudos que tentam compreender a massa, a matéria escura e a energia escura que formam o universo, em física nuclear e física de partículas, no tratamento de superfícies de materiais, na implantação de íons em semicondutores, na detecção de petróleo, no diagnóstico e tratamento de doenças (especialmente o câncer), na produção de radioisótopos para farmacoterapia, na datação e identificação de materiais arqueológicos, na inspeção de cargas em portos e aeroportos, etc.

A interação entre ondas e partículas, como observado, por exemplo, nos aceleradores de partículas, é basicamente um processo não linear [3, 13] que pode apresentar trajetórias regulares e caóticas em seu espaço de fases [14]. Trajetórias periódicas ou quase periódicas são úteis para aceleração coerente de partículas [15-17], enquanto trajetórias caóticas são responsáveis pelo aquecimento de partículas [6]. O tipo de trajetória que prevalece no espaço de fases depende principalmente da amplitude da perturbação aplicada ao sistema. De forma geral, o sistema se torna cada vez mais caótico à medida que a amplitude da perturbação aumenta. Mas para esse tipo de interação, o comportamento das partículas pode se tornar caótico mesmo quando a amplitude da onda é baixa. Essa característica faz com que seja importante controlar o caos no sistema, de forma que sua dinâmica seja regular 
para valores mais elevados da amplitude da onda.

Controle de caos é um desafio chave em muitas áreas da física [18-27] e diversos métodos foram desenvolvidos com o objetivo de controlar algumas trajetórias específicas no espaço de fases [20, 28-32]. Entretanto, esses métodos não são viáveis para sistemas de interação onda-partícula por causa do grande número de trajetórias a serem controladas simultaneamente. Há alguns anos, um método de controle baseado em teoria de perturbação e álgebra de Lie foi desenvolvido na Ref. [33] para sistemas conservativos que podem ser descritos por uma Hamiltoniana integrável perturbada por um termo de baixa amplitude. Esse método não busca controlar trajetórias específicas. Ele cria toros invariantes em todo o espaço de fases que previnem o surgimento de caos.

Contudo, o termo de controle utilizado para criar os toros invariantes no espaço de fases deve preencher algumas condições. Por questões energéticas, o termo de controle deve ser muito menor do que a perturbação originalmente aplicada ao sistema, isto é, o termo de controle deve modificar muito pouco o sistema, mas ser capaz de reduzir as regiões caóticas no espaço de fases. O termo de controle também deve ser o mais simples possível para que ele possa ser implementado em experimentos como na Ref. [34] em que um termo de controle foi utilizado para aumentar a coerência cinética de um feixe de elétrons se propagando em uma válvula de ondas progressivas [35, 36], ou TWT (do inglês, traveling wave tube).

Além desse experimento, o método de controle desenvolvido na Ref. [33] tem sido utilizado principalmente em estudos teóricos sobre o controle de transporte caótico em sistemas Hamiltonianos, como, por exemplo, em plasmas confinados por campos magnéticos [25, 37, 38], dispositivos de fusão [25] e campos elétricos turbulentos [39]. Realmente, para controlar o caos no sistema, esse método cria toros invariantes em todo o espaço de fases que dificultam o transporte caótico. Entretanto, a adição de toros invariantes ao sistema também restaura muitas de suas trajetórias regulares originais, que de outra forma teriam sido destruídas pelo caos. Em sistemas de interação onda-partícula, trajetórias periódicas e quase periódicas são responsáveis pela aceleração regular das partículas. Logo, a recuperação dessas trajetórias no espaço de fases é muito importante para o processo de aceleração regular.

Se a onda que perturba o movimento das partículas for dada na forma de pulsos periódicos, é possível construir um mapa a partir da Hamiltoniana que descreve o sistema. O mapa obtido é chamado de mapa simplético, pois ele mantém a forma canônica das equações de Hamilton, preservando a área no espaço de fases [14]. Mapas são muito úteis no estudo de sistemas conservativos, pois eles mantêm as características do sistema e são mais simples do 
que as equações diferenciais de movimento. Graças a essa simplicidade, os mapas permitem que uma série de cálculos analíticos sejam feitos com grande precisão, mesmo quando a dinâmica é não linear [14, 40, 41]. Além disso, a análise numérica é bem mais simples do que a feita para equações diferenciais.

Entre todos os mapas simpléticos, o mais estudado na literatura é o mapa padrão com um grau de liberdade e algumas de suas variações [42, 43]. O mapa padrão é um mapa não linear que possui apenas um parâmetro de controle. Variando esse parâmetro, observa-se a transição do sistema para o caos global. O mapa padrão descreve a dinâmica de vários sistemas, além de ser utilizado como uma aproximação local para a região da separatriz das ilhas de ressonância [14, 40].

Entre os sistemas que podem ser descritos através do mapa padrão, encontramse sistemas de interação onda-partícula nos quais as partículas se movem sob a ação de campos eletrostáticos ${ }^{1}$ dados na forma de pulsos periódicos [3, 13]. Nesse caso, uma das variáveis canônicas aparece apenas no termo perturbativo da Hamiltoniana e a outra variável aparece apenas no termo de energia cinética das partículas. Portanto, uma das variáveis permanece constante entre dois pulsos consecutivos do campo eletrostático, enquanto a outra variável não sofre alterações durante um pulso. Essa característica simplifica os cálculos e faz com que o mapa padrão seja linear com relação à amplitude da onda.

Por outro lado, se as partículas também estiverem sob a ação de um campo magnético uniforme, sua energia cinética será função das duas variáveis canônicas. Nesse caso, apenas o momento das partículas sofre uma mudança abrupta durante os pulsos do campo eletrostático. Mas entre dois pulsos consecutivos, nenhuma das variáveis será conservada. Embora os cálculos se tornem mais complicados nesse caso, ainda é possível obter um mapa exato utilizando transformações canônicas adequadas, como pode ser visto ao longo desta tese e nas Refs. [16, 17].

No caso de interações onda-partícula como as descritas nos parágrafos anteriores, observa-se a partir do mapa do sistema que condições de ressonância apropriadas criam mecanismos eficientes para a aceleração [16, 17] e o aquecimento de partículas [44]. Nesta tese, será analisada a aceleração regular de partículas que ocorre quando as ondas eletrostáticas se propagam na direção perpendicular ao campo magnético uniforme, assim como descrito na Ref. [16]. Esses modos estão presentes em diversos sistemas e podem

\footnotetext{
${ }^{1}$ Campos eletrostáticos são campos elétricos dependentes do tempo cujo rotacional é nulo. Nesse caso, apesar da dependência temporal, o campo elétrico pode ser obtido a partir de um potencial elétrico e o campo magnético associado não varia no tempo.
} 
fornecer uma grande quantidade de energia para as partículas.

Em sistemas abertos, ondas eletrostáticas progressivas são responsáveis pela aceleração coerente $[6,7,10]$, assim como pelo aquecimento incoerente de partículas [8]. Em sistemas fechados, esses processos são gerados por modos estacionários compostos por ondas de mesma amplitude e mesma frequência que se propagam em sentidos opostos. Sistemas fechados incluem, por exemplo, feixes de partículas e colunas de plasma confinados magneticamente e a frequência da onda pode estar próxima da frequência ciclotrônica [45, 46]. Se as partículas do feixe ou do plasma forem relativísticas, seu movimento transversal ao campo magnético será não linear. Isso cria grandes ilhas de ressonância no espaço de fases $[47,48]$ através das quais é possível acelerar as partículas de forma regular.

Ao longo desta tese, será analisada a dinâmica de um feixe de partículas relativísticas se movendo sob a ação combinada de um campo magnético uniforme e uma onda eletrostática e estacionária que se propaga na direção perpendicular ao campo magnético. No caso em que a densidade do feixe é baixa, é possível afirmar que o feixe não altera a propagação da onda e suas partículas podem ser consideradas como partículas de teste que não interagem umas com as outras. Logo, o principal efeito é a interação individual de cada partícula do feixe com o campo magnético e a onda eletrostática, como é considerado nesta tese.

Em um trabalho anterior [49], o comportamento do sistema descrito acima foi analisado considerando partículas não relativísticas. O caso relativístico também foi abordado na Ref. [49], mas as aproximações utilizadas são válidas apenas para baixas amplitudes de onda. Nesta tese, a evolução temporal do sistema relativístico é resolvida de forma exata e pode ser analisada para qualquer valor da amplitude da onda eletrostática. Além disso, a utilização de expressões exatas para descrever o sistema permite a obtenção de uma série de novos resultados. Grande parte dos resultados descritos nesta tese foram publicados em periódicos internacionais e podem ser encontrados nas Refs. [16, 17, 50, 51]. A Ref. [44] contém resultados que não são apresentados nesta tese e que foram obtidos para o caso em que onda eletrostática se propaga em uma direção arbitrária com relação ao campo magnético. Além disso, foi realizado um trabalho experimental a partir de uma válvula de ondas progressivas [35, 36], ou TWT (do inglês, traveling wave tube), que simula a interação ondapartícula. A parte experimental do trabalho realizado nesta tese foi apresentada em conferências e publicada nos anais dos eventos, como pode ser visto nas Refs. [52, 53].

No modelo desenvolvido nesta tese, considera-se uma onda eletrostática dada na forma de pulsos que perturbam o sistema em intervalos de tempo periódicos. Perturbações 
impulsivas permitem a integração analítica da Hamiltoniana entre pulsos consecutivos e é possível obter um mapa de Poincaré, ou mapa estroboscópico, exato e completamente explícito que descreve a evolução temporal do sistema. A partir do mapa de Poincaré, é possível construir o espaço de fases do sistema para diversos valores dos parâmetros da onda eletrostática. Dessa forma, observa-se para quais parâmetros o comportamento do sistema é regular e como ocorre a transição para o caos à medida que os parâmetros da onda aumentam.

O mapa obtido pode ser considerado como uma versão relativística e magnetizada do mapa padrão clássico. Embora relacionados, será visto que o mapa magnetizado é não linear com relação à amplitude da onda. Isso faz com que os pontos periódicos das ressonâncias sejam deslocados pelo aumento da amplitude da onda, modificando a estrutura do espaço de fases.

Em geral, encontra-se na literatura sistemas que apresentam apenas uma cadeia de ilhas nas regiões de ressonância $[14,54]$. Entretanto, será visto que a maior parte das ressonâncias do sistema em estudo apresenta mais de uma cadeia de ilhas no espaço de fases $[50,51]$. Observa-se também que o número de cadeias aumenta com o número de onda e com o período da onda eletrostática. Para valores suficientemente elevados do período e/ou do número de onda, todas as ressonâncias do sistema possuem mais de uma cadeia de ilhas. Esse fenômeno ocorre pois a onda que perturba o sistema apresenta um número infinito de termos ressonantes com o mesmo número de rotação e que podem gerar ilhas de ressonância na mesma região do espaço de fases [50, 51]. A superposição de termos ressonantes com o mesmo número de rotação faz com que o número de cadeias de ilhas varie em função dos parâmetros do sistema.

Outra característica importante do modelo desenvolvido é que todas as ressonâncias apresentam um número par de ilhas no espaço de fases [50]. Devido a essa propriedade de simetria, todas as cadeias que possuem um número ímpar de ilhas ocorrem aos pares no espaço de fases. Para as ressonâncias que possuem um número par de ilhas em cada cadeia, o número de cadeias no espaço de fases pode ser tanto par quanto ímpar.

O estudo das ressonâncias primárias do sistema é de extrema importância, pois nas ilhas de ressonância, a onda eletrostática transfere uma grande quantidade de energia para as partículas e elas podem ser aceleradas de forma regular [15-17]. As Refs. [15, 16] determinam os valores dos parâmetros da onda para os quais a aceleração pode ser máxima. Entretanto, o processo de aceleração regular também depende da trajetória seguida pelas partículas. Para atingir a condição de máxima aceleração, é necessário conhecer a posição das ressonâncias e o número de cadeias de ilhas no espaço de fases em função dos parâmetros do 
sistema. Dessa forma, é possível ajustar as condições iniciais das partículas e fazer com elas sigam a melhor trajetória no espaço de fases.

Nesta tese será dada atenção especial à ressonância principal do sistema, para a qual a energia inicial das partículas pode estar próxima de sua energia de repouso quando o período da onda é ligeiramente maior do que o período ciclotrônico [16]. Este valor de período é escolhido pois diversos sistemas possuem essa característica, como pode ser visto nas Refs. [45, 46]. Quando o período da onda é próximo do período cliclotrônico, o aumento da amplitude da onda provoca o deslocamento dos pontos hiperbólicos da ressonância principal, fazendo com que eles atinjam o nível de energia mínima que corresponde à energia de repouso das partículas [16]. Por causa do deslocamento dos pontos hiperbólicos, as ilhas da ressonância principal passam a apresentar uma forma triangular, melhorando o processo de aceleração regular.

Entretanto, existe um limite para esse processo. Quando os parâmetros da onda eletrostática são elevados o suficiente, os pontos elípticos da ressonância principal perdem estabilidade e se tornam hiperbólicos. As ilhas de ressonância deixam de existir e não é mais possível acelerar as partículas de forma regular. Comparando os parâmetros da onda que provocam a bifurcação dos pontos elípticos e os parâmetros que deslocam os pontos hiperbólicos até o nível de energia mínima, obtém-se um intervalo de parâmetros da onda eletrostática para os quais é possível acelerar as partículas de forma regular nas ilhas da ressonância principal [16].

Além disso, será utilizado o método de controle desenvolvido na Ref. [33] e apresentado nas Refs. [34, 37, 38] para controlar o caos no sistema. Esse método de controle consiste na adição de uma perturbação com baixa amplitude à Hamiltoniana. Para o sistema em estudo, verifica-se que essa perturbação é simplesmente uma segunda onda eletrostática e estacionária com amplitude muito menor do que a amplitude da onda original [17]. A adição dessa segunda onda ao sistema não altera as suas estruturas principais, mas torna a dinâmica regular para valores mais elevados da amplitude da onda original.

Ao regularizar o sistema, o controle do caos restaura diversas órbitas periódicas e quase periódicas no espaço de fases, melhorando a aceleração regular que as partículas experimentam nessas trajetórias [17]. Isso significa que a energia inicial das partículas pode ser menor na dinâmica controlada do que ela deve ser no sistema sem controle e ainda assim, sua energia final será maior do que no sistema original.

No Capítulo 2 desta tese, são obtidos a Hamiltoniana e o mapa que descrevem o sistema em estudo [16, 17]. Verificam-se os diferentes tipos de comportamento 
apresentados pelo sistema e como ocorre a transição para o caos à medida que a amplitude da perturbação aumenta. No Capítulo 3, calcula-se analiticamente a posição dos pontos periódicos das ressonâncias primárias. A partir das expressões obtidas, observa-se que a maior parte das ressonâncias apresenta mais de uma cadeia de ilhas no espaço de fases [50, 51]. Além disso, o número de cadeias aumenta sem limites com o período e o número de onda para todas as ressonâncias primárias.

No Capítulo 4, é realizado um estudo sobre a aceleração regular de partículas a partir de baixas energias iniciais [16]. Verifica-se que a ressonância principal do sistema apresenta uma forma triangular, o que melhora o processo de aceleração e permite que a energia inicial da partícula esteja próxima de sua energia de repouso. No Capítulo 5, aplica-se ao sistema em estudo o método de controle do caos desenvolvido na Ref. [33]. Verifica-se numericamente que a adição de um termo de controle com baixa amplitude ao sistema reduz drasticamente as regiões de comportamento caótico, além de contribuir para a aceleração regular de partículas [17]. Neste capítulo também é apresentada uma montagem experimental capaz de simular a interação onda-partícula. $\mathrm{O}$ equipamento foi utilizado para verificar experimentalmente a possibilidade de controlar o caos nesse tipo de interação [34]. No Capítulo 6, são apresentadas as conclusões e perspectivas de continuação deste trabalho. 


\section{DESCRIÇÃO DO SISTEMA}

Ao longo desta tese, será analisado o comportamento de um feixe de partículas carregadas interagindo com um campo magnético e uma onda eletrostática, assim como descrito nas Refs. [16, 17]. Campos magnéticos externos são encontrados em diversos sistemas de interação onda-partícula, como por exemplo em sistemas astrofísicos. Além disso, campos magnéticos são úteis em experimentos, pois eles restringem o movimento das partículas, confinando-as em uma região limitada do espaço.

No caso em que a densidade do feixe é muito baixa, é possível afirmar que o feixe não altera a propagação da onda. Além disso, as partículas em um feixe de baixa densidade podem ser consideradas como partículas de teste que não interagem umas com as outras. Isso equivale a dizer que em um feixe de baixa densidade, os campos elétrico e magnético produzidos pelo movimento das partículas do feixe podem ser desprezados pois suas intensidades são muito menores do que a intensidade do campo elétrico associado à onda eletrostática e a intensidade do campo magnético externo. Logo, o principal efeito é a interação individual de cada partícula do feixe com o campo magnético externo e a onda eletrostática que perturba o feixe, como é considerado a seguir para a dedução da Hamiltoniana do sistema.

Quando a onda eletrostática é dada na forma de pulsos periódicos, é obtido um mapa exato que descreve a evolução temporal do sistema [16, 17]. A partir do mapa são construídos espaços de fases para diversos valores dos parâmetros da onda. Os espaços de fases nos permitem analisar o comportamento do sistema, os diferentes tipos de trajetórias e como órbitas regulares se tornam caóticas à medida que a perturbação aplicada ao sistema aumenta.

Órbitas caóticas estão fortemente relacionadas à presença de ressonâncias não lineares no espaço de fases. Essas ressonâncias surgem devido à interação da partícula com a onda eletrostática. Como será discutido a seguir, quanto maior a perturbação provocada pela onda, maior é a largura das ilhas de ressonância, o que provoca alterações drásticas na estrutura do espaço de fases e contribui para o surgimento de trajetórias caóticas. 


\subsection{CONSTRUÇÃO DA HAMILTONIANA}

Seja uma partícula relativística com carga $q$ e massa de repouso $m$ se movendo sob a ação de um campo magnético externo e uniforme $\vec{B}=B_{0} \hat{z}$ cujo potencial vetor é $\vec{A}=B_{0} x \hat{y}$. A Hamiltoniana que descreve esse sistema equivale à energia relativística da partícula

$$
H_{0}=\sqrt{m^{2} c^{4}+c^{2}|\vec{p}-q \vec{A}|^{2}}
$$

onde $c$ é a velocidade da luz no vácuo. Além disso, o momento canônico de uma partícula relativística se movendo em um campo magnético é dado por

$$
\vec{p}=m \gamma \vec{v}+q \vec{A}
$$

com $\gamma=\left(1-v^{2} / c^{2}\right)^{-1 / 2}$ e $\vec{v}$ é a velocidade da partícula. Considerando apenas a dinâmica transversal a $\vec{B}$, a Hamiltoniana (1) torna-se

$$
H_{0}=\sqrt{m^{2} c^{4}+c^{2} p_{x}^{2}+c^{2}\left(p_{y}-q B_{0} x\right)^{2}} .
$$

Suponha que a partícula também interage com um pacote de ondas planas, eletrostáticas $^{2}$ e estacionárias que se propagam em uma direção perpendicular ao campo magnético $\vec{B}$. Essas ondas possuem vetor de onda $\vec{k}=k \hat{x}$, período $T$ e amplitude $\varepsilon / 2$. A energia potencial devido às ondas eletrostáticas e estacionárias é dada por ${ }^{3}$

$$
U=\varepsilon V=\frac{q \varepsilon}{2} \cos (k x) \sum_{n=-\infty}^{+\infty} \delta(t-n T)
$$

onde $V$ é o potencial elétrico e a dependência temporal das ondas foi dada na forma de pulsos periódicos representados pela soma periódica de funções delta.

Dessa forma, a Hamiltoniana que descreve todas as interações do sistema é obtida através da soma das expressões (3) e (4) [16, 17]

\footnotetext{
${ }^{2}$ Ondas eletrostáticas são ondas que apresentam uma dependência temporal de tal forma que $\vec{\nabla} \times \vec{E}(t)=0$, onde $\vec{E}(t)$ é o campo elétrico associado à onda. Como $\vec{\nabla} \times \vec{E}(t)=0, \partial \vec{B} / \partial t=0$ e o campo elétrico pode ser obtido a partir de um potencial elétrico $V(t)$ na forma $\vec{E}(t)=-\vec{\nabla} V(t)$.

${ }^{3}$ A expressão (4) para a energia potencial não apresenta condições de contorno pois as ondas são planas.
} 


$$
H=H_{0}+\varepsilon V=\sqrt{m^{2} c^{4}+c^{2} p_{x}^{2}+c^{2}\left(p_{y}-q B_{0} x\right)^{2}}+\frac{q \varepsilon}{2} \cos (k x) \sum_{n=-\infty}^{+\infty} \delta(t-n T) .
$$

Como a Hamiltoniana (5) não é função da variável $y$, tem-se que $\dot{p}_{y}=-\partial H / \partial y=0$. Portanto, $p_{y}$ é uma constante de movimento e por simplicidade será considerado, sem perda de generalidade, $p_{y}=0$. Entretanto, é importante ressaltar que essa condição não é uma restrição física real, pois embora $p_{y}$ seja conservado e considerado igual a zero, $\mathrm{d} y / \mathrm{d} t$ não é nulo e a partícula descreve um movimento bidimensional no plano $x \mathrm{O} y$.

Trabalhando com as quantidades adimensionais $p_{x} / m c \rightarrow p_{x}, q B_{0} x / m c \rightarrow x$, $m c k / q B_{0} \rightarrow k, \quad q B_{0} t / m \rightarrow t, \quad q B_{0} T / m \rightarrow T \quad$ e $\quad\left(q^{2} B_{0} / m^{2} c^{2}\right) \varepsilon \rightarrow \varepsilon, \quad$ a Hamiltoniana adimensional $H / m c^{2} \rightarrow H$ que descreve o sistema é dada por $[16,17]$

$$
H\left(x, p_{x}, t\right)=H_{0}\left(x, p_{x}\right)+\varepsilon V(x, t)=\sqrt{1+p_{x}^{2}+x^{2}}+\frac{\varepsilon}{2} \cos (k x) \sum_{n=-\infty}^{+\infty} \delta(t-n T) .
$$

A partir da Hamiltoniana (6), observa-se que a cada vez que $t=n T$, a perturbação provocada pela onda se ativa por um instante infinitesimal. Entretanto, entre dois pulsos de onda consecutivos a partícula só está sujeita à ação do campo magnético e a Hamiltoniana que descreve o sistema torna-se integrável e independe do tempo. Logo, é possível escrevê-la em termos de suas variáveis de ângulo-ação através da mudança de coordenadas $x=\sqrt{2 I} \operatorname{sen} \theta$ e $p_{x}=\sqrt{2 I} \cos \theta$. Em termos das variáveis $(I, \theta)$, a Hamiltoniana (6) é reescrita como $[16,17]$

$$
H(I, \theta, t)=H_{0}(I)+\varepsilon V(I, \theta, t)=\sqrt{1+2 I}+\frac{\varepsilon}{2} \cos (k \sqrt{2 I} \operatorname{sen} \theta) \sum_{n=-\infty}^{+\infty} \delta(t-n T) .
$$

\subsection{MAPA DO SISTEMA}

A partir da Hamiltoniana (7), é possível obter um mapa que descreve a evolução temporal do sistema. Para obtenção desse mapa será utilizado o esquema apresentado na Figura 1. Nessa figura, $I_{n}$ e $\theta_{n}$ são os valores de $I$ e $\theta$ à esquerda, ou seja, imediatamente antes da função delta centrada em $t=n T$. Na travessia dessa função delta, as 
grandezas $I$ e $\theta$ sofrem uma variação representada por $\Delta_{1} I$ e $\Delta_{1} \theta$, atingindo assim os valores $I_{n}^{+}$e $\theta_{n}^{+}$à direta, ou seja, imediatamente após a função delta centrada em $t=n T$. Entre a direita da função delta centrada em $t=n T$ e a esquerda da função delta centrada em $t=(n+1) T$, não existem pulsos de onda e as grandezas $I$ e $\theta$ sofrem uma variação dada por $\Delta_{2} I$ e $\Delta_{2} \theta$, atingindo dessa forma os valores $I_{n+1}$ e $\theta_{n+1}$ imediatamente antes da função delta centrada em $t=(n+1) T$.

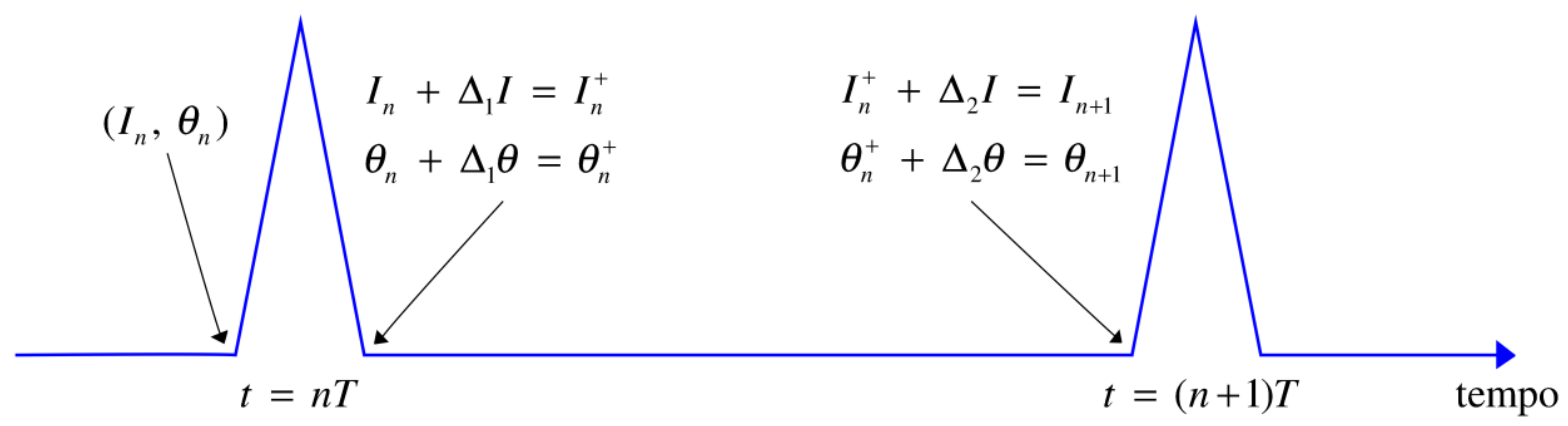

Figura 1: Representação esquemática da variação das grandezas $I$ e $\theta$ entre os instantes $t=n T$ e $t=(n+1) T$.

Antes de calcular as variações sofridas pelas grandezas $I$ e $\theta$ entre dois pulsos de onda consecutivos, é conveniente observar que o segundo termo da Hamiltoniana (7) depende das duas variáveis do sistema $I$ e $\theta$. Entretanto, o segundo termo da Hamiltoniana (6) depende apenas da variável $x$ e, portanto, apenas o momento $p_{x}$ sofre uma mudança abrupta quando $t=n T$. Por esse motivo, é mais simples utilizar a Hamiltoniana (6) para calcular as variações sofridas por $x$ e $p_{x}$ na travessia da função delta centrada em $t=n T$,

$$
\begin{aligned}
& \Delta_{1} x=\int_{n T-0}^{n T+0} \frac{\mathrm{d} x}{\mathrm{~d} t} \mathrm{~d} t=\int_{n T-0}^{n T+0} \frac{\partial H}{\partial p_{x}} \mathrm{~d} t=\int_{n T-0}^{n T+0} \frac{p_{x}}{\sqrt{1+p_{x}^{2}+x^{2}}} \mathrm{~d} t \\
& \Delta_{1} x \cong\left\langle\frac{p_{x}}{\sqrt{1+p_{x}^{2}+x^{2}}}\right\rangle \int_{n T-0}^{n T+0} \mathrm{~d} t \rightarrow 0, \\
& \Delta_{1} p_{x}=\int_{n T-0}^{n T+0} \frac{\mathrm{d} p_{x}}{\mathrm{~d} t} \mathrm{~d} t=-\int_{n T-0}^{n T+0} \frac{\partial H}{\partial x} \mathrm{~d} t \\
& \Delta_{1} p_{x}=-\int_{n T-0}^{n T+0}\left[\frac{x}{\sqrt{1+p_{x}^{2}+x^{2}}}-\frac{\varepsilon k}{2} \operatorname{sen}(k x) \delta(t-n T)\right] \mathrm{d} t
\end{aligned}
$$




$$
\begin{aligned}
& \Delta_{1} p_{x} \cong-\left\langle\frac{x}{\sqrt{1+p_{x}^{2}+x^{2}}}\right\rangle \int_{n T-0}^{n T+0} \mathrm{~d} t+\int_{n T-0}^{n T+0} \frac{\varepsilon k}{2} \operatorname{sen}(k x) \delta(t-n T) \mathrm{d} t, \\
& \Delta_{1} p_{x} \cong 0+\int_{n T-0}^{n T+0} \frac{\varepsilon k}{2} \operatorname{sen}(k x) \delta(t-n T) \mathrm{d} t,
\end{aligned}
$$

onde foram utilizadas as equações de Hamilton na segunda passagem de cada expressão e as integrais $\int_{n T-0}^{n T+0} f\left(x, p_{x}\right) \mathrm{d} t$ foram aproximadas por $\left\langle f\left(x, p_{x}\right)\right\rangle \int_{n T-0}^{n T+0} \mathrm{~d} t$, sendo $\left\langle f\left(x, p_{x}\right)\right\rangle$ o valor médio de $f\left(x, p_{x}\right)$ ao longo do intervalo de integração.

Como $\Delta_{1} x \rightarrow 0$ na travessia da n-ésima função delta, $x$ permanece constante e igual a $x_{n}$ nesse intervalo de tempo e, portanto, tem-se

$$
\Delta_{1} p_{x} \cong \frac{\varepsilon k}{2} \operatorname{sen}\left(k x_{n}\right) \int_{n T-0}^{n T+0} \delta(t-n T) \mathrm{d} t=\frac{\varepsilon k}{2} \operatorname{sen}\left(k x_{n}\right)
$$

Dessa forma, tem-se que os valores de $x$ e $p_{x}$ imediatamente após a passagem da função delta centrada em $t=n T$ são dados por

$$
\begin{aligned}
& x_{n}^{+}=x_{n}+\Delta_{1} x=x_{n}, \\
& p_{x, n}^{+}=p_{x, n}+\Delta_{1} p_{x}=p_{x, n}+\frac{\varepsilon k}{2} \operatorname{sen}\left(k x_{n}\right) .
\end{aligned}
$$

Utilizando a transformação canônica entre as variáveis $\left(x, p_{x}\right)$ e $(I, \theta)$, também são obtidos os valores de $I$ e $\theta$ imediatamente após a função delta centrada em $t=n T$

$$
\begin{aligned}
& I_{n}^{+}=\frac{1}{2}\left[\left(x_{n}^{+}\right)^{2}+\left(p_{x, n}^{+}\right)^{2}\right]=\frac{1}{2}\left\{x_{n}^{2}+\left[p_{x, n}+\frac{\varepsilon k}{2} \operatorname{sen}\left(k x_{n}\right)\right]^{2}\right\}, \\
& I_{n}^{+}=\frac{1}{2}\left\{2 I_{n} \operatorname{sen}^{2} \theta_{n}+\left[\sqrt{2 I_{n}} \cos \theta_{n}+\frac{\varepsilon k}{2} \operatorname{sen}\left(k \sqrt{2 I_{n}} \operatorname{sen} \theta_{n}\right)\right]^{2}\right\}, \\
& \theta_{n}^{+}=\operatorname{arctg}\left(\frac{x_{n}^{+}}{p_{x, n}^{+}}\right)=\operatorname{arctg}\left[\frac{2 x_{n}}{2 p_{x, n}+\varepsilon k \operatorname{sen}\left(k x_{n}\right)}\right], \\
& \theta_{n}^{+}=\operatorname{arctg}\left[\frac{2 \sqrt{2 I_{n}} \operatorname{sen} \theta_{n}}{2 \sqrt{2 I_{n}} \cos \theta_{n}+\varepsilon k \operatorname{sen}\left(k \sqrt{2 I_{n}} \operatorname{sen} \theta_{n}\right)}\right] .
\end{aligned}
$$

Entre dois pulsos de onda consecutivos, o termo $\varepsilon V$ correspondente à onda 
eletrostática se anula e as Hamiltonianas (6) e (7) tornam-se integráveis e independentes do tempo. Contudo, o primeiro termo da Hamiltoniana (6) depende das variáveis $x$ e $p_{x}$, enquanto o primeiro termo da Hamiltoniana (7) é função apenas da variável de ação $I$. Essa propriedade da Hamiltoniana (7) permite o cálculo exato das variações sofridas pelas grandezas $I$ e $\theta$ entre a direita da n-ésima função delta e a esquerda da função delta centrada em $t=(n+1) T$,

$$
\begin{gathered}
\Delta_{2} I=\left.\int_{n T}^{(n+1) T} \frac{\mathrm{d} I}{\mathrm{~d} t}\right|_{H=H_{0}} \mathrm{~d} t=-\int_{n T}^{(n+1) T} \frac{\partial H_{0}}{\partial \theta} \mathrm{d} t=0 \\
\Delta_{2} \theta=\left.\int_{n T}^{(n+1) T} \frac{\mathrm{d} \theta}{\mathrm{d} t}\right|_{H=H_{0}} \mathrm{~d} t=\int_{n T}^{(n+1) T} \frac{\partial H_{0}}{\partial I} \mathrm{~d} t=\int_{n T}^{(n+1) T} \frac{1}{\sqrt{1+2 I}} \mathrm{~d} t
\end{gathered}
$$

Como $I$ não sofre alterações entre as funções delta centradas em $t=n T$ e $t=(n+1) T, I$ é constante e igual a $I_{n}^{+}$nesse intervalo de tempo. Portanto, a variação sofrida pela variável $\theta$ é dada por

$$
\Delta_{2} \theta=\frac{1}{\sqrt{1+2 I_{n}^{+}}} \int_{n T}^{(n+1) T} \mathrm{~d} t=\frac{T}{\sqrt{1+2 I_{n}^{+}}} .
$$

Dessa forma, os valores de $I$ e $\theta$ imediatamente antes da função delta centrada em $t=(n+1) T$ são dados por

$$
\begin{aligned}
& I_{n+1}=I_{n}^{+}+\Delta_{2} I=I_{n}^{+}, \\
& \theta_{n+1}=\theta_{n}^{+}+\Delta_{2} \theta,
\end{aligned}
$$

e a partir dessas expressões obtém-se o mapa (8) que descreve a evolução temporal do sistema nas variáveis $I$ e $\theta[16,17]$

$$
\begin{aligned}
& I_{n+1}=\frac{1}{2}\left\{2 I_{n} \operatorname{sen}^{2} \theta_{n}+\left[\sqrt{2 I_{n}} \cos \theta_{n}+\frac{1}{2} \varepsilon k \operatorname{sen}\left(k \sqrt{2 I_{n}} \operatorname{sen} \theta_{n}\right)\right]^{2}\right\}, \\
& \theta_{n+1}=\operatorname{arctg}\left[\frac{2 \sqrt{2 I_{n}} \operatorname{sen} \theta_{n}}{2 \sqrt{2 I_{n}} \cos \theta_{n}+\varepsilon k \operatorname{sen}\left(k \sqrt{2 I_{n}} \operatorname{sen} \theta_{n}\right)}\right]+\frac{T}{\sqrt{1+2 I_{n+1}}}(\bmod 2 \pi) .
\end{aligned}
$$

O determinante jacobiano 


$$
|J|=\left|\begin{array}{cc}
\frac{\partial I_{n+1}}{\partial I_{n}} & \frac{\partial I_{n+1}}{\partial \theta_{n}} \\
\frac{\partial \theta_{n+1}}{\partial I_{n}} & \frac{\partial \theta_{n+1}}{\partial \theta_{n}}
\end{array}\right|
$$

do mapa (8) é unitário e por esse motivo, o mapa é dito simplético. Isso significa que o mapa (8) é conservativo, preservando a área do espaço de fases e a energia total do sistema [14, 40]. Além disso, o mapa obtido nesta seção é exato e possui a importante característica de ser totalmente explícito (o termo $I_{n+1}$ na expressão para $\theta_{n+1}$ pode ser escrito explicitamente em função de $I_{n}$ e $\theta_{n}$ utilizando-se a primeira equação do mapa).

Observa-se ainda que o mapa (8) poderia ter sido escrito com o mesmo grau de complexidade nas variáveis $\left(x, p_{x}\right)$, entretanto o uso de variáveis de ângulo-ação é mais conveniente uma vez que a ação é conservada na ausência de perturbação. Isso pode ser visto facilmente na Figura 2 que mostra o espaço de fases ${ }^{4}$ do sistema para $\varepsilon=0$.

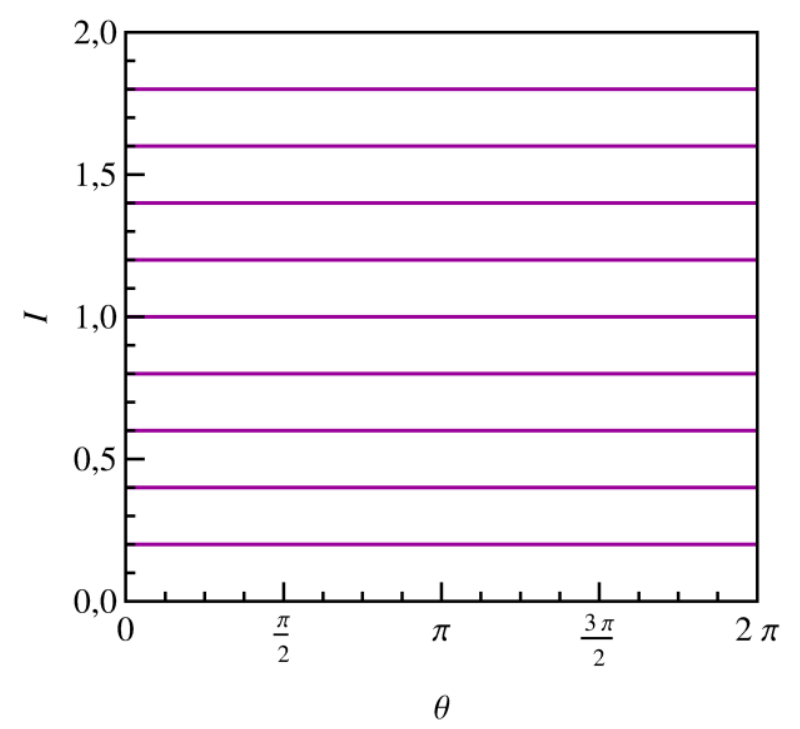

Figura 2: Espaço de fases do sistema para $\varepsilon=0$.

O mapa padrão clássico mencionado na Introdução desta tese é dado por [14, 40]

$$
\begin{aligned}
& I_{n+1}=I_{n}+\varepsilon \operatorname{sen} \theta_{n}, \\
& \theta_{n+1}=\theta_{n}+I_{n+1} .
\end{aligned}
$$

\footnotetext{
${ }^{4}$ Nesta tese, os espaços de fases analisados correspondem a mapas estroboscópicos que apresentam os valores de $I$ e $\theta$ apenas nos instantes de tempo imediatamente anteriores aos pulsos de onda centrados em $t=n T$.
} 
Diferente do que ocorre com o mapa padrão clássico, o mapa (8) apresenta uma forte não linearidade em sua dependência com a amplitude $\varepsilon$ da onda eletrostática. Isso se deve à presença do campo magnético $\vec{B}=B_{0} \hat{z}$ que altera a energia cinética da partícula. Na presença de um campo magnético, a energia cinética é função do momento e da posição da partícula, como mostra a Hamiltoniana (6). Para essa Hamiltoniana, ambas as variáveis $x$ e $p_{x}$ sofrem alterações entre dois pulsos de onda consecutivos, o que torna o mapa (8) não linear com relação à amplitude $\varepsilon$ da onda eletrostática.

Por outro lado, o mapa padrão descreve a interação entre uma partícula e uma onda eletrostática na ausência de campos magnéticos externos. Nesse caso, cada termo da Hamiltoniana só depende de uma das variáveis do sistema. O termo correspondente à energia cinética da partícula é função apenas do momento. Enquanto o termo de energia potencial associado à onda eletrostática só depende da coordenada $x$. Isso faz com que uma das variáveis permaneça constante no intervalo de duração de um pulso de onda, enquanto a outra variável não varia entre dois pulsos de onda consecutivos. Essa característica do sistema simplifica os cálculos e como resultado o mapa padrão é linear em $\varepsilon$.

Logo, o mapa (8) pode ser interpretado como uma versão relativística e magnetizada do mapa padrão clássico. Entretanto, apesar de estarem relacionados, os dois mapas apresentam estruturas bastante diferentes. Duas dessas diferenças serão discutidas no Capítulo 4 e se referem à alteração na posição dos pontos periódicos e às mudanças que ocorrem no processo de aceleração regular da partícula devido à não linearidade do mapa (8) com relação a $\varepsilon$ [16].

Embora o mapa exato (8) seja não linear em $\varepsilon$, é possível obter uma versão linearizada, válida somente para $\varepsilon \ll 1$. Essa versão linearizada é obtida quando se utiliza apenas a Hamiltoniana (7) escrita como função das variáveis $(I, \theta)$. Considerando que na travessia da função delta centrada em $t=n T, I \rightarrow I_{n+1}$ e $\theta \rightarrow \theta_{n}$, o mapa linearizado em $\varepsilon$ pode ser escrito em uma forma canônica dada por

$$
\begin{aligned}
& I_{n+1}=I_{n}+\frac{1}{2} \varepsilon k \sqrt{2 I_{n+1}} \cos \theta_{n} \operatorname{sen}\left(k \sqrt{2 I_{n+1}} \operatorname{sen} \theta_{n}\right), \\
& \theta_{n+1}=\theta_{n}-\frac{\varepsilon k \operatorname{sen} \theta_{n}}{2 \sqrt{2 I_{n+1}}} \operatorname{sen}\left(k \sqrt{2 I_{n+1}} \operatorname{sen} \theta_{n}\right)+\frac{T}{\sqrt{1+2 I_{n+1}}}(\bmod 2 \pi) .
\end{aligned}
$$

A consideração de que $I \rightarrow I_{n+1}$ e $\theta \rightarrow \theta_{n}$ na travessia da função delta é feita para garantir 
que o mapa linearizado preserve o caráter simplético das equações de Hamilton. Dessa forma, o mapa (9) conserva a área no espaço de fases e a energia total do sistema. Se o mapa (8) fosse linearizado em $\varepsilon$ diretamente, sem a consideração de que $I \rightarrow I_{n+1}$ e $\theta \rightarrow \theta_{n}$ na travessia da função delta, o mapa obtido não seria simplético e não preservaria a área no espaço de fases, nem a energia total do sistema.

Além disso, é importante ressaltar que a Hamiltoniana (7) e o mapa (8) não incluem nenhum efeito relativo à emissão de radiação por uma partícula carregada e acelerada. Tipicamente, aceleradores de partículas apresentam campos magnéticos da ordem de 1 Tesla. Por exemplo, considerando um elétron que se move com velocidade $v_{\perp} \cong 0,9999 c$ perpendicular ao campo magnético, a energia emitida pelo elétron em um período ciclotrônico é aproximadamente $4,87 \times 10^{-10} K[50,55,56]$, onde $K$ denota a energia cinética do elétron. Por outro lado, a energia máxima emitida pelo elétron devido a um pulso de onda é estimada como $2,21 \times 10^{-8} K[50,55,56]$, para um pulso ultracurto com duração na ordem de $10^{-16}$ segundos [57], e parâmetros adimensionais $k=10$ e $\varepsilon=1$. Para valores mais elevados de $k$ e $\varepsilon$, a dinâmica do sistema se torna extremamente caótica. A partir dessas estimativas, verifica-se que mesmo para velocidades próximas à velocidade da luz, a energia emitida pelo elétron é muito menor do que sua energia cinética, e o processo de emissão de radiação, para os propósitos desta tese, pode ser desconsiderado.

\subsection{ESPAÇO DE FASES E TRANSIÇÃO PARA O CAOS}

Como visto na Figura 2, o espaço de fases nas variáveis de ângulo-ação $I \times \theta$ apresenta apenas curvas estendidas na ausência de perturbação. Para $\varepsilon \neq 0$, o espaço de fases passa a apresentar curvas fechadas, conhecidas como ilhas de ressonância. Nas ilhas de ressonância, a razão entre a frequência de rotação da partícula no plano $x \mathrm{O} y$ e a frequência da onda eletrostática é um número racional [14, 40, 58-63]. Essa razão entre as frequências do sistema é chamada de número de rotação e representada pela letra $\Omega$. Logo, para $\Omega=s / r$, com $r$ e $s$ dois números inteiros, positivos, não nulos e primos entre si, observa-se no espaço de fases a formação de ilhas da ressonância $(r, s)$ na posição $I_{r, s}$. As ressonâncias primárias do sistema são discutidas com mais detalhes no Capítulo 3. 
A ressonância principal do sistema é aquela para a qual cada ciclo da onda eletrostática corresponde a um giro completo da partícula, que se move sob a ação do campo magnético, no plano $x \mathrm{O} y$ [16]. Isso significa que a frequência da onda é igual à frequência do movimento não perturbado. Para a ressonância principal, a razão entre essas frequências é $\Omega=1 / 1$ e entre dois pulsos de onda consecutivos a partícula descreve um ângulo $\Delta \theta=$ $\theta_{n+1}-\theta_{n}=2 \pi$ no plano $x \mathrm{O} y$, de tal forma que $I_{n+1} \cong I_{n} \equiv I_{1,1}$. Substituindo esses resultados na primeira equação do mapa (8), tem-se

$$
I_{1,1}=I_{1,1}+\frac{\varepsilon^{2} k^{2}}{8} \operatorname{sen}^{2}\left(k \sqrt{2 I_{1,1}} \operatorname{sen} \theta_{n}\right)+\frac{\varepsilon k}{2} \sqrt{2 I_{1,1}} \cos \theta_{n} \operatorname{sen}\left(k \sqrt{2 I_{1,1}} \operatorname{sen} \theta_{n}\right)
$$

de onde segue que

$$
\operatorname{sen}\left(k \sqrt{2 I_{1,1}} \operatorname{sen} \theta_{n}\right)=0 \quad \text { ou } \quad \frac{\varepsilon k}{4} \operatorname{sen}\left(k \sqrt{2 I_{1,1}} \operatorname{sen} \theta_{n}\right)+\sqrt{2 I_{1,1}} \cos \theta_{n}=0,
$$

pois $\varepsilon \neq 0$ e $k \neq 0$. Utilizando a primeira solução (10) na segunda equação do mapa (8), obtém-se o valor de $I_{1,1}$ para a ressonância principal do sistema

$$
I_{1,1} \cong \frac{T^{2}-4 \pi^{2}}{8 \pi^{2}}
$$

Como a ação é uma grandeza positiva por definição, a expressão (11) indica que a ressonância principal só está presente no espaço de fases do sistema para $T \geq 2 \pi$. Lembrando que a frequência adimensional $\omega$ da onda eletrostática é medida em unidades da frequência ciclotrônica $\omega_{c}=q B_{0} / m$, tem-se que $\omega=1$ e, portanto, $T=2 \pi / \omega=2 \pi$ quando a frequência da onda eletrostática coincide com a frequência ciclotrônica. Logo, para que a ressonância principal esteja presente no espaço de fases, é preciso que o período da onda eletrostática seja maior do que o período ciclotrônico. Além disso, quanto maior o valor do período $T$, mais a ressonância principal é deslocada para valores elevados da ação $I$.

A partir do mapa (8) obtêm-se os valores de $I$ e $\theta$ em qualquer instante de tempo desde que sejam conhecidos seus valores no instante inicial. Dessa forma, é possível construir várias simulações numéricas para diferentes valores dos parâmetros de controle do sistema. Entretanto, ao longo desta tese serão considerados em geral casos em que a onda eletrostática possui um período próximo ao período ciclotrônico, uma vez que diversos sistemas possuem essa característica (como, por exemplo, plasmas aquecidos em tokamaks, assim como outros sistemas discutidos nas Refs. [45, 46]). 
A Figura 3 mostra o espaço de fases do sistema construído a partir do mapa (8) para $T=2 \pi(1+1 / 15), k=2$ e diversos valores da amplitude de perturbação $\varepsilon$. Para geração dessa figura foi escolhido o período $T=2 \pi(1+1 / 15)$, que é ligeiramente maior do que o período ciclotrônico $T_{c}=2 \pi$, para que a ressonância principal esteja presente no espaço de fases do sistema de acordo com a condição (11). Além disso, considera-se que $k=2$ é um número de onda representativo dos modos que serão analisados ao longo dessa tese.

O espaço de fases do sistema é construído iterando-se algumas condições iniciais e todas as iterações são plotadas em um gráfico $I \times \theta$. As condições iniciais são escolhidas de tal forma que seja possível visualizar as principais estruturas do sistema. Isso inclui as ilhas de ressonância, as curvas invariantes, as regiões de comportamento caótico e como esses diferentes tipos de trajetória se intercalam e estão distribuídos no espaço de fases. Sendo assim, o espaço de fases fornece uma visão geral do comportamento do sistema e a partir dele é possível visualizar quais regiões e, portanto, quais condições iniciais fornecem o comportamento desejado para o sistema.

Para facilitar a identificação dos diferentes tipos de trajetória, todos os espaços de fases desta tese foram construídos utilizando o mesmo esquema de cores. Nas figuras, as curvas invariantes são representadas pela cor roxa e as trajetórias caóticas são representadas em azul. As sepatrizes das ilhas de ressonância foram construídas em cinza. Já as ilhas de ressonância estão representadas em verde, vermelho, amarelo e ciano. No caso das ressonâncias, foram utilizadas cores diferentes para distinguir as ilhas de cadeias independentes, mas que pertencem a uma mesma ressonância. Isto é, uma condição inicial verde da ressonância $(r, s)$ só gera as ilhas representadas em verde. Uma condição inicial vermelha gera apenas as ilhas representadas em vermelho da ressonância $(r, s)$, e assim sucessivamente. Lembrando que cada ressonância $(r, s)$ está localizada em uma posição diferente com relação à variável de ação $I$ e uma condição inicial que pertence a uma dada ressonância $(r, s)$ só pode gerar ilhas dessa ressonância.

Para $\varepsilon=0$ como na Figura 2, o sistema é integrável, ou seja, $H=H_{0}(I)$ e a variável de ação $I$ é conservada. Nessa situação, o espaço de fases apresenta apenas curvas estendidas chamadas de curvas ou toros invariantes. Quando $\varepsilon \neq 0$, a perturbação provocada pela onda eletrostática altera a estrutura do espaço de fases. Mesmo para $\varepsilon=2,5 \times 10^{-4}$ como na Figura 3(a), é possível ver a formação de ilhas de ressonância nas posições $I_{1,1} \cong 0,069$ e $I_{2,1} \cong 1,776$. Entretanto, como o valor de $\varepsilon$ é muito baixo nessa figura, a largura das ilhas de 
(a) $\varepsilon=2,5 \times 10^{-4}$

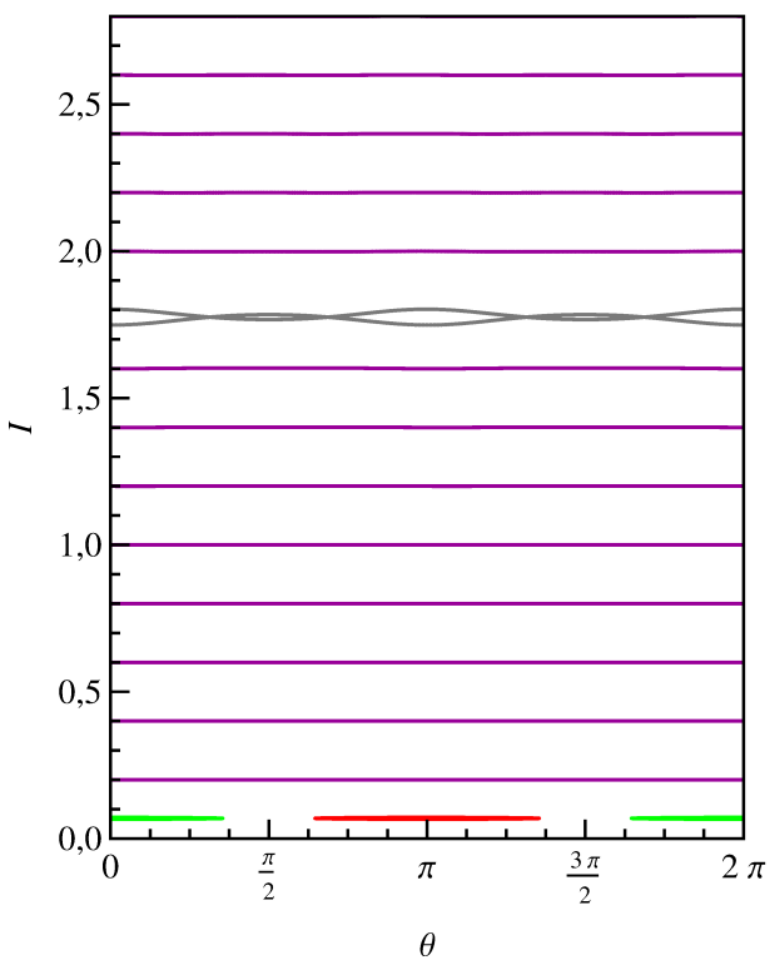

(c) $\varepsilon=0,125$

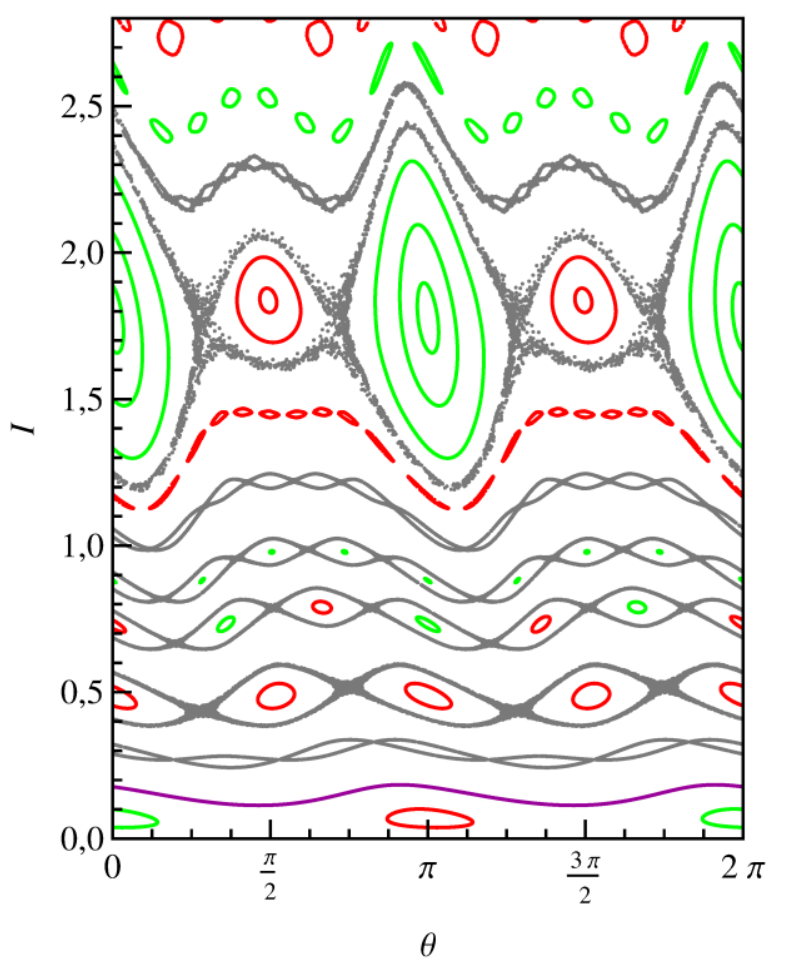

(b) $\varepsilon=2,5 \times 10^{-2}$

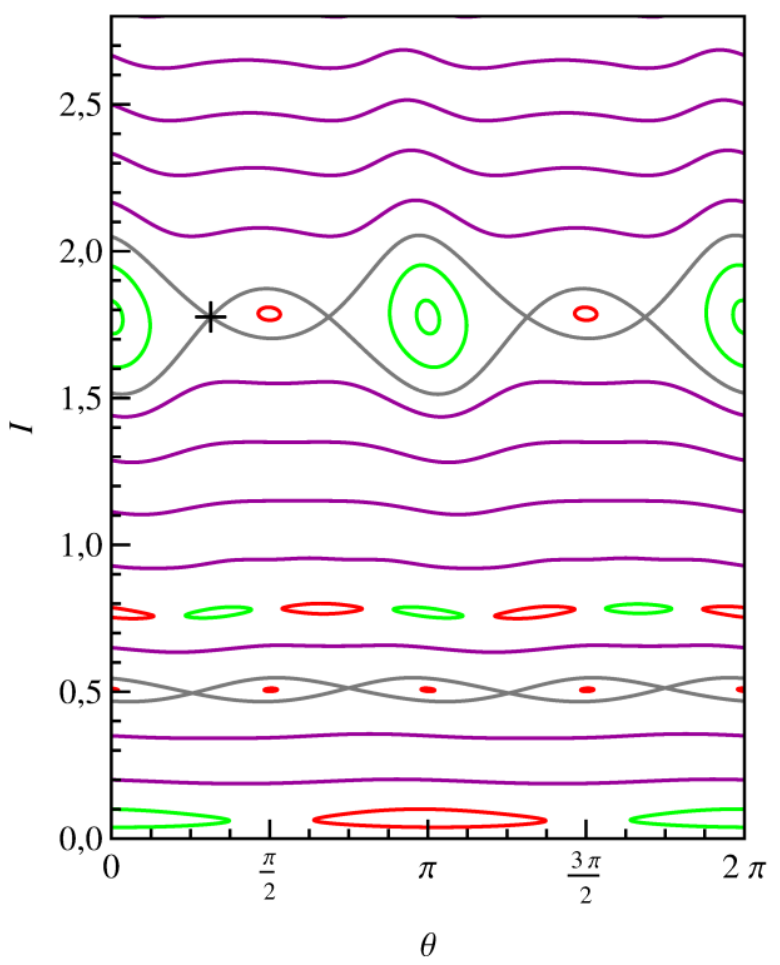

(d) $\varepsilon=0,200$

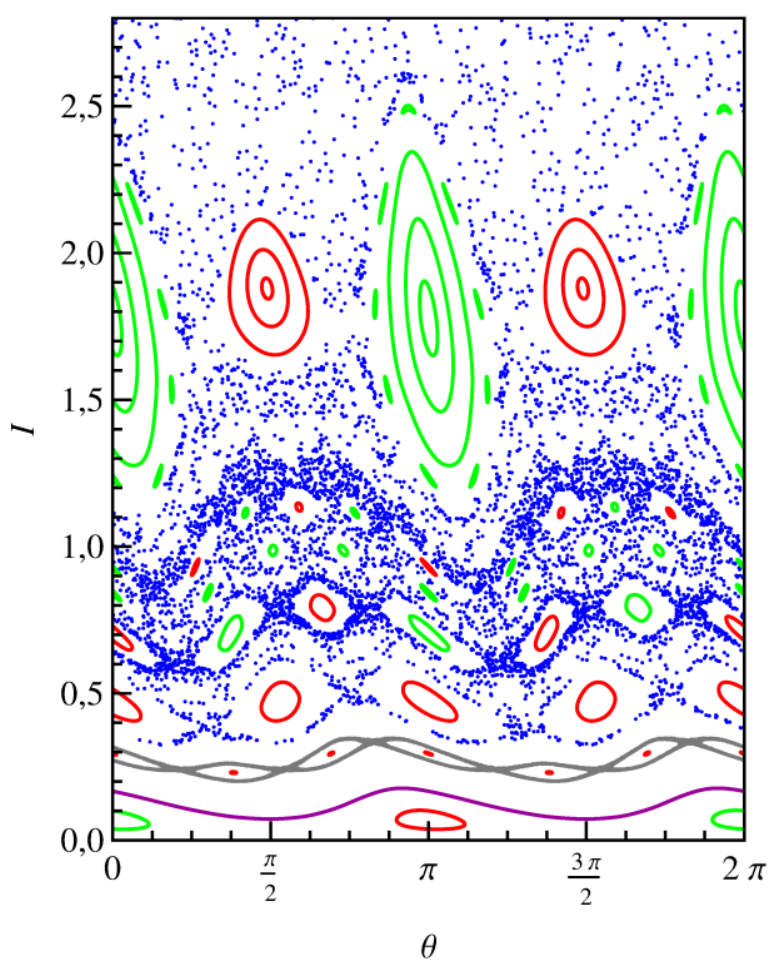

Figura 3: Espaço de fases do sistema para $T=2 \pi(1+1 / 15), k=2$ e a) $\varepsilon=2,5 \times 10^{-4}$; b) $\varepsilon=2,5 \times 10^{-2}$; c) $\varepsilon=0,125$; d) $\varepsilon=0,200$; e) $\varepsilon=0,250$; f) $\varepsilon=0,375$. 
(e) $\varepsilon=0,250$

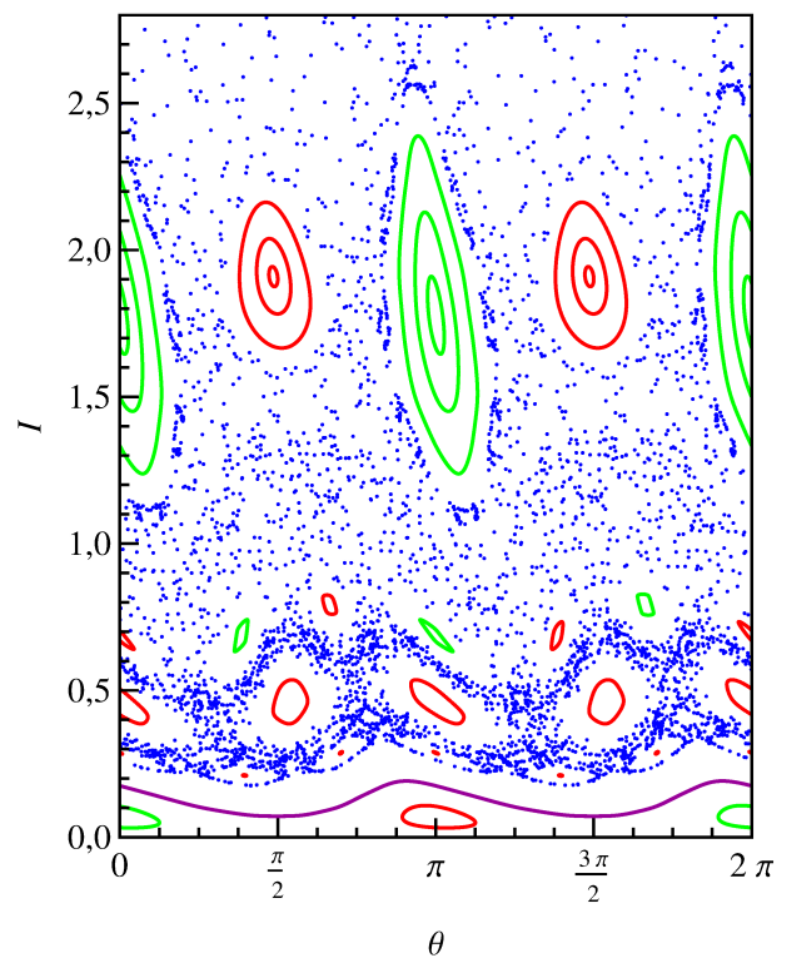

(f) $\varepsilon=0,375$

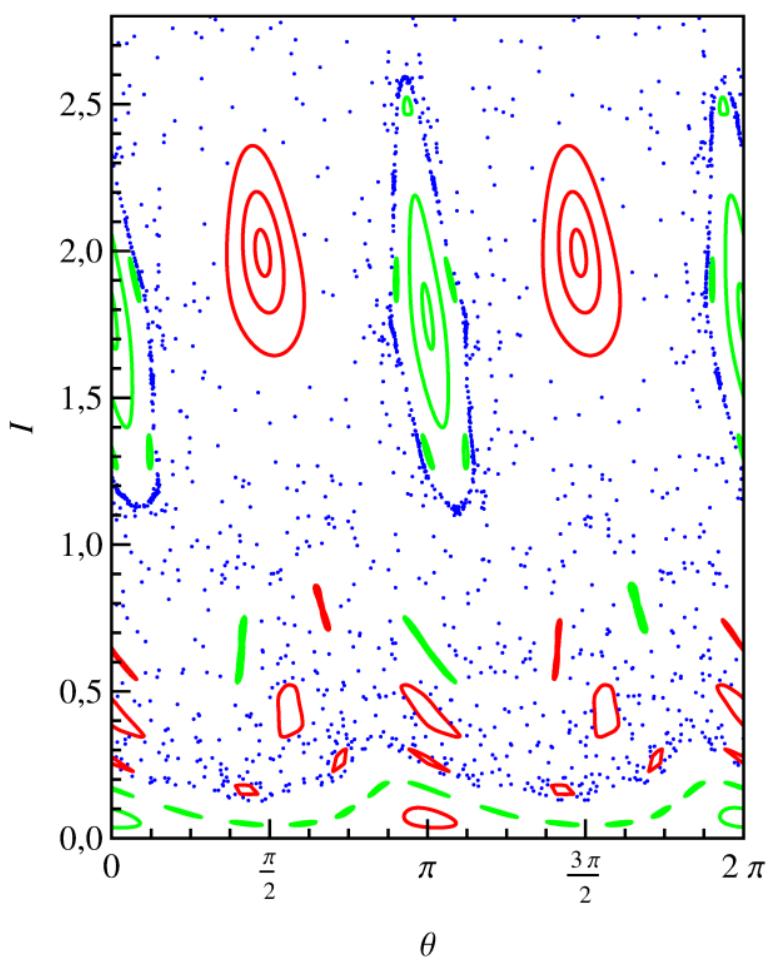

Figura 3: Continuação.

ressonância também é reduzida e a estrutura do espaço de fases é praticamente a mesma da Figura 2, isto é, a Figura 3(a) também é preenchida em quase toda sua extensão por toros invariantes. A resolução utilizada para construção da Figura 3(a) não permite a visualização de detalhes das ilhas localizadas em $I_{1,1} \cong 0,069$ e $I_{2,1} \cong 1,776$, assim como não é possível visualizar as inúmeras ilhas de ressonâncias primárias localizadas nas posições $I_{r, s}$. Embora essas ressonâncias estejam presentes no espaço de fases, elas são extremamente pequenas para a resolução utilizada na Figura 3(a).

A Figura 4 mostra uma ampliação da Figura 3(a) nas regiões das ressonâncias $(1,1)$ e $(2,1)$. Nessa figura, é possível ver com mais detalhes a estrutura das ilhas de ressonância do sistema e as distorções que elas provocam nos toros invariantes ao seu redor. Na Figura 4(a), por exemplo, observa-se os pontos periódicos ${ }^{5}$ da ressonância $(1,1)$, que é a ressonância principal do sistema. Os pontos elípticos ${ }^{6}$ estão localizados em $\theta \cong 0 ; \pi$ e ao redor deles são formadas as ilhas de ressonância. Os pontos hiperbólicos por sua vez estão localizados em $\theta \cong \pi / 2 ; 3 \pi / 2$ e eles separam as ilhas da ressonância $(1,1)$. No interior da

\footnotetext{
5 Pontos periódicos são pontos que se repetem após um número determinado de iterações do mapa.

6 Pontos elípticos (hiperbólicos) são pontos periódicos centralmente estáveis (instáveis) do mapa $I_{n+1}=I_{n+1}\left(I_{n}, \theta_{n}\right) ; \theta_{n+1}=\theta_{n+1}\left(I_{n}, \theta_{n}\right)$.
} 
separatriz, representada em cinza, as trajetórias são curvas fechadas no espaço de fases e centradas ao redor dos pontos elípticos. Na região exterior à separatriz, as trajetórias são curvas invariantes e abertas no espaço de fases.

Aumentando o valor de $\varepsilon$ como na Figura 3(b), o espaço de fases ainda apresenta inúmeros toros invariantes. Entretanto, esses toros não são mais linhas retas como na Figura 2, pois eles são deformados pelas ressonâncias. Para $\varepsilon=2,5 \times 10^{-2}$, a largura das ilhas de ressonância aumenta e elas se tornam mais evidentes no espaço de fases. Na Figura 3(b), é possível visualizar com clareza as ressonâncias $(1,1),(4,3),(3,2)$ e $(2,1)$, localizadas respectivamente em $I_{1,1} \cong 0,069, I_{4,3} \cong 0,511, I_{3,2} \cong 0,780$ e $I_{2,1} \cong 1,776$. Em geral, observase que as maiores ilhas no espaço de fases são aquelas para as quais $s=1$.

(a) Ressonância $(1,1)$

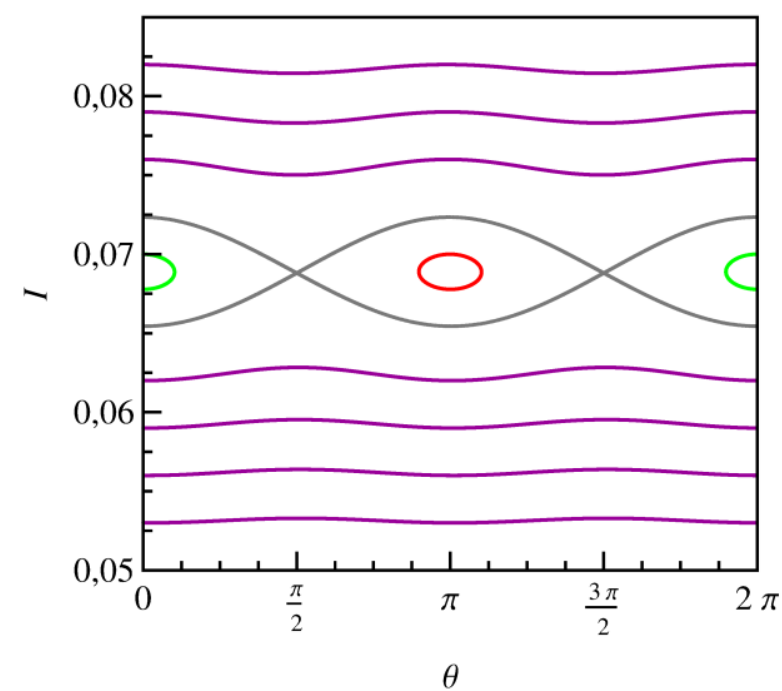

(b) Ressonância $(2,1)$

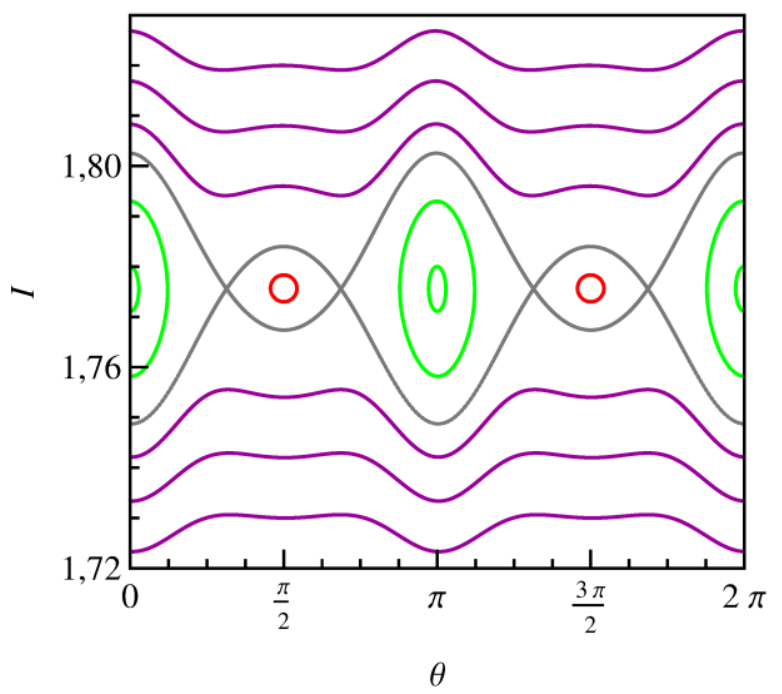

Figura 4: Ampliação das ilhas das ressonâncias a) $(1,1)$ e b) $(2,1)$ identificadas na Figura 3(a).

Para algumas ressonâncias $(r, s)$, o sistema apresenta apenas uma cadeia com $r$ ilhas. Esse é o caso, por exemplo, da ressonância $(4,3)$ representada pelas trajetórias em vermelho na posição $I_{4,3} \cong 0,511$ da Figura $3(\mathrm{~b})$. Os pontos elípticos centrais de cada ilha correspondem a uma única órbita periódica. A trajetória se move de um ponto periódico para o outro e entre dois pulsos de onda consecutivos, tem-se $\Delta \theta=\theta_{n+1}-\theta_{n}=2 \pi s / r=3 \pi / 2$ $(\bmod 2 \pi)$. Dessa forma, após 4 iterações do mapa (8) o ponto elíptico inicial se repete. Algo semelhante ocorre com as órbitas centradas ao redor dos pontos elípticos da ressonância $(4,3)$. As trajetórias se movem de uma ilha para a outra, de tal forma que uma única trajetória forma as quatro ilhas da cadeia, cada ilha centrada ao redor de um ponto elíptico. Entretanto, para 
essas órbitas o número de rotação $\Omega$ é irracional e os pontos nunca se repetem. Por esse motivo, uma única trajetória preenche densamente as quatro ilhas da cadeia no espaço de fases.

Por outro lado, algumas ressonâncias $(r, s)$ apresentam não uma, mas duas cadeias com $r$ ilhas cada. Isso acontece com as ressonâncias $(1,1),(2,1)$ e $(3,2)$ identificadas na Figura 3(b). Nessa figura, as cadeias de uma mesma ressonância estão distinguidas pelas cores verde e vermelho. Por exemplo, a ressonância $(2,1)$ localizada ao redor de $I_{2,1} \cong 1,776$ apresenta duas cadeias com duas ilhas cada. Os pontos elípticos centrais em $\theta \cong 0$; $\pi$ e $\theta \cong \pi / 2 ; 3 \pi / 2$ formam duas órbitas periódicas distintas. Em cada cadeia, a trajetória da partícula se move de um ponto elíptico para o outro repetidamente, de tal forma que entre dois pulsos de onda consecutivos tem-se $\Delta \theta=\theta_{n+1}-\theta_{n}=2 \pi s / r=\pi \quad(\bmod 2 \pi)$. As ressonâncias primárias e o número de cadeias de ilhas que elas apresentam são discutidos em detalhes no Capítulo 3.

Na resolução utilizada para construção da Figura 3(b), todas as trajetórias do sistema parecem regulares. Entretanto, ampliando a região próxima a um ponto hiperbólico como na Figura 5, observa-se que mesmo para valores muito baixos de $\varepsilon$ o sistema possui trajetórias caóticas. Na verdade, as trajetórias nas vizinhanças dos pontos hiperbólicos sempre são caóticas, por menor que seja o valor de $\varepsilon$ desde que $\varepsilon \neq 0[14,40]$. Isso ocorre devido à grande complexidade da dinâmica ao redor dos pontos hiperbólicos. Lichtenberg e Lieberman apresentam na página 185 da Ref. [14] uma explicação bastante detalhada sobre o comportamento das trajetórias nas vizinhanças de pontos hiperbólicos e como a complexidade desse comportamento dá origem ao caos.

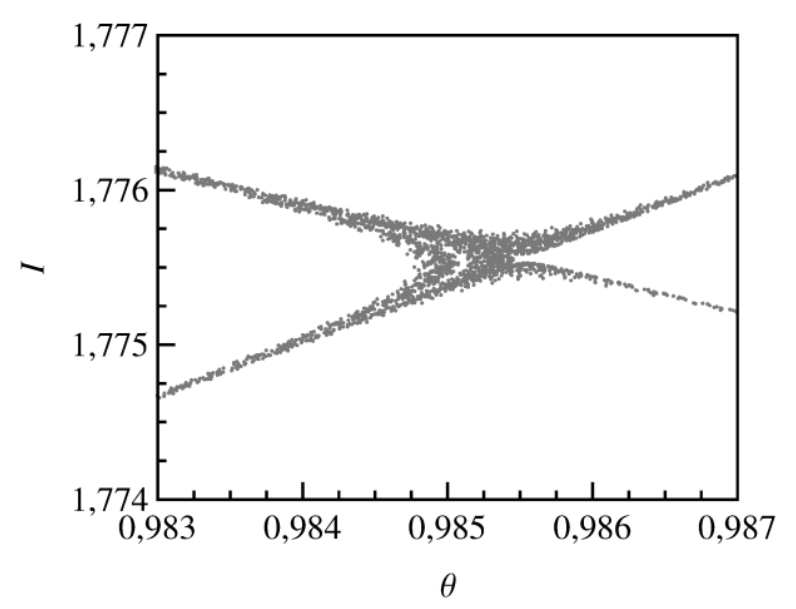

Figura 5: Ampliação da região próxima ao ponto hiperbólico indicado por uma cruz preta na Figura 3(b). 
Para $\varepsilon=0,125$ como na Figura 3(c), as ilhas de ressonância aumentam consideravelmente de tamanho, deformando ainda mais os toros invariantes. Além disso, o aumento da largura das ressonâncias faz com que seja possível visualizar cada vez mais cadeias de ilhas no espaço de fases do sistema. Observa-se ainda que além dos toros invariantes, as cadeias de ilhas menores também são deformadas pelas ilhas maiores do sistema (ressonâncias $(1,1)$ e $(2,1)$ ). Isso faz com que os pontos elípticos e hiperbólicos das cadeias de ilhas menores sejam deslocados com relação às variáveis $I$ e $\theta$. Esse deslocamento é evidente se compararmos, por exemplo, a posição dos pontos periódicos das ressonâncias $(3,2)$ e $(4,3)$ nas Figuras 3(b) e 3(c). Na Figura 3(c), essas cadeias de ilhas sofrem uma ondulação devido, principalmente, ao aumento da largura da ressonância $(2,1)$.

$\mathrm{Na}$ Figura 3(b), o caos estava localizado apenas ao redor dos pontos hiperbólicos e não era possível visualizá-lo na resolução utilizada. Já na Figura 3(c), as trajetórias caóticas se tornam mais evidentes, pois elas não estão mais localizadas apenas nas vizinhanças dos pontos hiperbólicos. Nessa figura, o caos pode ser visto com nitidez nas separatrizes das ilhas de ressonância e a partir das separatrizes, ele começa a se espalhar pelo espaço de fases. Entretanto, para $\varepsilon=0,125$ ainda existem diversos toros invariantes separando as ressonâncias do sistema. Esses toros invariantes atuam como barreiras, confinando as trajetórias caóticas nas regiões próximas às separatrizes das ilhas e impedindo que o caos se espalhe por todo o espaço de fases. A Figura 6 mostra uma ampliação da região $0,2 \leq I \leq 0,9$ para os mesmos parâmetros utilizados na Figura 3(c). Na Figura 6, observa-se com mais clareza a separatriz caótica das ressonâncias do sistema e os toros invariantes que separam as cadeias de ilhas e confinam as trajetórias caóticas.

Como visto na transição da Figura 3(a) para 3(c), quanto maior a amplitude da perturbação, maiores são as ilhas de ressonância do sistema. Para valores suficientemente elevados de $\varepsilon$, as ilhas de duas ressonâncias próximas crescem até se sobreporem, destruindo todos os toros invariantes que as separavam. Com a destruição dos toros invariantes, as trajetórias caóticas podem se mover livremente em toda a região ao redor das duas ressonâncias que se sobrepuseram. Na Figura 3(d), construída para $\varepsilon=0,200$, grande parte dos toros invariantes do sistema já foram destruídos e as trajetórias caóticas preenchem quase todo o espaço de fases, com exceção das ilhas de ressonância e da região $I<0,2$ que ainda possui toros invariantes. Essa situação é conhecida como caos global, pois não existem mais toros invariantes limitando as trajetórias caóticas na região $I>0,3$. Também é dito que as trajetórias caóticas na região $I>0,3$ formam um mar de caos. 


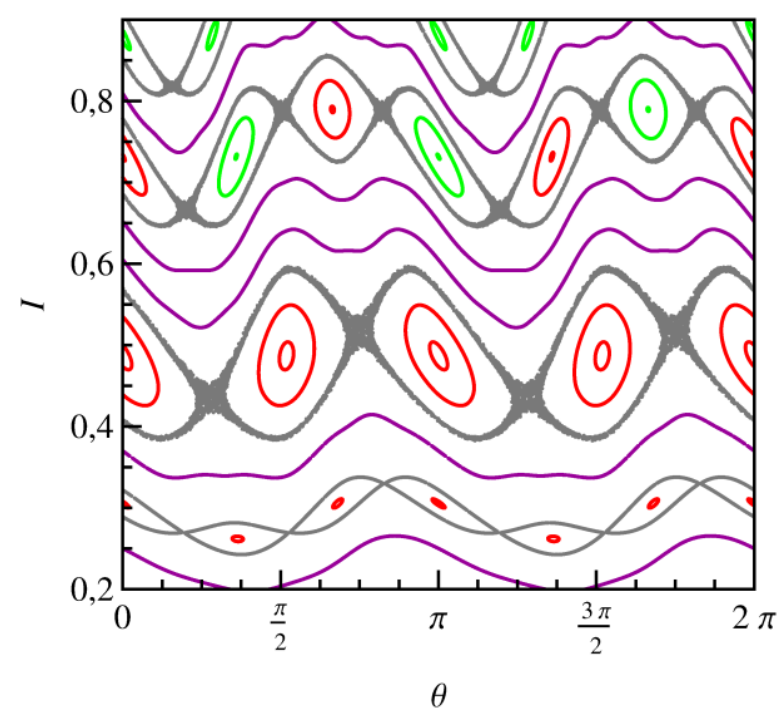

Figura 6: Ampliação de algumas cadeias de ilhas da Figura 3(c).

Na Figura 3(d), observa-se o surgimento de ressonâncias de ordens mais elevadas imersas no mar de caos. Essas ressonâncias não podem ser estimadas a partir de expansões de primeira ordem da Hamiltoniana (7) e do mapa (8), e elas são formadas pelo acoplamento de ressonâncias primárias que ocorre quando a amplitude da perturbação aplicada ao sistema torna-se elevada. Além disso, ressonâncias de ordens mais elevadas também são formadas ao redor ou no interior das cadeias de ilhas das ressonâncias primárias. Ao redor das ilhas centradas em $I_{2,1} \cong 1,776$ e $\theta \cong 0 ; \pi$, por exemplo, surge uma cadeia secundária formada por nove ilhas menores.

Também é importante ressaltar que o comportamento da partícula não é uniforme em toda a região caótica do espaço de fases. Observa-se que as trajetórias caóticas são mais densas ao redor das cadeias de ilhas presentes na região $0,3<I<1,3$. Isso significa que uma partícula com condição inicial próxima a uma dessas ilhas fica presa ao redor da cadeia de ilhas correspondente durante um grande número de iterações. Apesar de a trajetória ser caótica, ela demora muitas iterações para conseguir escapar e se afastar da cadeia de ilhas. Além disso, caso a trajetória caótica volte para a região $0,3<I<1,3$, ela ficará presa novamente ao redor das cadeias de ilhas por um grande número de iterações. Essa característica é conhecida como aprisionamento e ela ocorre para valores de $\varepsilon$ ligeiramente maiores do que o valor necessário para a sobreposição de duas ressonâncias. Como dito anteriormente, quando duas ressonâncias se sobrepõem, os toros invariantes que separam as cadeias de ilhas são quebrados. Entretanto, esses toros não são completamente destruídos. Eles são divididos em várias partes separadas por lacunas, formando uma estrutura conhecida 
como conjunto de Cantor e por esse motivo, esses toros passam a ser chamados de cantoros [40].

Os cantoros ainda atuam como barreiras no espaço de fases, confinando as trajetórias caóticas durante algum tempo e formando regiões de aprisionamento. Entretanto, devido às lacunas que existem nos cantoros, as trajetórias caóticas conseguem escapar depois um número suficientemente grande de iterações e a partir de então, elas podem se mover livremente pelo mar de caos. A situação inversa também é possível, ou seja, uma trajetória que se move pelo mar de caos pode entrar em uma região de aprisionamento através das lacunas nos cantoros. Uma vez dentro da região de aprisionamento, a trajetória precisará de um número suficientemente grande de iterações do mapa para conseguir escapar.

Para $I>1,3$ na Figura 3(d), não existem mais cantoros e, portanto, as trajetórias não são aprisionadas nessa região. Isso pode ser visto a partir da densidade de pontos que é bem menor para $I>1,3$ do que na região $0,3<I<1,3$. Logo, uma trajetória caótica preenche quase todo o espaço de fases, podendo assumir valores de ação $I$ arbitrariamente elevados.

Aumentando o valor de $\varepsilon$ como nas Figuras 3(e) e 3(f), os cantoros presentes na região $0,3<I<1,3$ vão sendo destruídos. Até que para valores suficientemente elevados de $\varepsilon$, todos os cantoros são destruídos e não existem mais regiões de aprisionamento no espaço de fases, como pode ser visto na Figura 3(f). Além disso, a densidade de pontos no mar de caos da Figura 3(f) diminui com relação às Figuras 3(d) e 3(e). Isso significa que para valores elevados de $\varepsilon$, a onda eletrostática transfere, em média, mais energia para a partícula e ela atinge valores de ação $I>2,8$ após um número menor de iterações.

Nas Figuras 3(e) e 3(f), também observa-se a destruição das ilhas de ressonâncias primárias imersas no mar de caos. Essas figuras mostram como as trajetórias mais externas das ilhas centradas em $I_{2,1} \cong 1,776$ e $\theta \cong 0 ; \pi$, por exemplo, vão sendo deformadas e destruídas pelo aumento da perturbação. Ao redor dessas ilhas, também é possível ver na Figura 3(f) o surgimento de uma ressonância secundária com cinco ilhas.

Por outro lado, as ilhas da ressonância $(2,1)$ localizadas em $\theta \cong \pi / 2 ; 3 \pi / 2$ continuam a crescer apesar do aumento da perturbação aplicada ao sistema. Essas ilhas só serão destruídas pelo caos para valores muito mais elevados de $\varepsilon$ do que o observado para a destruição das ilhas da ressonância $(2,1)$ centradas em $\theta \cong 0 ; \pi$. Outra característica importante das Figuras 3(e) e 3(f) é o deslocamento dos pontos elípticos da ressonância $(2,1)$ localizados em $\theta \cong \pi / 2 ; 3 \pi / 2$. Enquanto os pontos elípticos dessa ressonância localizados 
em $\theta \cong 0 ; \pi$ permanecem na posição $I_{2,1} \cong 1,776$, os pontos elípticos em $\theta \cong \pi / 2 ; 3 \pi / 2$ são deslocados para valores mais elevados de ação, e quanto maior é a amplitude $\varepsilon$ da perturbação, maior é o valor de $I$ para esses pontos elípticos.

\subsection{CONCLUSÃO}

Neste capítulo, foi analisada a interação de uma partícula relativística com um campo magnético uniforme e uma onda eletrostática e estacionária dada na forma de pulsos periódicos. A partir da Hamiltoniana do sistema, foi obtido um mapa exato e completamente explícito que descreve a evolução temporal do sistema. Esse mapa pode ser considerado como uma versão relativística e magnetizada do mapa padrão clássico. Entretanto, o mapa obtido neste capítulo é não linear com relação à amplitude da onda eletrostática. Por esse motivo, os dois mapas apresentam características distintas, como será discutido no Capítulo 4.

A partir do mapa do sistema, foram construídos alguns espaços de fases para diferentes valores dos parâmetros da onda eletrostática. Isso possibilitou a análise do comportamento do sistema e das alterações provocadas pela presença de ressonâncias não lineares no espaço de fases. Também foi possível analisar como o sistema se torna caótico à medida que a perturbação provocada pela onda aumenta e como as ressonâncias contribuem para o surgimento de trajetórias caóticas. 


\section{RESSONÂNCIAS PRIMÁRIAS E MÚLTIPLAS CADEIAS ISÓCRONAS}

Ressonâncias primárias são aquelas que podem ser obtidas a partir de expansões de primeira ordem da Hamiltoniana e do mapa que descrevem o sistema. Expandindo os pulsos de onda em série de Fourier-Bessel, verifica-se que eles apresentam um número infinito de termos perturbativos. Além disso, existem grupos de termos ressonantes com o mesmo número de rotação que podem gerar ilhas diferentes na mesma região do espaço de fases. A superposição de um número infinito de termos ressonantes agindo na mesma superfície racional altera o número de cadeias de ilhas em função dos parâmetros da onda, como pode ser visto nas Refs. [50,51]. Para cada ressonância, o número de cadeias aumenta com o período e o número de onda. Para valores suficientemente elevados desses parâmetros, todas as ressonâncias apresentam mais de uma cadeia no espaço de fases, um fenômeno que não é comumente observado em sistemas twist quase integráveis. Em geral, encontra-se na literatura ressonâncias que possuem apenas uma cadeia de ilhas [14, 54].

A Hamiltoniana do sistema também revela que os termos perturbativos são simétricos e, como consequência, o número total de ilhas é par para todas as ressonâncias [50]. Portanto, o número de cadeias no espaço de fases é sempre par quando o número de ilhas em cada cadeia é ímpar. Por outro lado, quando o número de ilhas em cada cadeia é par, o número de cadeias pode ser par ou ímpar.

Construindo o espaço de parâmetros do sistema, é possível verificar como o número de cadeias de ilhas varia com o período e o número de onda. Observa-se que as fronteiras da região correspondente a um dado número de cadeias obedecem à mesma lei de potência para todas as ressonâncias [50]. Essa lei de potência está relacionada com as amplitudes dos termos ressonantes que geram as cadeias de ilhas no espaço de fases.

A partir da Hamiltoniana e do mapa do sistema, é possível estimar analiticamente a posição dos pontos periódicos no espaço de fases e as equações das fronteiras que separam duas regiões com um número diferente de cadeias de ilhas no espaço de parâmetros [50]. Conhecer essas informações é importante para a aceleração regular de partículas que ocorre nas ilhas de ressonância [15-17], pois elas permitem que as condições iniciais das partículas sejam ajustadas de modo que elas atinjam a aceleração máxima. $\mathrm{O}$ processo de aceleração regular de partículas será discutido no Capítulo 4.

Os resultados descritos neste capítulo sobre as ressonâncias primárias do sistema e o fenômeno de múltiplas cadeias isócronas foram publicados nas Refs. [50, 51] e 
apresentados em conferências internacionais.

\subsection{TEOREMA DO PONTO FIXO DE POINCARÉ-BIRKHOFF}

Como mencionado na Seção 2.3, órbitas periódicas são caracterizadas por números de rotação racionais $[14,40,58-63]$. O número de rotação determina o número de pontos periódicos e a distância entre duas iterações sucessivas da trajetória. Em sistemas integráveis, as órbitas periódicas são degeneradas, pois existe um número infinito de órbitas com o mesmo número de rotação racional [14, 40, 58, 64]. Essas órbitas estão localizadas na mesma superfície racional, que se reduz a uma linha na seção de Poincaré. Quando uma perturbação pequena é adicionada ao sistema de tal forma que ele se torna quase integrável, a degenerescência é quebrada e apenas algumas trajetórias periódicas continuam no espaço de fases $[14,40,58,64]$.

De acordo com o Teorema do Ponto Fixo de Poincaré-Birkhoff, sistemas dinâmicos quase integráveis apresentam um número par de órbitas periódicas com o mesmo número de rotação [14, 40, 54, 58, 59, 63-65], sendo metade dessas órbitas estável e metade instável. Ilhas de ressonância são formadas ao redor dos pontos periódicos estáveis e o conjunto de ilhas que circundam os pontos de uma órbita estável é conhecido como cadeia de ilhas $[14,40,58-60,63,65]$. Quando as ilhas de duas ou mais cadeias circundam trajetórias periódicas com o mesmo de número de rotação, as cadeias são chamadas de isócronas [64, 66]. Entretanto, o Teorema do Ponto Fixo de Poincaré-Birkhoff não determina o número de cadeias isócronas para cada número de rotação racional [14, 40, 58, 65].

Em sistemas não twist, o número de rotação não é uma função monotônica das variáveis [62-64]. Logo, neste caso, as seções de Poincaré podem apresentar trajetórias periódicas com o mesmo número de rotação para valores diferentes da variável de ação. As Refs. [60-63, 66, 67] mostram alguns exemplos de sistemas não twist que apresentam um par de cadeias isócronas em regiões distintas das seções de Poincaré.

Por outro lado, o número de rotação é uma função monotônica para sistemas twist [62-64], o que torna impossível a presença de cadeias isócronas em regiões diferentes das seções de Poincaré. Contudo, é possível observar mais de uma cadeia isócrona localizada na mesma posição com respeito à variável de ação. Embora alguns sistemas apresentem mais de uma cadeia [50, 51, 68, 69], em geral, encontra-se na literatura apenas uma cadeia de ilhas 
nas seções de Poincaré de sistemas twist $[14,54]$. Nas próximas seções, são analisadas as condições que dão origem à presença de múltiplas cadeias isócronas em sistemas twist.

\subsection{POSIÇÃO DAS RESSONÂNCIAS PRIMÁRIAS COM RELAÇÃO À VARIÁVEL DE AÇÃO}

A soma periódica de funções delta presente na Hamiltoniana (7) pode ser escrita como uma série de Fourier

$$
\sum_{n=-\infty}^{+\infty} \delta(t-n T)=\frac{1}{T} \sum_{l_{1}=-\infty}^{+\infty} \cos \left(\frac{2 \pi l_{1} t}{T}\right)
$$

Substituindo a relação (12) na Hamiltoniana (7) tem-se

$$
\begin{aligned}
& H=\sqrt{1+2 I}+\frac{\varepsilon}{2 T} \cos (k \sqrt{2 I} \operatorname{sen} \theta) \sum_{l_{1}=-\infty}^{+\infty} \cos \left(\frac{2 \pi l_{1} t}{T}\right), \\
& H=\sqrt{1+2 I}+\frac{\varepsilon}{2 T} \sum_{l_{1}=-\infty}^{+\infty} \cos \left(k \sqrt{2 I} \operatorname{sen} \theta-\frac{2 \pi l_{1} t}{T}\right) .
\end{aligned}
$$

Para calcular as ressonâncias primárias do sistema, é necessário expandir a Hamiltoniana (13) em primeira ordem. Isso é feito a partir da expansão da função cosseno em série de Fourier-Bessel através da seguinte relação

$$
\cos \left(k \sqrt{2 I} \operatorname{sen} \theta-\frac{2 \pi l_{1} t}{T}\right)=\sum_{l_{2}=-\infty}^{+\infty} J_{l_{2}}(k \sqrt{2 I}) \cos \left(l_{2} \theta \pm \frac{2 \pi l_{1} t}{T}\right)
$$

onde $J_{l_{2}}(k \sqrt{2 I})$ são funções de Bessel de ordem $l_{2}$ dadas por

$$
J_{l_{2}}(x)=\sum_{l_{3}=0}^{\infty} \frac{(-1)^{l_{3}}}{l_{3} !\left(l_{2}+l_{3}\right) !}\left(\frac{x}{2}\right)^{l_{2}+2 l_{3}} .
$$

Substituindo a relação (14) na expressão (13), a Hamiltoniana expandida é dada por

$$
H=\sqrt{1+2 I}+\frac{\varepsilon}{2 T} \sum_{l_{1}=-\infty}^{+\infty} \sum_{l_{2}=-\infty}^{+\infty} J_{l_{2}}(k \sqrt{2 I}) \cos \left(l_{2} \theta-\frac{2 \pi l_{1} t}{T}\right) .
$$

O sistema descrito pela Hamiltoniana (15) encontra-se em ressonância quando 
o argumento da função cosseno não varia no tempo. Isso indica que a perturbação causada pela onda não oscila no tempo e seu efeito médio sobre o sistema é não nulo. Dessa forma, a condição aproximada de ressonância primária do sistema é dada por

$$
\begin{aligned}
& \frac{\mathrm{d}}{\mathrm{d} t}\left(l_{2} \theta-\frac{2 \pi l_{1} t}{T}\right)=0, \quad l_{2} \frac{\mathrm{d} \theta}{\mathrm{d} t}=\frac{2 \pi l_{1}}{T}, \\
& l_{2} \omega_{0} \cong l_{1} \omega, \quad m r \omega_{0} \cong m s \omega, \quad r \omega_{0} \cong s \omega
\end{aligned}
$$

onde $l_{1} / l_{2}=m s / m r=s / r$, com $m, r$ e $s$ inteiros não nulos, sendo $r$ e $s$ dois números positivos e primos entre si. Além disso, $\omega=2 \pi / T$ é a frequência da onda eletrostática e foi feita a aproximação $\mathrm{d} \theta / \mathrm{d} t \cong \omega_{0}$, com $\omega_{0}$, a frequência natural do sistema, dada por

$$
\omega_{0}=\left.\frac{\mathrm{d} \theta}{\mathrm{d} t}\right|_{H=H_{0}}=\frac{\mathrm{d} H_{0}}{\mathrm{~d} I}=\frac{1}{\sqrt{1+2 I}} .
$$

De acordo com a condição (16), a perturbação devido à onda eletrostática atua de forma ressonante sobre o sistema sempre que a razão entre as frequências do movimento não perturbado e da onda for um número racional, ou seja, $\Omega=\omega_{0} / \omega=s / r$, onde $\Omega$ é o número de rotação que caracteriza a ressonância $(r, s)^{7}[14,40,58-63]$. Como $\omega$ e $\omega_{0}$ são ambas positivas, a expressão (16) é satisfeita apenas quando $l_{1}$ e $l_{2}$ possuem o mesmo sinal. Essa conclusão também pode ser obtida a partir da Hamiltoniana (15). Uma análise dos termos perturbativos dessa Hamiltoniana mostra que apenas os termos para os quais $l_{1}$ e $l_{2}$ possuem o mesmo sinal (com $l_{1} \neq 0$ e $l_{2} \neq 0$ ) estão associados com ressonâncias no espaço de fases.

Substituindo (17) em (16) é possível estimar os valores da ação $I$ ao redor dos quais estão localizadas as regiões de ressonância

$$
I_{r, s} \cong \frac{1}{2}\left(\frac{r}{s \omega}\right)^{2}-\frac{1}{2}=\frac{1}{8}\left(\frac{r T}{s \pi}\right)^{2}-\frac{1}{2}=\frac{1}{8}\left(\frac{T}{\Omega \pi}\right)^{2}-\frac{1}{2} .
$$

Logo, a posição das ressonâncias primárias no espaço de fases depende do período da onda eletrostática e do número de rotação da ressonância. A partir da expressão (18), verifica-se que o número de rotação $\Omega$ é uma função monotônica. Isso significa que o sistema em estudo

\footnotetext{
${ }^{7}$ Números de rotação racionais estão associados às ressonâncias do sistema e correspondem a órbitas periódicas. Entretanto, também existem números de rotação irracionais que correspondem a órbitas quase periódicas no espaço de fases $[13,37]$.
} 
é twist [62-64] e não pode, portanto, apresentar cadeias de ilhas isócronas em posições diferentes com relação à variável de ação $I$.

Para a ressonância $(r, s)$, verifica-se que $r$ corresponde ao número de ilhas presentes em cada cadeia localizada na posição $I_{r, s}[50,51]$. Enquanto $s$ é proporcional à variação sofrida pela partícula na variável $\theta$ entre dois pulsos de onda consecutivos, isto é, $\Delta \theta=\theta_{n+1}-\theta_{n}=2 \pi s / r=2 \pi \Omega(\bmod 2 \pi)$ no intervalo de tempo entre dois pulsos de onda consecutivos [40]. Isso significa que a cada $r$ pulsos da onda eletrostática, a partícula descreve $s$ voltas completas no plano $x \mathrm{O} y$. Portanto, quanto maior o valor de $s$, maior será a velocidade angular da partícula no plano $x \mathrm{O} y$.

É importante ressaltar que quando a amplitude $\varepsilon$ da perturbação aplicada ao sistema é elevada o suficiente, observa-se no espaço de fases outras ressonâncias além das ressonâncias primárias calculadas a partir da fórmula (18), como pode ser visto nas Figuras 3(d) e 3(f). Essas ressonâncias são de ordens mais elevadas e são geradas pelo acoplamento de ressonâncias que ocorre devido ao aumento da amplitude de perturbação [14, 40]. As ressonâncias de ordens mais elevadas não são obtidas a partir da expansão de primeira ordem realizada através da relação (14) e a análise de tais ressonâncias não faz parte dos objetivos desta tese.

\subsection{POSIÇÃO DAS RESSONÂNCIAS PRIMÁRIAS COM RELAÇÃO À VARIÁVEL ANGULAR}

Ressonâncias primárias surgem devido à interação da partícula com a onda eletrostática. Mesmo quando a amplitude $\varepsilon$ da onda é muito baixa, é possível observar a presença de ilhas de ressonância no espaço de fases. Para valores de $\varepsilon$ próximos de zero, a primeira equação do mapa (8) pode ser aproximada por

$$
I_{n+1}=I_{n}+\frac{\varepsilon k}{2} \sqrt{2 I_{n}} \cos \theta_{n} \operatorname{sen}\left(k \sqrt{2 I_{n}} \operatorname{sen} \theta_{n}\right)
$$

uma vez que o termo proporcional a $\varepsilon^{2}$ é muito menor do que o termo de primeira ordem em $\varepsilon$. Seguindo essa aproximação de primeira ordem em $\varepsilon$ nas sucessivas iterações do mapa, todos os termos de ordem $\mathrm{O}\left(\varepsilon^{2}\right)$ devem ser desprezados. Portanto, tem-se que 


$$
\begin{aligned}
& I_{n+2}=I_{n+1}+\frac{\varepsilon k}{2} \sqrt{2 I_{n+1}} \cos \theta_{n+1} \operatorname{sen}\left(k \sqrt{2 I_{n+1}} \operatorname{sen} \theta_{n+1}\right) \\
& I_{n+2}=I_{n}+\frac{\varepsilon k}{2} \sqrt{2 I_{n}} \cos \theta_{n} \operatorname{sen}\left(k \sqrt{2 I_{n}} \operatorname{sen} \theta_{n}\right)+\frac{\varepsilon k}{2} \sqrt{2 I_{n}} \cos \theta_{n+1} \operatorname{sen}\left(k \sqrt{2 I_{n}} \operatorname{sen} \theta_{n+1}\right) \\
& I_{n+r}=I_{n}+\varepsilon \sum_{j=0}^{r-1} \frac{k}{2} \sqrt{2 I_{n}} \cos \theta_{n+j} \operatorname{sen}\left(k \sqrt{2 I_{n}} \operatorname{sen} \theta_{n+j}\right)
\end{aligned}
$$

de tal forma que a somatória na expressão (20) só contenha termos de ordem zero em $\varepsilon$.

Nos pontos de período $r$ da ressonância $(r, s)^{8}$ tem-se que $I_{n+r}=I_{n} \cong I_{r, s}$, onde $I_{r, s}$ é dado pela expressão (18). Como esse resultado deve ser válido para $k \neq 0, I_{r, s} \neq 0$ e $\varepsilon$ pequeno e finito, a partir da expressão (20) verifica-se que os pontos periódicos das ressonâncias $(r, s)$ são caracterizados pela condição

$$
G_{r, s} \equiv \sum_{j=0}^{r-1} \cos \theta_{n+j} \operatorname{sen}\left(k \sqrt{2 I_{r, s}} \operatorname{sen} \theta_{n+j}\right)=0
$$

Para os pontos periódicos não perturbados, $\theta_{n+1}=\theta_{n}+2 \pi \Omega=\theta_{n}+2 \pi s / r$, de tal forma que $\theta_{n+r}=\theta_{n}+2 \pi s=\theta_{n}(\bmod 2 \pi)$. Substituindo essas expressões em (21) tem-se (novamente, em primeira ordem em $\varepsilon$ )

$$
G_{r, s}(\theta)=\sum_{j=0}^{r-1}\left\{\cos \left(\theta+\frac{2 \pi s}{r} j\right) \operatorname{sen}\left[k \sqrt{2 I_{r, s}} \operatorname{sen}\left(\theta+\frac{2 \pi s}{r} j\right)\right]\right\}=0
$$

Logo, a condição (22) fornece uma estimativa para os valores de $\theta$ nos pontos periódicos do mapa (8). Essa mesma condição também é utilizada na determinação do número de cadeias de ilhas para cada ressonância $(r, s)$. A Figura 7 foi construída para $T=2 \pi(1+$ 1/15) e $k=2$, e ela mostra os gráficos de $G_{r, s}(\theta) \times \theta$ para as ressonâncias $(4,3)$ e $(2,1)$. Os pontos de intersecção do gráfico com o eixo $\theta$ correspondem às soluções da equação (22).

A Figura 7(a) mostra a oscilação de $G_{4,3}(\theta)$ em função de $\theta$. Nessa figura, observa-se que a ressonância $(4,3)$ possui 8 pontos periódicos localizados em $\theta=0 ; \pi / 4$; $\pi / 2 ; 3 \pi / 4 ; \pi ; 5 \pi / 4 ; 3 \pi / 2 ; 7 \pi / 4$. Como cada cadeia de ilhas dessa ressonância deve possuir $r=4$ pontos elípticos e $r=4$ pontos hiperbólicos, conclui-se que a ressonância $(4,3)$ possui uma única cadeia com quatro ilhas cada. A Figura 7(b) por sua vez, corresponde à

\footnotetext{
${ }^{8}$ Pontos periódicos de período $r$ são pontos que se repetem a cada $r$ iterações do mapa. Isso significa que eles são pontos fixos do mapa $I_{n+r}=I_{n+r}\left(I_{n}, \theta_{n}\right) ; \theta_{n+r}=\theta_{n+r}\left(I_{n}, \theta_{n}\right)$.
} 
ressonância $(2,1)$ e também observa-se a presença de 8 pontos periódicos localizados em $\theta=0 ; 5 \pi / 16 ; \pi / 2 ; 11 \pi / 16 ; \pi ; 21 \pi / 16 ; 3 \pi / 2 ; 27 \pi / 16$. Entretanto, cada cadeia de ilhas da ressonância $(2,1)$ possui apenas $r=2$ pontos elípticos e $r=2$ pontos hiperbólicos. A partir desses resultados conclui-se que a ressonância $(2,1)$ possui não uma, mas duas cadeias com duas ilhas cada.

(a) Ressonância $(4,3)$

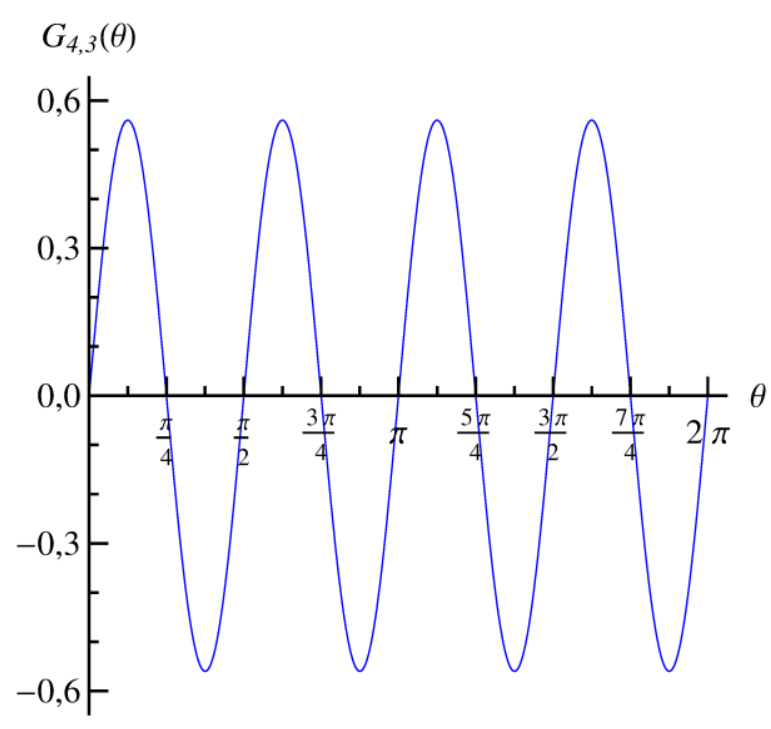

(b) Ressonância $(2,1)$

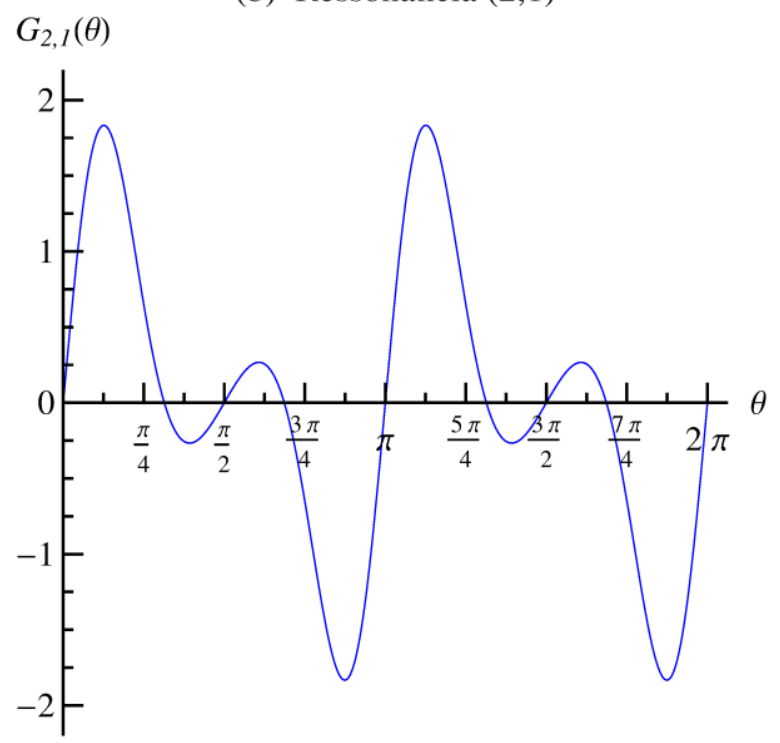

Figura 7: Gráficos de $G_{r, s}(\theta) \times \theta$ para as ressonâncias a) $(4,3)$ e b) $(2,1)$. Parâmetros utilizados: $k=2$ e $T=2 \pi(1+1 / 15)$.

Assim como a posição $I_{r, s}$ dada pela fórmula (18) varia de acordo com o período $T$ da onda eletrostática, a posição em $\theta$ obtida a partir da expressão (22) também depende do período $T$ e do número de onda $k$. Variando os parâmetros da onda eletrostática, a posição $\left(I_{r, s}, \theta\right)$ dos pontos periódicos também muda, assim como o número de pontos periódicos presentes na resonância $(r, s)$. Além disso, as fórmulas (18) e (22) são estimativas que não consideram a amplitude $\varepsilon$ da onda eletrostática. Quando $\varepsilon \rightarrow 0$, os resultados obtidos com essas expressões coincidem com os resultados obtidos numericamente a partir do mapa exato (8). Aumentando a amplitude da onda, os pontos periódicos são deslocados e os resultados obtidos a partir das fórmulas (18) e (22) perdem precisão, como pode ser visto comparando-se, por exemplo, a posição dos pontos periódicos das ressonâncias $(3,2)$ e $(4,3)$ nas Figuras 3(b) e 3(c). Entretanto, as fórmulas (18) e (22) ainda podem ser utilizadas como uma estimativa inicial para a obtenção de resultados mais precisos a partir do mapa (8).

Os únicos pontos periódicos que não são deslocados pelo aumento da 
amplitude $\varepsilon$ da onda eletrostática são aqueles localizados em $\theta=0 ; \pi$. A partir do mapa (8) verifica-se analiticamente que para $\theta=0 ; \pi, I_{n+1}=I_{n}$ e $\theta_{n+1}=\theta_{n}+T / \sqrt{1+2 I_{n}}$. Logo, na posição $I_{r, s}$ da ressonância $(r, s)$ tem-se que $\theta_{n+1}=\theta_{n}+2 \pi s / r$ para qualquer valor de $\varepsilon$.

\subsection{NÚMERO DE CADEIAS ISÓCRONAS PRESENTES NAS REGIÕES DE RESSONÂNCIAS PRIMÁRIAS}

Como mencionado na Seção 3.1, o Teorema do Ponto Fixo de PoincaréBirkhoff estabelece que qualquer ressonância primária $(r, s)$ apresenta $M r$ (sendo $M$ um número inteiro e positivo) ilhas de ressonância ao redor dos pontos periódicos estáveis [14, 40, 58-60, 63, 65]. O número $M$ indica o número de cadeias e $r$ representa o número de ilhas em cada cadeia [50,51]. O objetivo desta seção é determinar o valor de $M$, que permanece em aberto no Teorema [14, 40, 58, 65].

A maior parte dos sistemas twist quase integráveis apresentados na literatura possui uma única cadeia de ilhas em suas seções de Poincaré [14, 54]. No sistema em estudo, diversas ressonâncias primárias apresentam mais de uma cadeia no espaço de fases [50, 51], como pode ser visto na Figura 8. Essa figura mostra o espaço de fases do sistema obtido a partir do mapa (8) para $T=2,5 \pi, k=5$ e $\varepsilon=0,006$. Nessa figura, são apresentadas as ressonâncias $(1,1),(6,5),(4,3)$ e $(3,2)$, localizadas respectivamente em $I_{1,1} \cong 0,28, I_{6,5} \cong 0,62$, $I_{4,3} \cong 0,89$ e $I_{3,2} \cong 1,26$, de acordo com a estimativa (18).

Na posição $I_{6,5} \cong 0,62$, observa-se 6 ilhas da ressonância $(6,5)$. Todas essas ilhas formam uma única cadeia, pois é necessária apenas uma condição inicial para formar as 6 ilhas. Por outro lado, a ressonância $(4,3)$ localizada em $I_{4,3} \cong 0,89$ possui 8 ilhas, mas são necessárias duas condições iniciais para gerar todas as ilhas. Isso indica que a ressonância $(4,3)$ apresenta 2 cadeias com 4 ilhas cada. As ilhas de ambas as cadeias estão intercaladas no espaço de fases. As cadeias estão representadas em vermelho e verde na Figura 8 e elas também podem ser distinguidas pela forma e pelo tamanho das ilhas. As ilhas da cadeia vermelha estão centradas em $\theta=0, \pi / 2, \pi, 3 \pi / 2$, enquanto as ilhas da cadeia verde estão centradas em $\theta \cong \pi / 4,3 \pi / 4,5 \pi / 4,7 \pi / 4$. 


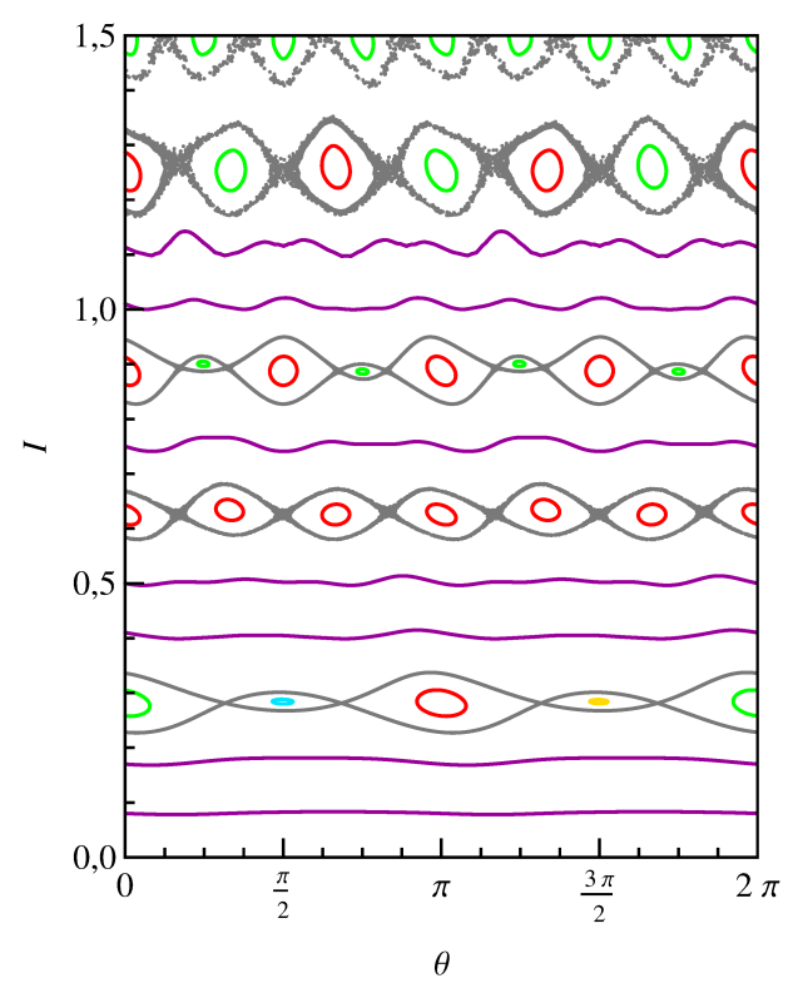

Figura 8: Espaço de fases do sistema para $T=2,5 \pi, k=5$ e $\varepsilon=0,006$. Nesta figura, são apresentadas as ressonâncias $(1,1),(6,5),(4,3)$ e $(3,2)$, localizadas respectivamente em $I_{1,1} \cong 0,28, I_{6,5} \cong 0,62, I_{4,3} \cong 0,89$ e $I_{3,2} \cong 1,26$.

Fonte: Referência [50].

A ressonância $(3,2)$ localizada ao redor de $I_{3,2} \cong 1,26$ apresenta 2 cadeias com 3 ilhas cada. As ilhas da cadeia vermelha estão centradas em $\theta=0,2 \pi / 3,4 \pi / 3$. Já as ilhas da cadeia verde estão centradas em $\theta \cong \pi / 3, \pi, 5 \pi / 3$. Finalmente, a ressonância $(1,1)$, para a qual $I_{1,1} \cong 0,28$, apresenta 4 cadeias com apenas 1 ilha cada, isto é, cada ilha da ressonância $(1,1)$ presente na Figura 8 forma uma cadeia diferente.

No sistema em estudo, condições de ressonância apropriadas são úteis no processo de aceleração regular de partículas [16, 17]. Para atingir a aceleração máxima, é necessário ajustar os parâmetros da onda eletrostática de tal forma que a partícula receba uma grande quantidade de energia da onda [16]. Além disso, as condições iniciais da partícula devem ser escolhidas de acordo com a ressonância que apresenta as melhores condições para aceleração. Por esse motivo, é importante conhecer a posição e o número de cadeias das ressonâncias $(r, s)$ e como esse número varia de acordo com os parâmetros do sistema. A Figura 9 mostra como exemplo o número de cadeias e a posição das ilhas da ressonância $(1,1)$ no espaço de fases para dois conjuntos de parâmetros da onda eletrostática.

Cada ressonância primária $(r, s)$ está localizada em uma superfície racional 
(ou linha na seção de Poincaré) $\Omega=s / r$ [14, 40, 58-63]. Entretanto, a Hamiltoniana (15) apresenta um número infinito de termos perturbativos com o mesmo número de rotação. Por exemplo, as perturbações $(2,1),(-2,-1),(4,2),(-4,-2)$, etc. são todas caracterizadas pelo número de rotação $\Omega=1 / 2$. Dessa forma, observa-se que todas as perturbações $(m r, m s)$ estão localizadas na mesma superfície racional $\Omega=s / r$. Portanto, cada uma dessas perturbações pode gerar ilhas isócronas na mesma região do espaço de fases.

Para uma ressonância $(m r, m s)$ isolada, $|m|$ representa o número de cadeias e $r$ é o número de ilhas em cada cadeia. Logo, um sistema twist que possui apenas um termo ressonante $(m r, m s)$ apresenta $|m|$ cadeias com $r$ ilhas cada na superfície racional $\Omega=s / r$. Nesse caso, o número de cadeias é constante, não dependendo dos parâmetros do sistema.

(a) $k=2$

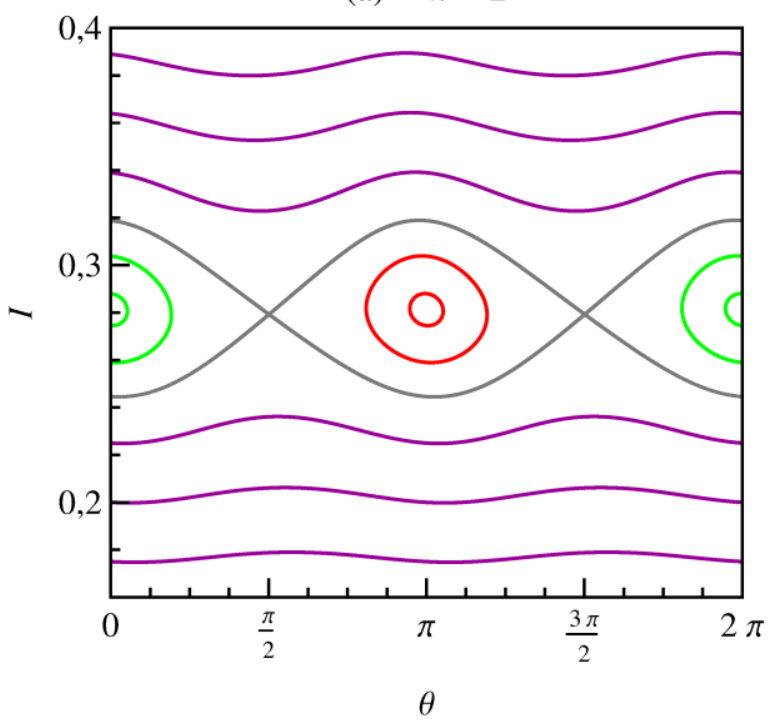

(b) $k=5$

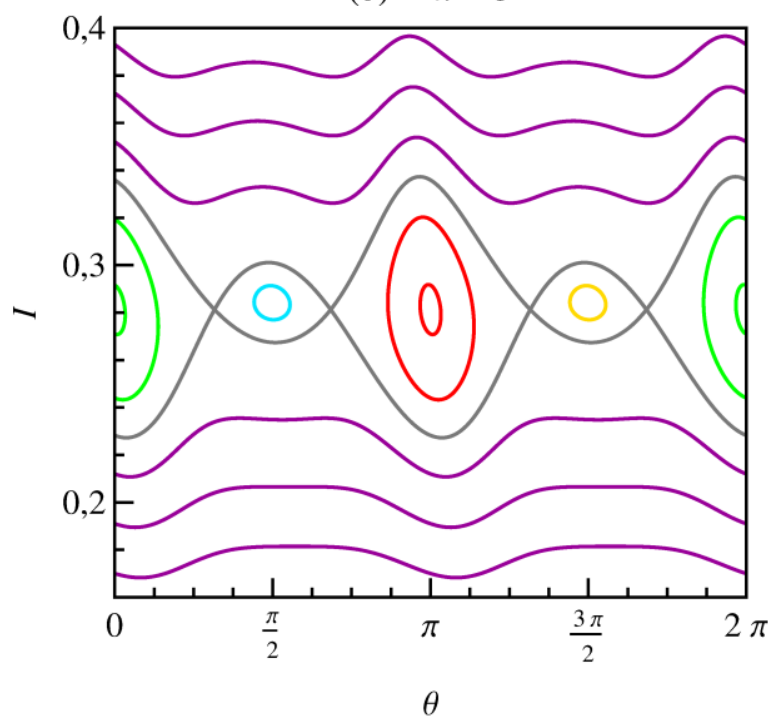

Figura 9: Espaço de fases na região da ressonância $(1,1)$ para $T=2,5 \pi, \varepsilon=0,006$ e a) $k=2$; b) $k=5$.

Para o sistema em estudo, existem infinitas perturbações $(m r, m s)$ com o mesmo número de rotação e essa superposição altera o número de cadeias no espaço de fases de acordo com os parâmetros da onda. Analisando as expressões de (16) a (22), verifica-se que elas não são funções dos números individuais $r$ e $s$. Essas expressões são na verdade funções da razão $\Omega=s / r$. Portanto, ao escolher o valor de $\Omega$ nessas expressões, estão sendo considerados todos os termos ressonantes $(m r, m s)$ localizados na superfície racional $\Omega=s / r$ e é impossível distinguir cada termo individual.

Entretanto, apenas algumas das perturbações $(m r, m s)$ geram ilhas no espaço 
de fases. O número $M$ de cadeias varia de acordo com parâmetros da onda e, em muitos casos, as $M$ cadeias são formadas pela superposição das perturbações $(m r, m s)$ para as quais $|m| \leq M[50,51]$. Embora existam exceções, a amplitude dos termos ressonantes para os quais $|m|>M$ é em geral muito menor e esses termos não contribuem para o surgimento de ilhas no espaço de fases, como será discutido adiante.

A Figura 10 mostra o número de cadeias em função do período $T$ da onda eletrostática e do número de onda $k$ para as ressonâncias $(1,1),(1,2),(2,1)$ e $(4,3)$. A figura foi gerada a partir das estimativas (18) e (22). Cada cor representa um número diferente de cadeias de ilhas, como indicado na figura. Em todos os painéis, o número de cadeias cresce com $T$ e $k$.

Para gerar a Figura 10(c), foi substituído $\Omega=s / r=1 / 2$ nas expressões (18) e (22), onde $\Omega=1 / 2$ representa todos os termos ressonantes $(2 m, m)$ da Hamiltoniana (15). Considerando uma região do espaço de fases próxima à ressonância $(2,1)$, é possível reduzir a Hamiltoniana (15) a uma Hamiltoniana local que apresenta apenas a ressonância $(2,1)$ e, portanto, é válida apenas nessa região

$$
\begin{aligned}
& H_{2,1}(I, \theta, t)=H_{0}(I)+\varepsilon V_{\text {nres }}(I, \theta, t)+\varepsilon V_{(2,1)}(I, \theta, t), \\
& H_{2,1}(I, \theta, t)=H_{0}(I)+\varepsilon V_{\text {nres }}(I, \theta, t)+\frac{\varepsilon}{2 T} \sum_{\substack{m=-\infty \\
m \neq 0}}^{+\infty} J_{2 m}(k \sqrt{2 I}) \cos \left(2 m \theta-\frac{2 \pi m t}{T}\right),
\end{aligned}
$$

onde $\varepsilon V_{\text {nres }}$ representa os termos perturbativos não ressonantes, $\varepsilon V_{(2,1)}$ são os termos perturbativos associados à ressonância $(2,1)$, e o valor de $I$ deve ser próximo de $I_{2,1}$.

A partir da Hamiltoniana (23), observa-se que a amplitude dos termos perturbativos que geram a ressonância $(2,1)$ depende de $m$. Para $m= \pm 1$, a amplitude dos termos ressonantes em (23) é proporcional à função de Bessel $J_{2}\left(k \sqrt{2 I_{2,1}}\right)=J_{2}\left(k \sqrt{(T / \pi)^{2}-1}\right)$. Para $m= \pm 2$, a amplitude dos termos ressonantes é proporcional a $J_{4}\left(k \sqrt{2 I_{2,1}}\right)=$ $J_{4}\left(k \sqrt{(T / \pi)^{2}-1}\right)$, e assim por diante. Para $T \rightarrow \pi$ ou $k \rightarrow 0$, o argumento das funções de Bessel $J_{2 m}$ tende a zero. Como para $x \rightarrow 0$ tem-se

$$
J_{2 m}(x) \approx \frac{x^{2 m}}{2^{2 m}(2 m) !},
$$

a amplitude de $J_{2}$ é muito maior do que a amplitude das funções $J_{2 m}$ para as quais $|m|>1$. 
(a) Ressonância $(1,1)$

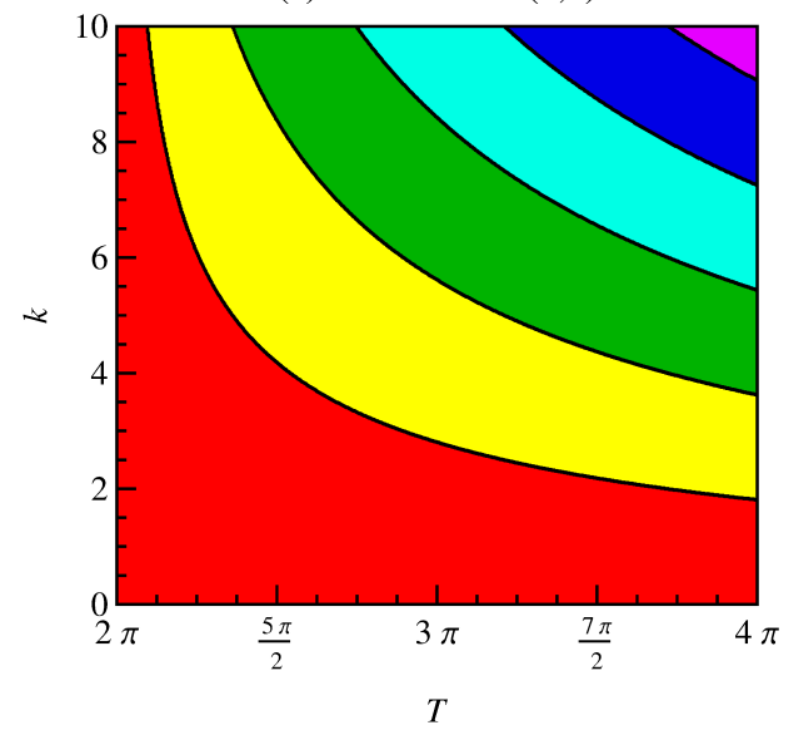

(c) Ressonância $(2,1)$

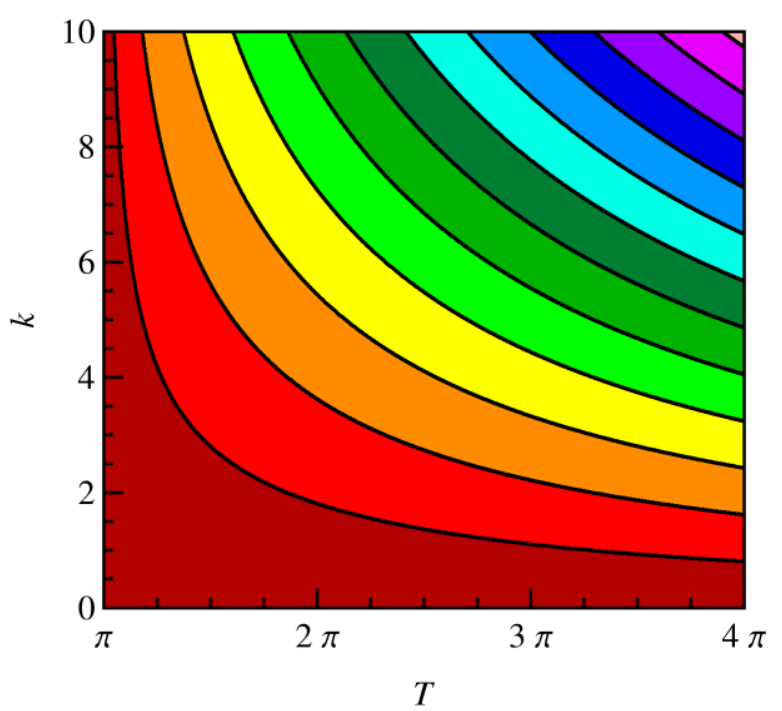

(b) Ressonância $(1,2)$

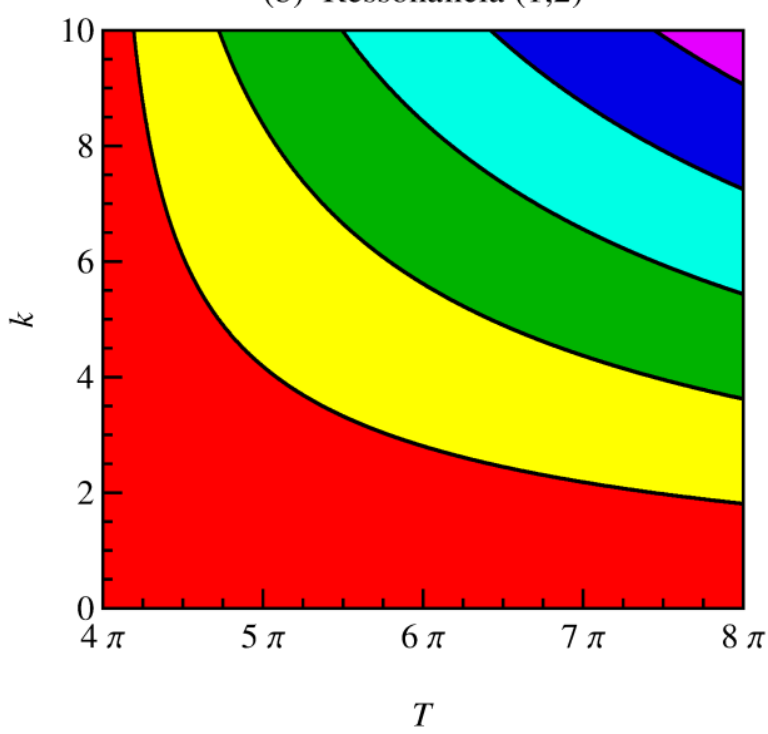

(d) Ressonância $(4,3)$

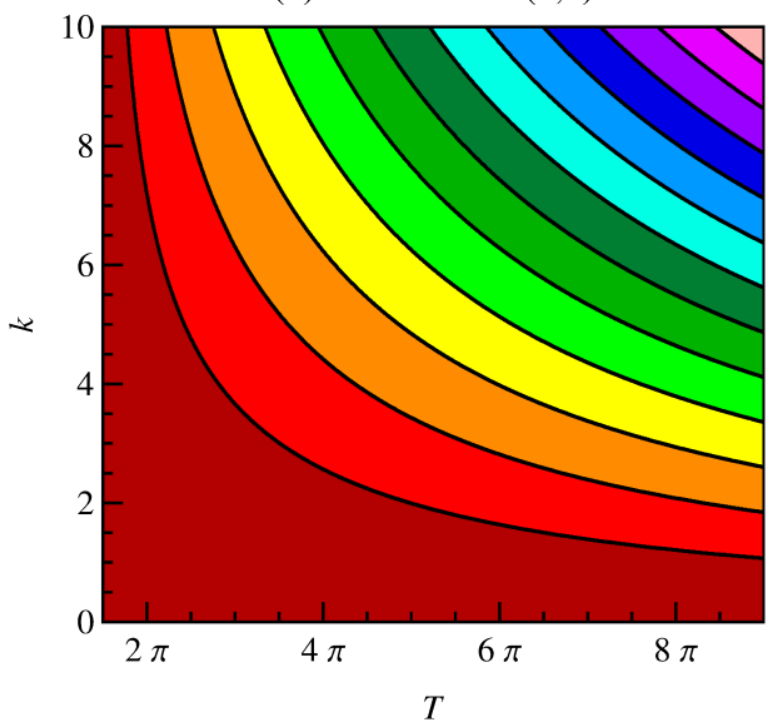

Número de cadeias isócronas

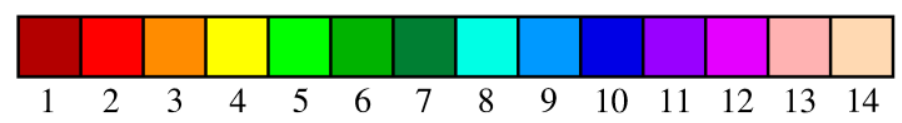

Figura 10: Número de cadeias isócronas em função de $T$ e $k$ para as ressonâncias a) $(1,1)$, b) $(1,2)$, c) $(2,1)$ e d) $(4,3)$. Cada cor representa um número diferente de cadeias, como indicado pela escala de cores. Como a variável de ação é positivo definida, a expressão (18) determina os valores de $T$ a partir dos quais cada ressonância está presente no espaço de fases.

Painéis (a) e (c) alterados de: Referência [50].

Nesse caso, apenas os termos ressonantes $m= \pm 1$ são relevantes e a ressonância $(2,1)$ apresenta uma cadeia de ilhas, como mostra a Figura 10(c).

Quando os valores de $T$ ou $k$ aumentam, a amplitude da função $J_{4}$ também aumenta e ela não pode mais ser desprezada. Nesse caso, existem quatro termos ressonantes 
( $m= \pm 1$ e $m= \pm 2$ ) com amplitudes consideráveis e o espaço de fases apresenta duas cadeias de ilhas. Conforme os valores de $T$ ou $k$ são elevados, mais termos ressonantes passam a ter amplitudes consideráveis e o número de cadeias aumenta como mostra a Figura 10(c).

A partir desses resultados verifica-se que embora a Hamiltoniana (23) possua um número infinito de perturbações $(2 m, m)$, existe um número finito $M$ de cadeias no espaço de fases para cada valor de período e de número de onda. Essas cadeias de ilhas são geradas pelas perturbações $(2 m, m)$ para as quais $|m| \leq M$, uma vez que as amplitudes dos termos ressonantes com $|m|>M$ são muito menores em comparação.

Uma análise similar pode ser feita para as demais ressonâncias exibidas na Figura 10, para as quais o número de cadeias aumenta com $T$ e $k$. Entretanto, para as ressonâncias $(r, s) \operatorname{com} r>4$, a superposição de termos ressonantes se torna mais complexa e o número de cadeias não aumenta monotonicamente com $T$ e $k$, como pode ser visto na Figura 11 para as ressonâncias $(5,2)$ e $(8,15)$. Na Figura 10, existe apenas uma região correspondente a cada número de cadeias de ilhas. A Figura 11 por sua vez apresenta no mínimo duas regiões para o mesmo número de cadeias. As regiões que correspondem a diferentes números de cadeias estão intercaladas na Figura 11 e o padrão se torna mais complicado com o aumento do número $r$ de ilhas por cadeia e dos parâmetros $T$ e $k$. Para $r=8$ como na Figura 11(b), observa-se que algumas das fronteiras que separam duas regiões com um número diferente de cadeias não são curvas lisas como nas Figuras 10 e 11(a). A Figura 11(c) mostra uma ampliação do espaço de parâmetros para a ressonância $(8,15)$, evidenciando esse comportamento.

Para $r>4$, não é mais possível afirmar que as $M$ cadeias presentes no espaço de fases são geradas apenas pelos termos ressonantes $(m r, m s)$ para os quais $|m| \leq M$. Embora $|m|$ possa ser maior do que $M$, o número de termos ressonantes $(m r, m s)$ que geram as cadeias de ilhas continua sendo finito. Por exemplo, as ilhas da ressonância $(5,2)$ correspondem os termos ressonantes $(5 m, 2 m)$. Na Figura 11(a), tem-se que as ilhas da primeira região correspondente a $M=2$ cadeias são geradas pelos termos ressonantes para os quais $m= \pm 2$. Por outro lado, as ilhas da primeira região correspondente a $M=4$ cadeias são geradas pelos termos ressonantes com $m= \pm 2$ e $m= \pm 4$. O mesmo acontece com as ilhas da segunda região correspondente a $M=2$ cadeias e com as ilhas da segunda região correspondente a $M=4$ cadeias. As ilhas dessas duas regiões também são geradas pelos termos ressonantes com $m= \pm 2$ e $m= \pm 4$. 
(a) Ressonância $(5,2)$

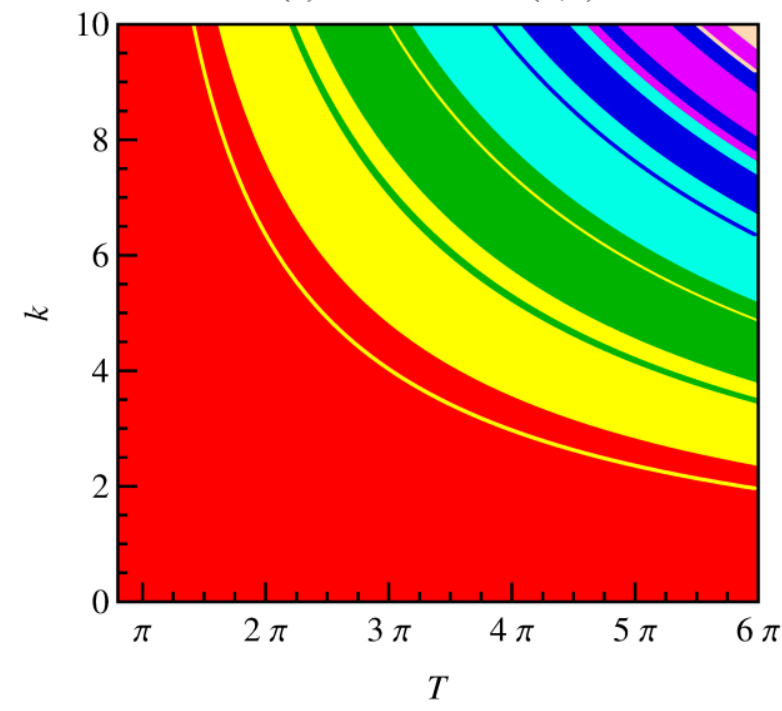

(c) Ressonância $(8,15)$ : Ampliação

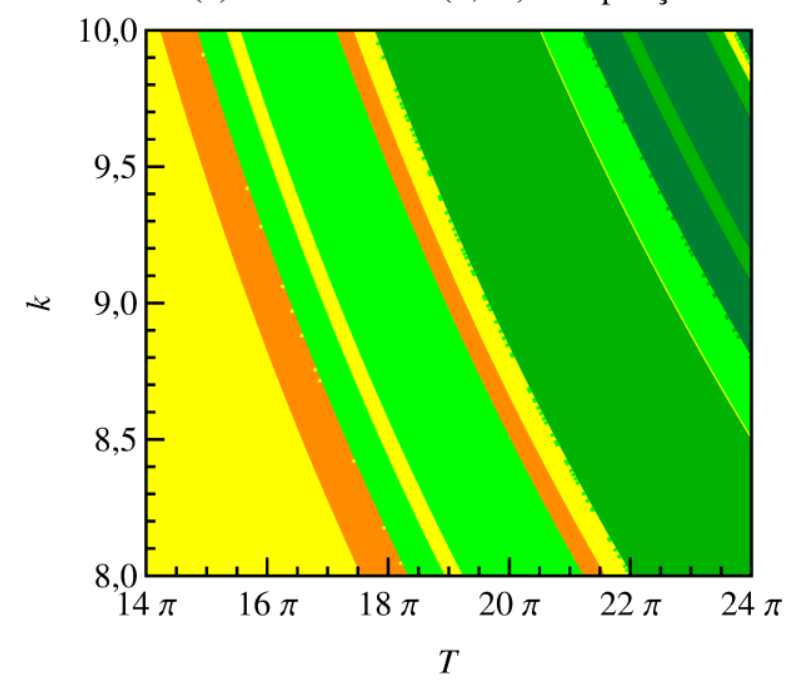

(b) Ressonância $(8,15)$

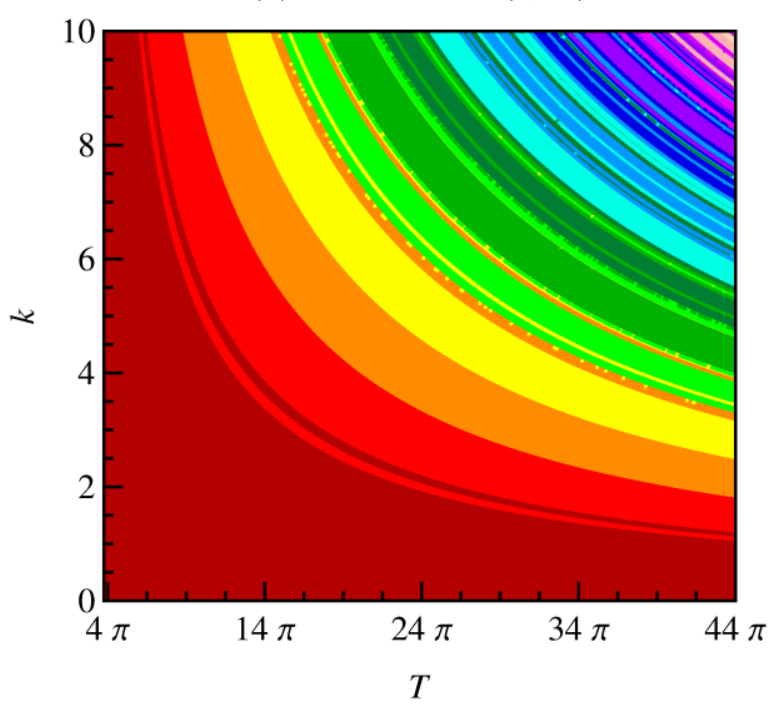

Número de cadeias isócronas
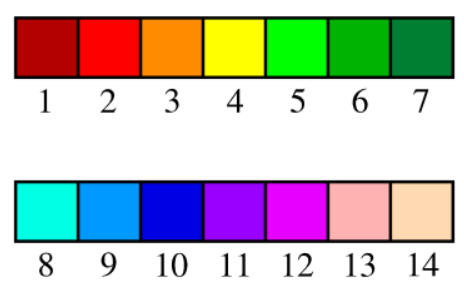

Figura 11: Número de cadeias isócronas em função de $T$ e $k$ para as ressonâncias a) $(5,2)$ e b), c) $(8,15)$. Cada cor representa um número diferente de cadeias como indicado pela escala de cores.

Painel (a) alterado de: Referência [51].

$\mathrm{O}$ argumento das funções de Bessel $J_{m r}\left(k \sqrt{2 I_{r, s}}\right)$ determina quais termos ressonantes $(m r, m s)$ atuam no sistema e qual o número $M$ de cadeias presentes no espaço de fases. Além dos parâmetros $T$ e $k, o$ argumento das funções de Bessel também é função dos números $r$ e $s$ que caracterizam a ressonância. Por esse motivo, o número de cadeias varia com os parâmetros de forma distinta para cada ressonância, como pode ser visto nas Figuras 10 e 11.

A partir das Figuras 10 e 11, observa-se que o número de cadeias isócronas para as ressonâncias $(2,1),(4,3)$ e $(8,15)$ pode ser tanto par quanto ímpar, mas o número de 
cadeias para as ressonâncias $(1,1),(1,2)$ e $(5,2)$ é sempre par. Considerando apenas os termos ressonantes da Hamiltoniana (15), verifica-se que eles são simétricos e todos os termos para os quais $l_{2}=m r$ é ímpar são cancelados aos pares [50] (veja o Apêndice A para a demonstração). Apenas os termos com $l_{2}=m r$ par atuam no sistema e eles geram um número par de ilhas para todas as ressonâncias primárias $(r, s)$. Portanto, o número de cadeias é sempre par para as ressonâncias que apresentam um número ímpar de ilhas em cada cadeia ( $r$ ímpar). Por outro lado, quando o número de ilhas em cada cadeia é par ( $r$ par), o número de cadeias pode ser par ou ímpar. Além disso, a partir da expressão (22) também é possível demonstrar que $\theta=0, \pi / 2, \pi, 3 \pi / 2$ são pontos periódicos para todas as ressonâncias primárias $(r, s)$ (veja o Apêndice B).

As Figuras 10 e 11 mostram que as ressonâncias $(r, s)$ com $r$ ímpar nunca apresentam uma única cadeia de ilhas. Mesmo para os valores mínimos dos parâmetros da onda, essas ressonâncias exibem múltiplas cadeias isócronas no espaço de fases. Como $r$ e $s$ são dois números primos entre si, o sistema em estudo possui um número muito maior de ressonâncias caracterizadas por $r$ ímpar do que por $r$ par e, como consequência, a maior parte de suas ressonâncias primárias apresenta mais de uma cadeia. Além disso, o número de cadeias aumenta com o período $T$ e com o número de onda $k$. Para valores suficientemente elevados de $T$ e $k$, espera-se que todas as ressonâncias primárias do sistema apresentem mais de uma cadeia de ilhas no espaço de fases.

A partir da condição (22), é possível calcular analiticamente o intervalo de parâmetros para um determinado número de cadeias nas ressonâncias $(1, s)$ e $(2, s)$ (veja o Apêndice C com a demonstração). Substituindo $r=1$ (ou $r=2$ ) na condição (22), obtém-se o número total de pontos periódicos em função de $k$ e $I_{1, s}\left(\right.$ ou $I_{2, s}$ ). Como as ressonâncias apresentam $M r$ ilhas ao redor dos pontos periódicos estáveis [14, 58], o intervalo de parâmetros para os quais as ressonâncias $(1, s)$ apresentam $M$ cadeias de ilhas é dado por [50]

$$
\frac{(M-2) \pi}{2 \sqrt{2 I_{1, s}}}<k<\frac{M \pi}{2 \sqrt{2 I_{1, s}}}
$$

enquanto para $r=2$, o intervalo de parâmetros é dado por [50]

$$
\frac{(M-1) \pi}{\sqrt{2 I_{2, s}}}<k<\frac{M \pi}{\sqrt{2 I_{2, s}}} .
$$


Os extremos das expressões (25) e (26) para $s=1$ correspondem às curvas pretas nas Figuras 10(a) e 10(c). Para $s=2$, os extremos da expressão (25) correspondem às curvas pretas na Figura 10(b). Cada curva preta separa duas regiões caracterizadas por um número diferente de cadeias. As estimativas analíticas (25) e (26) concordam muito bem com os resultados obtidos numericamente.

Para as ressonâncias $(r, s)$ com $r>2$, a condição (22) apresenta diversos termos na somatória e não é possível calcular analiticamente as curvas que separam duas regiões de parâmetros com um número diferente de cadeias. Entretanto, fazendo um ajuste numérico dessas curvas, verifica-se que todas elas obedecem ao mesmo tipo de lei de potência

$$
F(T) \equiv\left[\frac{1}{4}\left(\frac{r T}{s \pi}\right)^{2}-1\right]^{-1 / 2}=\frac{1}{\sqrt{2 I_{r, s}}}
$$

A expressão (27) é válida para todas as ressonâncias $(r, s)$, incluindo as ressonâncias que apresentam um comportamento mais complicado, como por exemplo a ressonância $(5,2)$ exibida na Figura 11(a). Portanto, o decaimento das curvas mencionadas anteriormente é inversamente proporcional ao argumento das funções de Bessel $J_{l_{2}}\left(k \sqrt{2 I_{r, s}}\right)$ que determinam a amplitude dos termos ressonantes na Hamiltoniana (15). Para $T>>2 s \pi / r$, a expressão (27) se reduz a uma forma mais simples dada por

$$
F(T) \approx \frac{2 s \pi}{r T}
$$

\subsection{CONCLUSÃO}

Expandindo a Hamiltoniana e o mapa do sistema em primeira ordem, foi possível analisar as ressonâncias primárias e determinar sua localização no espaço de fases, assim como o número de cadeias que elas apresentam. Para o sistema em estudo, observa-se que a maior parte das ressonâncias primárias possui mais de uma cadeia de ilhas, um fenômeno que não é comumente apresentado na literatura $[14,54]$. Além disso, o número de cadeias aumenta sem limites em função do número de onda e do período da onda que perturba o sistema. Para valores suficientemente elevados desses parâmetros, todas as regiões de 
ressonância apresentam mais de uma cadeia de ilhas no espaço de fases.

Para cada ressonância, o número de cadeias varia em função dos parâmetros, pois os pulsos de onda que perturbam o sistema apresentam um número infinito de termos ressonantes com o número de rotação que caracteriza a ressonância. Os termos ressonantes que possuem o mesmo número de rotação atuam na mesma região do espaço de fases. É a superposição de termos ressonantes que determina o número de cadeias e como esse número varia de acordo com os parâmetros.

Os resultados descritos neste capítulo não são uma característica particular do sistema em estudo. Os resultados são válidos para sistemas twist quase integráveis que apresentam um número infinito de termos ressonantes com o mesmo número de rotação. Quando o sistema apresenta um número finito de termos ressonantes com o mesmo número de rotação, o número de cadeias também varia com os parâmetros. Entretanto, o número de cadeias não aumenta sem limites e existe um conjunto finito para os possíveis números de cadeia permitidos para cada ressonância. No caso em que o sistema apresenta um único termo ressonante com o mesmo número de rotação, observa-se que o número de cadeias é completamente determinado por esse termo ressonante e ele é constante para o sistema, como pode ser observado na Ref. [51].

Para sistemas de interação onda-partícula como o analisado nesta tese, o estudo das ressonâncias primárias é importante para o processo de aceleração regular de partículas [15-17]. Para acelerar as partículas de forma regular, é preciso conhecer a posição e o número de ilhas no espaço de fases. Dessa forma, é possível ajustar as condições iniciais das partículas e fazer com que elas sigam a melhor trajetória. A aceleração regular de partículas é discutida em detalhes no próximo capítulo. 


\section{ACELERAÇÃO REGULAR DE PARTÍCULAS A PARTIR DE BAIXAS ENERGIAS INICIAIS}

Nas ilhas de ressonância presentes na Figura 3, observa-se que o valor da ação aumenta quando a onda eletrostática transfere energia para a partícula. Por esse motivo, é possível utilizar as ilhas de ressonância para fornecer energia e acelerar a partícula de forma regular. Neste capítulo, é considerada a ressonância principal do sistema. Quando o período da onda é ligeiramente maior do que período ciclotrônico, a ressonância principal está localizada no menor valor possível da ação e consequentemente no menor valor possível de energia do sistema [16]. Dessa forma, uma partícula que se move em uma das ilhas da ressonância principal pode ter uma energia inicial baixa e ser acelerada, atingindo valores mais elevados de energia. Além disso, o formato da ressonância principal difere do formato das outras ressonâncias do sistema. Sua forma triangular e o deslocamento de seus pontos hiperbólicos favorecem a aceleração da partícula [16].

Outro fator que influencia o processo de aceleração regular é o tamanho das ilhas de ressonância. O tamanho das ilhas varia de acordo com os parâmetros da onda eletrostática e, portanto, a aceleração da partícula também depende desses parâmetros. Para valores baixos dos parâmetros, a energia da partícula varia muito pouco. Elevando os parâmetros da onda, a energia máxima da partícula aumenta. Entretanto, existe um limite para o tamanho das ilhas e para o processo de aceleração regular. Quando os parâmetros da onda eletrostática se tornam elevados o suficiente, os pontos elípticos centrais das ilhas de ressonância perdem estabilidade e tornam-se hiperbólicos [16]. Quando isso acontece, as ilhas da ressonância principal são destruídas e não é mais possível acelerar a partícula de forma regular nessa região de ressonância.

Ao longo deste capítulo, são analisadas as características da ressonância principal que contribuem para a aceleração regular de partículas a partir de energias iniciais próximas à energia de repouso das partículas. Além disso, são obtidas expressões analíticas que determinam o intervalo de parâmetros da onda para os quais a aceleração regular é possível nas ilhas da ressonância principal, assim como publicado na Ref. [16]. 


\subsection{POSIÇÃO DOS PONTOS FIXOS DA RESSONÂNCIA PRINCIPAL DO SISTEMA}

A Figura 12 mostra uma ampliação do espaço de fases das Figuras 3(b) e 3(d) na região onde está localizada a ressonância (1,1). Como discutido na Seção 2.3, a ressonância $(1,1)$ é a ressonância principal do sistema e, de acordo com a expressão (18), ela está localizada no menor valor possível de ação quando o período da onda eletrostática é ligeiramente maior do que o período ciclotrônico $T_{c}=2 \pi$.

(a) $\varepsilon=2,5 \times 10^{-2}$

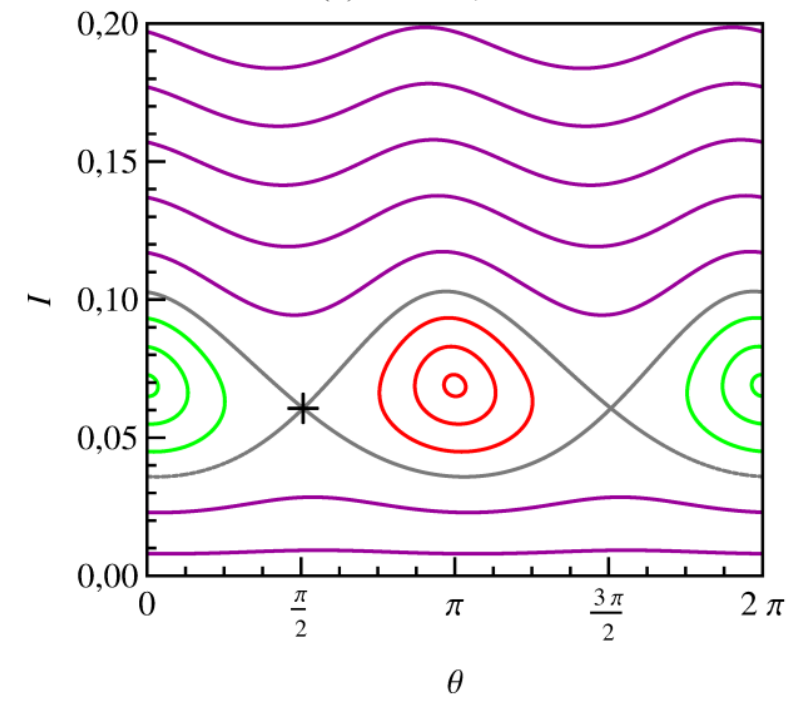

(b) $\varepsilon=0,200$

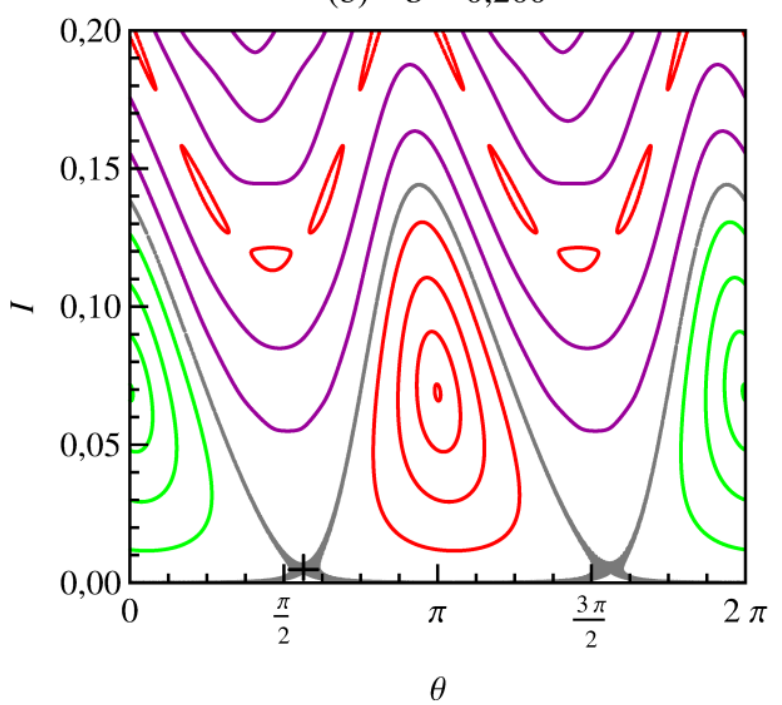

Figura 12: Ampliação do espaço de fases das Figuras 3(b) e 3(d) na região da ressonância (1,1). Parâmetros utilizados: $T=2 \pi(1+1 / 15), k=2$ e a) $\varepsilon=2,5 \times 10^{-2}$; b) $\varepsilon=0,200$.

Na Figura 12(a), a amplitude da onda eletrostática é $\varepsilon=2,5 \times 10^{-2}$ e observa-se que as ilhas da ressonância $(1,1)$ possuem a forma usual das ressonâncias do pêndulo, com os pontos elípticos e hiperbólicos localizados aproximadamente no mesmo nível de ação $I$. Devido à posição da ressonância no espaço de fases, a ação inicial $I_{0}$ de uma partícula que se move nessas ilhas deve ser superior a 0,036. Além disso, a ação, e consequentemente a energia da partícula, não variam muito com o tempo pois a largura das ilhas é pequena.

Aumentando a amplitude $\varepsilon$ como na Figura 12(b), os pontos hiperbólicos são deslocados para valores menores de ação, enquanto os pontos elípticos permanecem no mesmo local. Isso faz com que a ressonância passe a apresentar uma forma triangular, com os pontos hiperbólicos muito próximos do eixo $I=0$. Essa característica da ressonância $(1,1)$ é 
extremamente importante pois contribui para a aceleração regular de partículas a partir de baixas energias [16]. Para $\varepsilon=0,200$, a energia inicial da partícula pode ser próxima de sua energia de repouso e ainda assim a partícula será acelerada, atingindo energias mais elevadas do que no caso $\varepsilon=2,5 \times 10^{-2}$.

Além do deslocamento em $I$, o aumento da amplitude da onda eletrostática também provoca o deslocamento dos pontos hiperbólicos da ressonância $(1,1)$ com relação à variável $\theta$. Para $\varepsilon=2,5 \times 10^{-2}$ como na Figura 12(a), os pontos hiperbólicos estão localizados em $(I, \theta) \cong(0,061 ; \pi / 2)$ e $(I, \theta) \cong(0,061 ; 3 \pi / 2)$, isto é, os pontos hiperbólicos estão próximos do valor aproximado $I_{1,1} \cong 0,069$ dado pela equação (18) e $\theta=\pi / 2 ; 3 \pi / 2$ dado pela expressão (22). Entretanto, para $\varepsilon=0,200$ como na Figura 12(b), os pontos hiperbólicos estão localizados em $(I, \theta) \cong(0,004 ; 9 \pi / 16)$ e $(I, \theta) \cong(0,004 ; 25 \pi / 16)$. Para valores elevados da amplitude $\varepsilon$, observa-se que os pontos hiperbólicos se afastam muito do valor aproximado $I_{1,1} \cong 0,069$ e estão localizados próximos ao eixo $I=0$. Além disso, os pontos hiperbólicos se encontram à direita dos valores aproximados $\theta=\pi / 2 ; 3 \pi / 2$, embora $\mathrm{o}$ deslocamento com relação à variável $\theta$ não seja tão expressivo quanto o deslocamento com relação à variável de ação $I$.

A Figura 13 mostra em detalhes a posição dos pontos hiperbólicos indicados por uma cruz preta na Figura 12 para os dois valores de $\varepsilon$ analisados (observe a diferença na escala dos eixos nos dois quadros). Conhecer a posição exata dos pontos fixos da ressonância $(1,1)$ é importante para que as condições iniciais da partícula sejam ajustadas de modo que sua aceleração seja máxima. Observa-se que o mapa aproximado (9) e a expansão (22) fornecem a posição dos pontos hiperbólicos como $\theta=\pi / 2 ; 3 \pi / 2$ para qualquer valor de $\varepsilon$. Entretanto, a forte não linearidade do mapa exato (8) com relação à amplitude $\varepsilon$ desloca esses pontos fixos e, portanto, sua posição só pode ser determinada com precisão através do mapa exato (8).

Nota-se que embora os pontos hiperbólicos da ressonância $(1,1)$ sejam deslocados pelo aumento da amplitude da onda, os pontos elípticos permanecem na mesma posição independente do valor de $\varepsilon$. Analisando o mapa (8), é possível determinar analiticamente que $\theta_{n}=0 ; \pi$ são pontos periódicos do mapa para qualquer valor de $\varepsilon$. Para a ressonância $(1,1)$ e $\theta_{n}=0 ; \pi$, tem-se que $I_{n+1}=I_{n} \approx I_{1,1}$, onde $I_{1,1}$ é dado pela equação (18), e $\theta_{n+1} \approx \theta_{n}+T / \sqrt{1+2 I_{1,1}}$. Portanto, $\theta_{n+1} \approx \theta_{n}+2 \pi T / T=\theta_{n}$.

Além disso, observa-se que a topologia da Figura 12 é similar em contextos 
(a) $\varepsilon=2,5 \times 10^{-2}$

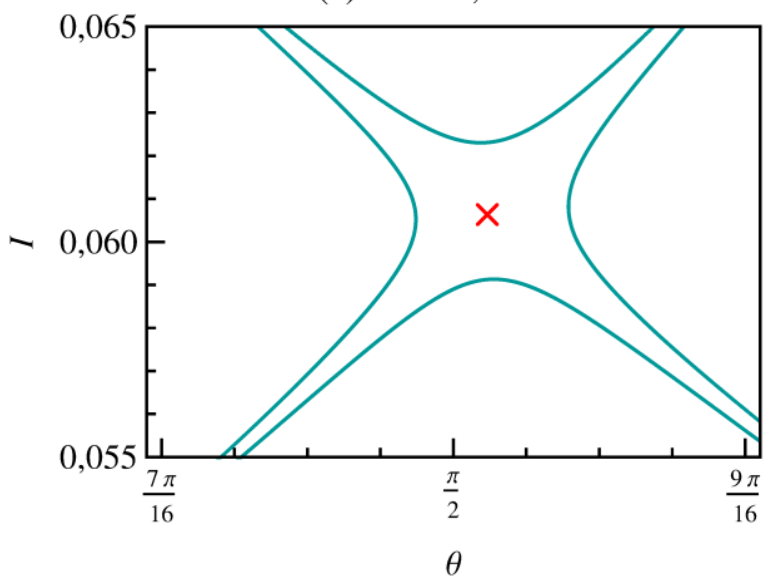

(b) $\varepsilon=0,200$

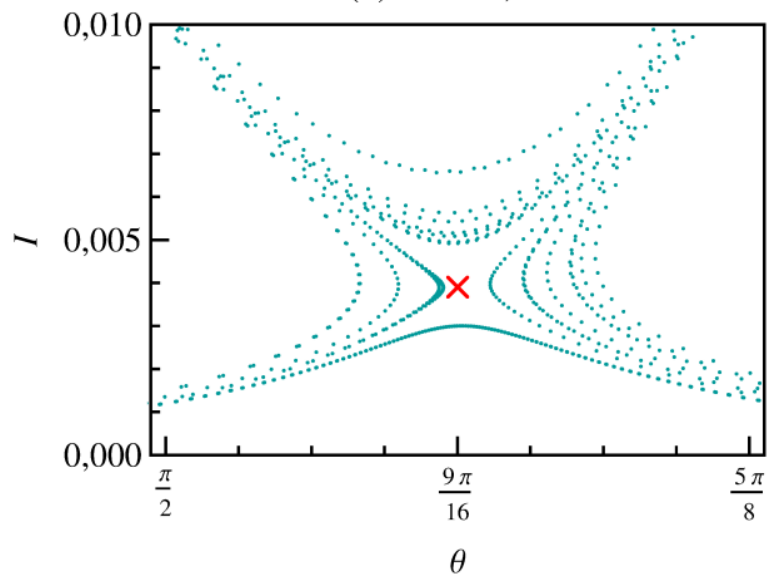

Figura 13: Posição dos pontos hiperbólicos da ressonância $(1,1)$ indicados por uma cruz preta na Figura 12. O ponto hiperbólico está representado por um $\times$ vermelho nos dois quadros. Parâmetros utilizados: $T=2 \pi(1+$ 1/15), $k=2$ e a) $\varepsilon=2,5 \times 10^{-2}$; b) $\varepsilon=0,200$.

relacionados e que envolvem perturbações dependentes do tempo e uniformes no espaço [70], embora o mapa exato (8) mostre que os pontos hiperbólicos não coincidem nos dois casos.

\subsection{PONTOS HIPERBÓLICOS DA RESSONÂNCIA PRINCIPAL LOCALIZADOS SOBRE O EIXO $I=0$}

Numericamente é possível calcular a largura máxima das ilhas da ressonância $(1,1)$ presentes na Figura 12. A partir dessa informação determina-se a energia, o momento e a velocidade adimensionais mínimos e máximos da partícula nas ilhas de ressonância, como mostra a Tabela 1. A energia da partícula é calculada a partir da Hamiltoniana (7), $E \approx \sqrt{1+2 I}$, e o momento a partir da transformação canônica, $p_{x}=\sqrt{2 I} \cos \theta$. Utilizando a expressão (2) e a definição de momento adimensional $p_{x} / m c \rightarrow p_{x}$ apresentada na Seção 2.1, é possível calcular a velocidade adimensional $v_{x} / c \rightarrow v_{x}$ da partícula como $v_{x}=p_{x}\left(1+p_{x}^{2}\right)^{-1 / 2}$ [16].

Quando a partícula está em repouso, sua energia adimensional é igual a um. A partir dos dados da Tabela 1, observa-se que a energia mínima da partícula para $\varepsilon=0,200$ é próxima de sua energia de repouso. Além disso, a energia máxima da partícula é maior para $\varepsilon=0,200$ do que para $\varepsilon=2,5 \times 10^{-2}$. Isso significa que o processo de aceleração regular 
melhora quando os pontos hiperbólicos da ressonância $(1,1)$ estão mais próximos do eixo $I=0$. Dessa forma, a aceleração máxima da partícula será obtida quando os pontos hiperbólicos estiverem localizados exatamente sobre o eixo $I=0$ [16].

Para a ressonância $(1,1)$, os pontos hiperbólicos são pontos fixos do mapa (8), isto é, para os pontos hiperbólicos tem-se $I_{n+1}=I_{n}$ e $\theta_{n+1}=\theta_{n}(\bmod 2 \pi)$, que também pode ser escrito como $\theta_{n+1}=\theta_{n}+2 j \pi$, com $j$ um número inteiro. Utilizando essa condição, determinam-se os parâmetros da onda eletrostática para os quais os pontos hiperbólicos da ressonância principal tocam o eixo $I=0$.

Tabela 1 - Energia, módulo do momento e módulo da velocidade adimensionais mínimos e máximos da partícula nas ilhas da ressonância $(1,1)$ presentes na Figura 12.

\begin{tabular}{|l|c|c|}
\hline & Figura 12(a) & Figura 12(b) \\
\hline Amplitude $\varepsilon$ da Onda Eletrostática & $2,5 \times 10^{-2}$ & 0,200 \\
Energia Adimensional Mínima & 1,035 & 1,001 \\
Energia Adimensional Máxima & 1,098 & 1,134 \\
Ganho de Energia Adimensional & 0,063 & 0,133 \\
Momento Adimensional Mínimo & 0,267 & 0,043 \\
Momento Adimensional Máximo & 0,452 & 0,526 \\
Ganho de Momento Adimensional & 0,186 & 0,483 \\
Velocidade Adimensional Mínima & 0,258 & 0,043 \\
Velocidade Adimensional Máxima & 0,412 & 0,466 \\
Ganho de Velocidade Adimensional & 0,154 & 0,423 \\
\hline
\end{tabular}

Calculando o limite de $I_{n} \rightarrow 0$ na segunda equação do mapa (8) tem-se [16]

$$
\begin{aligned}
& \theta_{n+1}=\lim _{I_{n} \rightarrow 0}\left\{\operatorname{arctg}\left[\frac{2 \sqrt{2 I_{n}} \operatorname{sen} \theta_{n}}{2 \sqrt{2 I_{n}} \cos \theta_{n}+\varepsilon k \operatorname{sen}\left(k \sqrt{2 I_{n}} \operatorname{sen} \theta_{n}\right)}\right]+\frac{T}{\sqrt{1+2 I_{n}}}\right\}, \\
& \theta_{n}+2 j \pi=\lim _{I_{n} \rightarrow 0}\left\{\operatorname{arctg}\left[\frac{2 \operatorname{sen} \theta_{n}}{2 \cos \theta_{n}+\varepsilon k^{2} \operatorname{sen} \theta_{n} \cos \left(k \sqrt{2 I_{n}} \operatorname{sen} \theta_{n}\right)}\right]+\frac{T}{\sqrt{1+2 I_{n}}}\right\}, \\
& \theta_{n}+2 j \pi=\operatorname{arctg}\left[\frac{2 \operatorname{sen} \theta_{n}}{2 \cos \theta_{n}+\varepsilon k^{2} \operatorname{sen} \theta_{n}}\right]+T, \\
& \theta_{n}+2 j \pi=\operatorname{arctg}\left[\frac{\operatorname{tg} \theta_{n}}{1+0,5 \varepsilon k^{2} \operatorname{tg} \theta_{n}}\right]+T .
\end{aligned}
$$


Utilizando algumas relações trigonométricas, a expressão (29) torna-se [16]

$$
\begin{aligned}
& \frac{\operatorname{sen}\left(\theta_{n}-T\right)}{\cos \left(\theta_{n}-T\right)}=\frac{\operatorname{tg} \theta_{n}}{1+0,5 \varepsilon k^{2} \operatorname{tg} \theta_{n}}, \\
& \frac{\operatorname{sen} \theta_{n} \cos T-\operatorname{sen} T \cos \theta_{n}}{\cos \theta_{n} \cos T+\operatorname{sen} \theta_{n} \operatorname{sen} T}=\frac{\operatorname{tg} \theta_{n}}{1+0,5 \varepsilon k^{2} \operatorname{tg} \theta_{n}}, \\
& \frac{\operatorname{tg} \theta_{n}-\operatorname{tg} T}{1+\operatorname{tg} \theta_{n} \operatorname{tg} T}=\frac{\operatorname{tg} \theta_{n}}{1+0,5 \varepsilon k^{2} \operatorname{tg} \theta_{n}}, \\
& \operatorname{tg}^{2} \theta_{n}\left(\frac{\varepsilon k^{2}}{2}-\operatorname{tg} T\right)-\frac{\varepsilon k^{2}}{2} \operatorname{tg} \theta_{n} \operatorname{tg} T-\operatorname{tg} T=0, \\
& \operatorname{tg} \theta_{n}=\frac{\varepsilon k^{2} \operatorname{tg} T \pm \sqrt{\varepsilon^{2} k^{4} \operatorname{tg}^{2} T+8 \varepsilon k^{2} \operatorname{tg} T-16 \operatorname{tg}^{2} T}}{2\left(\varepsilon k^{2}-2 \operatorname{tg} T\right)}
\end{aligned}
$$

A expressão (30) só possui soluções reais para

$$
\varepsilon^{2} k^{4} \operatorname{tg}^{2} T+8 \varepsilon k^{2} \operatorname{tg} T-16 \operatorname{tg}^{2} T \geq 0
$$

ou seja,

$$
\varepsilon^{2} k^{4} \operatorname{tg} T+8 \varepsilon k^{2}-16 \operatorname{tg} T \geq 0 \quad \text { e } \quad \operatorname{tg} T \geq 0
$$

ou

$$
\varepsilon^{2} k^{4} \operatorname{tg} T+8 \varepsilon k^{2}-16 \operatorname{tg} T \leq 0 \quad \text { e } \quad \operatorname{tg} T \leq 0
$$

Logo, o menor valor de $\varepsilon k^{2}$ para o qual os pontos hiperbólicos tocam o eixo $I=0$ é dado por [16]

$$
\left(\varepsilon k^{2}\right)_{I=0}=\frac{4\left(\sqrt{1+\operatorname{tg}^{2} T}-1\right)}{\operatorname{tg} T} \quad(\operatorname{com} \operatorname{tg} T>0)
$$

ou

$$
\left(\varepsilon k^{2}\right)_{I=0}=\frac{-4\left(\sqrt{1+\operatorname{tg}^{2} T}+1\right)}{\operatorname{tg} T} \quad(\operatorname{com} \operatorname{tg} T<0)
$$

lembrando que os parâmetros da onda eletrostática $\left(\varepsilon k^{2}\right)_{I=0}$ não podem ser negativos.

Para $T=2 \pi(1+1 / 15)$ como nas figuras dos Capítulos 2 e $4,\left(\varepsilon k^{2}\right)_{I=0} \cong 0,850$. Por esse motivo, na Figura 12(b) foram escolhidos os parâmetros $k=2$ e $\varepsilon=0,200$ que 
correspondem a $\varepsilon k^{2}=0,800$. Esses parâmetros estão próximos do limite para o qual os pontos hiperbólicos da ressonância $(1,1)$ tocam o eixo $I=0$.

Para um dado valor do período $T$ e do número de onda $k$, os pontos hiperbólicos da ressonância $(1,1)$ estão localizados sobre o eixo $I=0$ para valores da amplitude $\varepsilon$ iguais ou superiores ao valor dado pelas expressões (31). Como visto na Seção 2.3 e na Figura 3, o aumento da amplitude $\varepsilon$ da onda eletrostática provoca o aumento das regiões de comportamento caótico e a consequente destruição de trajetórias regulares no espaço de fases. Por esse motivo, para $T$ e $k$ fixos, deve ser escolhido o menor valor possível de $\varepsilon$ que satisfaça a fórmula (31) para que a partícula seja acelerada de forma regular nas ilhas da ressonância $(1,1)$.

\subsection{BIFURCAÇÃO DOS PONTOS ELÍPTICOS DA RESSONÂNCIA PRINCIPAL}

Quando os pontos hiperbólicos da ressonância $(1,1)$ estão localizados sobre o eixo $I=0$, o valor máximo da ação nas ilhas de ressonância é aproximadamente o dobro do valor de $I$ nos pontos elípticos [16], como pode ser visto na Figura 12(b). A topologia dessa figura sugere que uma das formas de melhorar a aceleração regular da partícula seria aumentar o período $T$ da onda eletrostática, uma vez que a posição dos pontos elípticos localizados em $\theta=0 ; \pi$ aumenta com $T$ conforme a equação (18). Mesmo para valores elevados do período é possível utilizar a expressão (31) e ajustar os valores de $k$ e $\varepsilon$ de tal forma que os pontos hiperbólicos estejam localizados sobre o eixo $I=0$.

Entretanto, existe um limite para esse procedimento, pois quando a amplitude da onda eletrostática atinge valores elevados o suficiente, os pontos elípticos centrais da ressonância principal perdem estabilidade [16]. Os pontos elípticos (estáveis) localizados em $\theta=0 ; \pi$ tornam-se pontos hiperbólicos (instáveis) e as duas cadeias de ilhas deixam de existir. Logo, o processo de aceleração regular só é possível quando os pontos hiperbólicos da ressonância $(1,1)$ atingem o eixo $I=0$ antes dos pontos elípticos em $\theta=0 ; \pi$ perderem estabilidade.

Quando os pontos fixos localizados em $\theta=0 ; \pi$ tornam-se hiperbólicos, uma ressonância de ordem mais elevada surge no lugar da ressonância primária $(1,1)$, sendo que a nova ressonância possui duas cadeias com duas ilhas cada. Esse processo é conhecido como 
bifurcação com dobramento de período, pois uma ressonância de período $r$ deixa de existir e surge uma ressonância de período $2 r$ em seu lugar no espaço de fases [14, 40]. A bifurcação dos pontos elípticos da ressonância $(1,1)$ pode ser vista na Figura 14 construída para $T=2 \pi(1+1 / 15)$ e $k=2$. Na Figura 14(a), $\varepsilon=2,500$ e a ressonância principal ainda está presente no espaço de fases. Contudo, as ilhas dessa ressonância estão muito pequenas (veja a escala dos eixos, principalmente do eixo $\theta$ ), alongadas em uma direção e estreitas na outra direção. Isso acontece porque os parâmetros utilizados estão próximos dos valores necessários para que o ponto elíptico sofra uma bifurcação. Quanto mais próximo dos parâmetros de bifurcação, mais as ilhas da ressonância $(1,1)$ serão alongadas em uma direção e comprimidas na direção oposta.

(a) $\varepsilon=2,500$

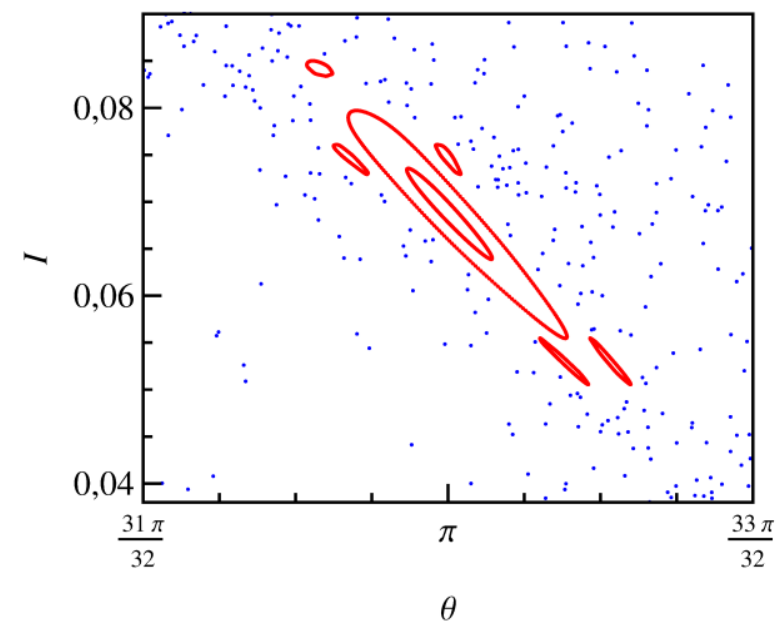

(b) $\varepsilon=2,750$

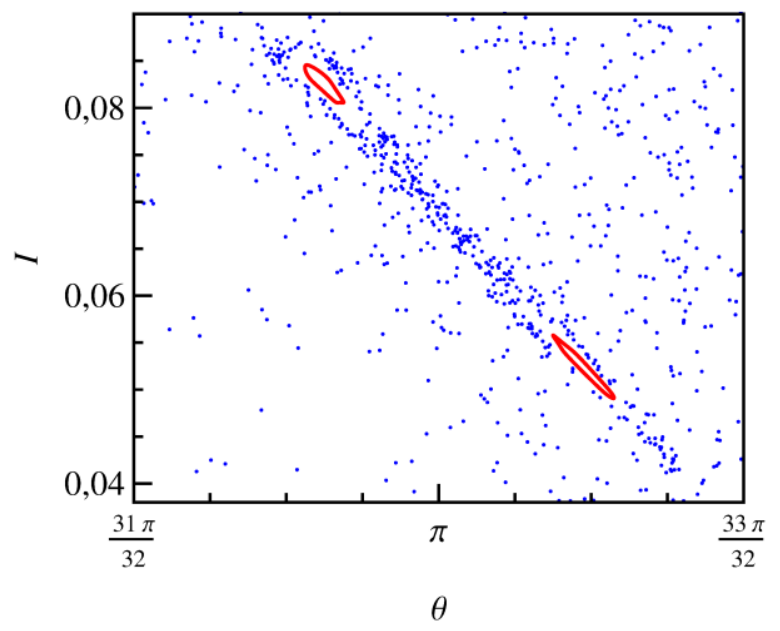

Figura 14: Bifurcação com dobramento de período dos pontos elípticos da ressonância principal do sistema. Parâmetros utilizados: $T=2 \pi(1+1 / 15), k=2$ e a) $\varepsilon=2,500$ (antes da bifurcação); b) $\varepsilon=2,750$ (depois da bifurcação).

Aumentando o valor de $\varepsilon$ para 2,750 como na Figura 14(b), a ressonância $(1,1)$ não está mais presente no espaço de fases. Os pontos fixos $(I, \theta)=\left(\mathrm{I}_{1,1} ; 0\right) \cong$ $(0.069 ; 0)$ e $(I, \theta)=\left(\mathrm{I}_{1,1} ; \pi\right) \cong(0.069 ; \pi)$ tornaram-se hiperbólicos e quatro novas ilhas de ressonância surgiram no espaço de fases, sendo uma cadeia com duas ilhas ao redor de cada ponto fixo que se bifurcou. Observa-se que essas ilhas de ressonância secundária são muito menores do que as ilhas de ressonância primária, e elas estão localizadas na região que correspondia às extremidades das ilhas da antiga ressonância (1,1). As novas ilhas de ressonância da Figura 14(b) estão imersas no mar de caos. Entretanto, as trajetórias caóticas são mais densas ao redor das ilhas, indicando que as órbitas caóticas ficam presas ao redor da 
ressonância durante um grande número de iterações do mapa antes de escaparem e atingirem valores elevados de ação $I$.

É possível calcular analiticamente os parâmetros da onda eletrostática para os quais os pontos elípticos da ressonância $(1,1)$ localizados em $\theta=0 ; \pi$ sofrem uma bifurcação com dobramento de período. Primeiramente, considera-se que $I_{n}$ e $\theta_{n}$ estão muito próximos de um dos pontos elípticos $I^{*}=\mathrm{I}_{1,1}$ e $\theta^{*}=0 ; \pi$. Nesse caso, é possível escrever $I_{n}=I^{*}+\delta I_{n}$ e $\theta_{n}=\theta^{*}+\delta \theta_{n}$, com $\delta I_{n} \rightarrow 0$ e $\delta \theta_{n} \rightarrow 0$. Além disso, muito próximo dos pontos fixos o mapa do sistema pode ser considerado linear e, portanto, tem-se

$$
\begin{aligned}
& \left(\begin{array}{l}
I_{n+1} \\
\theta_{n+1}
\end{array}\right)=J\left(\begin{array}{l}
I_{n} \\
\theta_{n}
\end{array}\right), \\
& \left(\begin{array}{l}
I^{*}+\delta I_{n+1} \\
\theta^{*}+\delta \theta_{n+1}
\end{array}\right)=J\left(\begin{array}{l}
I^{*}+\delta I_{n} \\
\theta^{*}+\delta \theta_{n}
\end{array}\right), \\
& \left(\begin{array}{l}
I^{*} \\
\theta^{*}
\end{array}\right)+\left(\begin{array}{l}
\delta I_{n+1} \\
\delta \theta_{n+1}
\end{array}\right)=J\left(\begin{array}{l}
I^{*} \\
\theta^{*}
\end{array}\right)+J\left(\begin{array}{l}
\delta I_{n} \\
\delta \theta_{n}
\end{array}\right),
\end{aligned}
$$

onde $J$ é o mapa linearizado ao redor de um dos pontos elípticos e $J$ é função apenas dos parâmetros do sistema, não dependendo das variáveis $I$ e $\theta$. Como $I^{*}$ e $\theta^{*}$ são pontos fixos do mapa, tem-se $J\left(I^{*}\right)=I^{*}$ e $J\left(\theta^{*}\right)=\theta^{*}$. Logo, ao redor dos pontos fixos o mapa do sistema é aproximadamente dado por

$$
\left(\begin{array}{l}
\delta I_{n+1} \\
\delta \theta_{n+1}
\end{array}\right)=J\left(\begin{array}{l}
\delta I_{n} \\
\delta \theta_{n}
\end{array}\right) .
$$

Analisando a estabilidade do mapa linearizado (32) ao redor dos pontos elípticos $I^{*}=\mathrm{I}_{1,1}$ e $\theta^{*}=0 ; \pi$ da ressonância $(1,1)$, determinam-se os parâmetros da onda eletrostática para os quais ocorre a bifurcação com dobramento de período. O mapa linearizado $J$ corresponde à matriz Jacobiana do mapa (8) calculada nos pontos elípticos $I^{*}=\mathrm{I}_{1,1}$ e $\theta^{*}=0 ; \pi$

$$
J=\left(\begin{array}{ll}
\frac{\partial I_{n+1}}{\partial I_{n}} & \frac{\partial I_{n+1}}{\partial \theta_{n}} \\
\frac{\partial \theta_{n+1}}{\partial I_{n}} & \frac{\partial \theta_{n+1}}{\partial \theta_{n}}
\end{array}\right)_{\left(I^{*}, \theta^{*}\right)}
$$




$$
J=\left(\begin{array}{cc}
1 & \varepsilon k^{2} I_{1,1} \\
\frac{-T}{\left(1+2 I_{1,1}\right)^{3 / 2}} & 1-\frac{\varepsilon k^{2} T I_{1,1}}{\left(1+2 I_{1,1}\right)^{3 / 2}}
\end{array}\right)
$$

De acordo com a equação (18), $I_{1,1}=T^{2} / 8 \pi^{2}-0,5$ e, portanto, a expressão (33) pode ser reescrita como

$$
J=\left(\begin{array}{cc}
1 & \frac{\varepsilon k^{2} T^{2}}{8 \pi^{2}}-\frac{\varepsilon k^{2}}{2} \\
-\frac{8 \pi^{3}}{T^{2}} & 1-\pi \varepsilon k^{2}+\frac{4 \pi^{3} \varepsilon k^{2}}{T^{2}}
\end{array}\right)
$$

A estabilidade dos pontos fixos é determinada a partir dos autovalores $\lambda$ da matriz Jacobina (34)

$$
\begin{aligned}
& \operatorname{det}\left[J-\lambda\left(\begin{array}{ll}
1 & 0 \\
0 & 1
\end{array}\right)\right]=0 \\
& \left|\begin{array}{cc}
1-\lambda & \frac{\varepsilon k^{2} T^{2}}{8 \pi^{2}}-\frac{\varepsilon k^{2}}{2} \\
-\frac{8 \pi^{3}}{T^{2}} & 1-\pi \varepsilon k^{2}+\frac{4 \pi^{3} \varepsilon k^{2}}{T^{2}}-\lambda
\end{array}\right|=0, \\
& \lambda^{2}+\left(\pi \varepsilon k^{2}-2-\frac{4 \pi^{3} \varepsilon k^{2}}{T^{2}}\right) \lambda+1=0, \\
& \lambda_{1,2}=\frac{2 \pi^{3} \varepsilon k^{2}}{T^{2}}+1-\frac{\pi \varepsilon k^{2}}{2} \pm \sqrt{\left(\frac{\pi \varepsilon k^{2}}{2}-1-\frac{2 \pi^{3} \varepsilon k^{2}}{T^{2}}\right)^{2}-1}
\end{aligned}
$$

Se $\lambda_{1,2}$ forem complexos conjugados, então $\left|\lambda_{1,2}\right|=1$ e os pontos fixos $I^{*}=\mathrm{I}_{1,1}=T^{2} / 8 \pi^{2}-$ 0,5 e $\theta^{*}=0 ; \pi$ serão elípticos, ou seja, os pontos fixos serão estáveis. Por outro lado, se $\lambda_{1,2}$ forem reais, então $\left|\lambda_{1}\right| \leq 1$ e $\left|\lambda_{2}\right| \geq 1$ e os pontos fixos serão hiperbólicos, isto é, os pontos fixos serão instáveis. No caso especial em que $\lambda_{1}=\lambda_{2}= \pm 1$, os pontos fixos serão parabólicos e pontos fixos parabólicos geralmente são instáveis [40].

Logo, a estabilidade dos pontos fixos é alterada quando os autovalores $\lambda_{1,2}$ passam de imaginário para real. A partir da equação (35) observa-se que essa transição acontece para 


$$
\begin{aligned}
& \left(\frac{\pi\left(\varepsilon k^{2}\right)_{\mathrm{bdp}}}{2}-1-\frac{2 \pi^{3}\left(\varepsilon k^{2}\right)_{\mathrm{bdp}}}{T^{2}}\right)^{2}-1=0, \\
& \frac{\pi\left(\varepsilon k^{2}\right)_{\mathrm{bdp}}}{2}-1-\frac{2 \pi^{3}\left(\varepsilon k^{2}\right)_{\mathrm{bdp}}}{T^{2}}= \pm 1, \\
& \left(\varepsilon k^{2}\right)_{\mathrm{bdp}}\left(\frac{\pi}{2}-\frac{2 \pi^{3}}{T^{2}}\right)=2 \text { ou } 0
\end{aligned}
$$

onde $\left(\varepsilon k^{2}\right)_{\text {bdp }}$ indica os parâmetros da onda eletrostática para os quais a ressonância $(1,1)$ sofre uma bifurcação com dobramento de período. Como $\varepsilon$ e $k$ são parâmetros da onda, eles não podem ser nulos. Portanto, a expressão (36) torna-se [16]

$$
\left(\varepsilon k^{2}\right)_{\mathrm{bdp}}=\frac{4 T^{2}}{\pi\left(T^{2}-4 \pi^{2}\right)} .
$$

Para $\left(\varepsilon k^{2}\right)_{\text {bdp }}<4 T^{2}\left[\pi\left(T^{2}-4 \pi^{2}\right)\right]^{-1}$, os pontos fixos $I^{*}=I_{1,1}=T^{2} / 8 \pi^{2}-0,5$ e $\theta^{*}=0 ; \pi$ são elípticos. Para $\left(\varepsilon k^{2}\right)_{\text {bdp }}>4 T^{2}\left[\pi\left(T^{2}-4 \pi^{2}\right)\right]^{-1}$, esses pontos fixos são hiperbólicos. Portanto, a expressão (37) fornece os parâmetros da onda eletrostática para os quais os pontos elípticos da ressonância $(1,1)$ perdem estabilidade, tornando-se hiperbólicos.

Os cálculos feitos nesta seção se referem aos pontos fixos da ressonância $(1,1)$ localizados em $I^{*}=\mathrm{I}_{1,1}=T^{2} / 8 \pi^{2}-0,5$ e $\theta^{*}=0 ; \pi$. Como discutido na Seção 2.3, a ressonância principal só está presente no espaço de fases quando $T>2 \pi$. Isso indica que $\left(\varepsilon k^{2}\right)_{\mathrm{bdp}}>0$ na equação (37) e, portanto, essa expressão é válida sempre.

Para $T=2 \pi(1+1 / 15)$ como nas figuras dos Capítulos 2 e $4,\left(\varepsilon k^{2}\right)_{\text {bdp }} \cong$ 10,514. Além disso, essas figuras foram geradas para $k=2 \mathrm{e}$, portanto, $\varepsilon_{\text {bdp }} \cong 2,629$. Por esse motivo, na Figura 14 foram escolhidos os parâmetros $\varepsilon=2,500$ (antes da bifurcação) e $\varepsilon=2,750$ (depois da bifurcação).

As equações (31) e (37) estão representadas na Figura 15. A curva tracejada corresponde aos parâmetros da onda eletrostática para os quais os pontos fixos da ressonância $(1,1)$ localizados em $\theta=0 ; \pi$ sofrem uma bifurcação com dobramento de período (equação (37)). Para valores abaixo da curva, os pontos fixos são elípticos (estáveis) e para valores acima da curva, os pontos fixos são hiperbólicos (instáveis). A curva sólida da Figura 15 representa os valores mínimos dos parâmetros da onda para os quais os pontos hiperbólicos da ressonância $(1,1)$ estão localizados sobre o eixo $I=0$ (equação (31)). Para valores abaixo da 
curva, os pontos hiperbólicos estão acima do eixo $I=0$ e para valores acima da curva, os pontos hiperbólicos estão localizados sobre o eixo $I=0$.

No ponto de encontro das duas curvas da Figura $15, T \cong 2,474 \pi, \varepsilon k^{2} \cong$ 3,678 e os pontos hiperbólicos da ressonância $(1,1)$ atingem o eixo $I=0$ simultaneamente com a perda de estabilidade dos pontos elípticos. Logo, só é possível acelerar a partícula de forma regular nas ilhas da ressonância $(1,1)$ quando o período da onda eletrostática for menor do que $2,474 \pi$. Além disso, o valor de $\varepsilon k^{2}$ deve estar na região sombreada da Figura 15. Para $T>2,474 \pi$, os pontos elípticos em $\theta=0 ; \pi$ perdem estabilidade antes dos pontos hiperbólicos atingirem o eixo $I=0$ e a aceleração da partícula não será máxima.

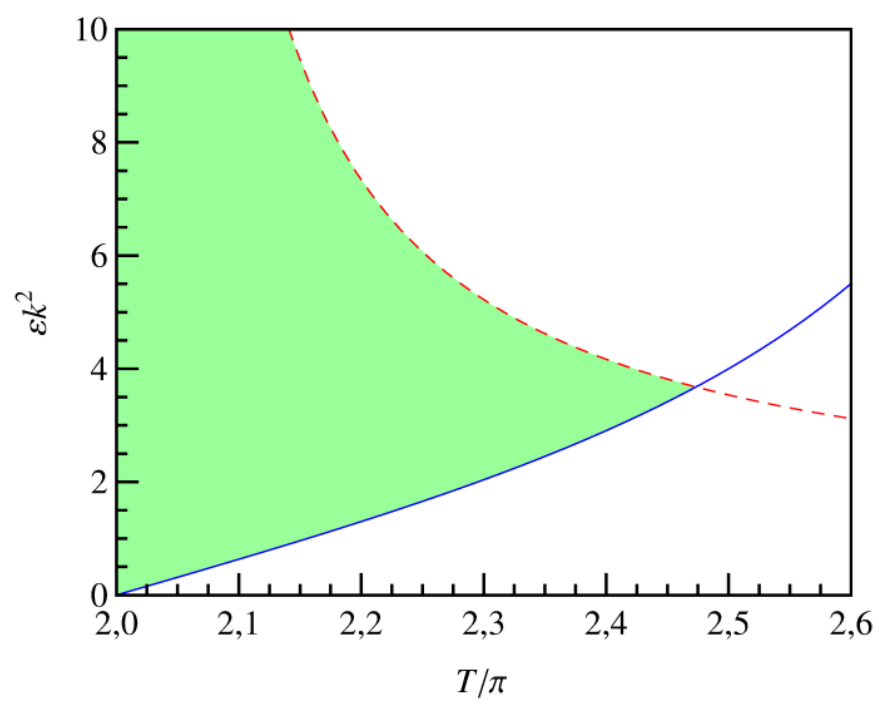

Figura 15: A curva tracejada corresponde aos parâmetros da onda para os quais os pontos elípticos da ressonância $(1,1)$ localizados em $\theta=0 ; \pi$ sofrem uma bifurcação com dobramento de período. A curva sólida representa os valores mínimos dos parâmetros da onda para os quais os pontos hiperbólicos da ressonância $(1,1)$ estão localizados sobre o eixo $I=0$. A região sombreada indica o intervalo de parâmetros para os quais é possível acelerar a partícula de forma regular nas ilhas da ressonância $(1,1)$.

Fonte: Referência [16].

\subsection{CONCLUSÃO}

Ao longo deste capítulo foram analisadas as principais características da ressonância $(1,1)$ do sistema. Entre essas características pode-se citar o deslocamento dos pontos hiperbólicos provocado pelo aumento dos parâmetros da onda eletrostática. Quando os parâmetros da onda atingem valores adequados, os pontos hiperbólicos são deslocados para o 
eixo da variável de ação $I=0$, contribuindo para a aceleração regular de partículas a partir de baixas energias iniciais [16].

Entretanto, existe um limite natural para o processo de aceleração regular que ocorre quando os pontos elípticos da ressonância principal perdem estabilidade e consequentemente as ilhas de ressonância são destruídas. Analisando a estabilidade dos pontos fixos foi possível determinar uma expressão analítica que fornece os parâmetros da onda para os quais os pontos elípticos perdem estabilidade.

Comparando a expressão que determina o deslocamento dos pontos hiperbólicos para o eixo da variável de ação $I=0$ e a expressão que determina a bifurcação dos pontos elípticos, encontra-se o intervalo de parâmetros da onda eletrostática para os quais é possível acelerar a partícula de forma regular nas ilhas da ressonância $(1,1)$.

É importante ressaltar que as ilhas regulares da ressonância principal e o fenômeno de bifurcação dos pontos elípticos só estão presentes no sistema quando a massa da partícula é corrigida através da expressão relativística [16]. Se os efeitos relativísticos não forem considerados, as ilhas de ressonância são substituídas por toros invariantes [70]. 


\section{CONTROLE GLOBAL DO CAOS}

A Ref. [33] apresenta um método de controle do caos para sistemas Hamiltonianos quase integráveis. O objetivo desse método é determinar um termo de controle de baixa amplitude que seja capaz de tornar o sistema controlado mais regular do que o sistema original. Neste capítulo, o método de controle desenvolvido na Ref.[33] é descrito de forma resumida e ele é aplicado ao sistema em estudo. Os espaços de fases do sistema original e do sistema controlado são comparados e observa-se que o termo de controle restaura diversos toros invariantes no sistema controlado [17]. Esses toros confinam as trajetórias caóticas e impedem que elas ocupem grandes áreas no espaço de fases.

Por ser mais regular, verifica-se que no sistema controlado ocorre uma melhora do processo de aceleração regular de partículas nas ilhas de ressonância [17]. Além disso, o sistema controlado apresenta a mesma estrutura de ilhas primárias do sistema original. Essa é uma das características mais importantes do método de controle utilizado: ele controla o caos no sistema sem alterar suas estruturas principais.

Os resultados apresentados neste capítulo sobre o controle do caos no sistema em estudo foram publicados na Ref. [17] e apresentados em conferências internacionais.

\subsection{MÉTODO DE CONTROLE}

Nesta seção, é apresentado de forma resumida o método de controle do caos proposto na Ref. [33]. Esse método é baseado em teoria de perturbação e álgebra de Lie e ele se destina a sistemas conservativos que podem ser descritos por uma Hamiltoniana quase integrável na forma $H=H_{0}+\varepsilon V$, onde $H_{0}$ é integrável e $\varepsilon V$ é uma perturbação pequena comparada a $H_{0} \operatorname{com} \varepsilon<<1$. Para $\varepsilon=0$, a dinâmica é integrável e o espaço de fases apresenta apenas toros invariantes. À medida que $\varepsilon$ aumenta, as trajetórias são alteradas e o sistema se torna cada vez mais caótico.

O objetivo do método apresentado na Ref. [33] é determinar um termo de controle $f(\varepsilon V)$, de tal forma que a Hamiltoniana controlada $H_{f}=H_{0}+\varepsilon V+f(\varepsilon V)$ seja integrável ou apresente um comportamento mais regular do que o sistema original. Nesse 
caso, $f=-\varepsilon V$ seria uma possível solução, pois com esse termo de controle, a nova Hamiltoniana seria integrável. Entretanto, $f=-\varepsilon V$ é da mesma ordem da perturbação originalmente aplicada ao sistema. Por razões energéticas, o termo de controle deve ser muito menor do que $\varepsilon V$, por exemplo, se $\varepsilon V$ é de ordem $\varepsilon$, então $f(\varepsilon V)$ deve ser de ordem $\varepsilon^{2}$. Dessa forma, o método em estudo procura encontrar um termo de controle de baixa amplitude que modifique muito pouco o sistema, mas seja capaz de reduzir as regiões de comportamento caótico no espaço de fases.

O termo de controle pode ser global e agir em todo o espaço de fases [34, 37, 38 ], como é o caso de $f(\varepsilon V)$, ou ele pode ser um termo de controle local que atua em uma região específica do espaço de fases $[25,39,71]$. Neste capítulo será descrito apenas o procedimento para determinação de termos de controle globais, uma vez que esse procedimento também é utilizado na dedução de termos de controle locais, como pode ser visto nas Refs. [39, 71].

Seja $A$ a álgebra de Lie de funções reais de classe $\mathbb{C}^{\infty}$ definidas no espaço de fases. Considere um elemento $H \in A$, onde $H$ é uma função Hamiltoniana. $\mathrm{O}$ operador linear associado a $H$ é escrito como $\{H\}$ e ele atua em $A$ de tal forma que

$$
\{H\} H^{\prime}=\left\{H, H^{\prime}\right\}
$$

para qualquer $H^{\prime} \in A$, onde $\{$,$\} é o parênteses de Poisson. Seja H_{0} \in A$ uma Hamiltoniana integrável escrita em termos das variáveis de ângulo e ação $(\vec{I}, \vec{\theta}) \in B \times T^{n}$, onde $B$ é um domínio de $\mathbb{R}^{n}, T^{n}$ é um toro n-dimensional e $n$ é o número de graus de liberdade. Usando variáveis de ângulo e ação, o parênteses de Poisson de duas Hamiltonianas é dado por

$$
\left\{H, H^{\prime}\right\}=\sum_{j=1}^{n}\left(\frac{\partial H}{\partial I_{j}} \frac{\partial H^{\prime}}{\partial \theta_{j}}-\frac{\partial H}{\partial \theta_{j}} \frac{\partial H^{\prime}}{\partial I_{j}}\right) .
$$

Considere uma função $V(\vec{I}, \vec{\theta}) \in A$ expandida em série de Fourier como

$$
V(\vec{I}, \vec{\theta})=\sum_{\vec{k} \in \mathbb{Z}^{n}} V_{\vec{k}}(\vec{I}) \mathrm{e}^{\mathrm{i} \vec{k} \cdot \vec{\theta}}
$$

e a ação do operador $\left\{H_{0}\right\}$ em $V$ como

$$
\left\{H_{0}\right\} V(\vec{I}, \vec{\theta})=\sum_{\vec{k} \in \mathbb{Z}^{n}} \mathrm{i} \vec{\omega}_{0}(\vec{I}) \cdot \vec{k} V_{\vec{k}}(\vec{I}) \mathrm{e}^{\mathrm{i} \vec{k} \cdot \vec{\theta}}
$$


onde $\vec{\omega}_{0}(\vec{I})=\partial H_{0} / \partial \vec{I}$ é a frequência não perturbada associada ao sistema descrito pela Hamiltoniana integrável $H_{0}$.

Como o operador $\left\{H_{0}\right\}$ não possui um inverso estrito [33], defini-se um pseudoinverso de $\left\{H_{0}\right\}$ como um operador linear $\Gamma$ que atua em $A$

$$
\left\{H_{0}\right\}^{2} \Gamma=\left\{H_{0}\right\}
$$

O operador $\Gamma$ é escolhido de tal forma que ele comute com $\left\{H_{0}\right\}$ e sua ação em $V$ seja dada por

$$
\Gamma V(\vec{I}, \vec{\theta})=\sum_{\substack{\vec{k} \in \mathbb{Z}^{n} \\ \omega_{0}(\vec{I}) \cdot \vec{k} \neq 0}} \frac{V_{\vec{k}}(\vec{I})}{\mathrm{i} \vec{\omega}_{0}(\vec{I}) \cdot \vec{k}} \mathrm{e}^{\mathrm{i} \vec{k} \cdot \vec{\theta}}
$$

Além dos operadores $\left\{H_{0}\right\}$ e $\Gamma$, outros dois operadores são construídos, $N$ e $R \cdot R V$ é definido como a parte ressonante de $V$ e $N V$ é sua parte não ressonante, como segue

$$
\begin{aligned}
& R V(\vec{I}, \vec{\theta})=\sum_{\substack{\vec{k} \in \mathbb{Z}^{n} \\
\vec{\omega}_{0}(\vec{I}) \cdot \vec{k}=0}} V_{\vec{k}}(\vec{I}) \mathrm{e}^{\mathrm{i} \vec{k} \cdot \vec{\theta}}, \\
& N V(\vec{I}, \vec{\theta})=\sum_{\substack{\vec{k} \in \mathbb{Z}^{n} \\
\bar{\omega}_{0}(\vec{I}) \cdot \vec{k} \neq 0}} V_{\vec{k}}(\vec{I}) \mathrm{e}^{\mathrm{i} \vec{k} \cdot \vec{\theta}} .
\end{aligned}
$$

Finalmente, a função $f(V)$ que torna o sistema controlado mais regular do que o sistema original é definida como

$$
f(V)=\sum_{m=1}^{\infty} \frac{(-1)^{m}}{(m+1) !}\{\Gamma V\}^{m}(m R+1) V
$$

e é possível provar que a expressão (38) é de fato um termo de controle, como pode ser visto na Ref. [33]. A partir da expressão (38) observa-se que se $V$ é de ordem $\varepsilon$, então $f(V)$ possui um termo dominante de ordem $\varepsilon^{2}$. Logo, $f(V)$ é muito menor do que $V$, uma vez que $\varepsilon<<1$. 


\subsection{APLICAÇÃO DO MÉTODO DE CONTROLE}

A partir da expressão (38), calcula-se o termo de controle para o sistema descrito pela Hamiltoniana (7). Entretanto, essa Hamiltoniana possui 1,5 graus de liberdade, pois ela é função das coordenadas $(I, \theta)$ e do tempo $t$. Por esse motivo, é necessário reescrever a Hamiltoniana (7) como uma Hamiltoniana autônoma com 2 graus de liberdade. Isso é feito considerando o tempo como uma variável de ângulo adicional e a energia $E$ será a ação conjugada a $t$. A nova Hamiltoniana com 2 graus de liberdade é dada por [17]

$$
\begin{aligned}
& H(I, E, \theta, t)=H_{0}(I, E)+\varepsilon V(I, \theta, t), \\
& H(I, E, \theta, t)=E+\sqrt{1+2 I}+\frac{\varepsilon}{2} \cos (k \sqrt{2 I} \operatorname{sen} \theta) \sum_{n=-\infty}^{+\infty} \delta(t-n T) .
\end{aligned}
$$

Para que os parênteses de Poisson presentes na teoria de controle possam ser calculados corretamente, a soma periódica de funções delta na Hamiltoniana (39) será reescrita como uma série de Fourier utilizando a expressão (12)

$$
H(I, E, \theta, t)=E+\sqrt{1+2 I}+\frac{\varepsilon}{2 T} \cos (k \sqrt{2 I} \operatorname{sen} \theta) \sum_{n=-\infty}^{+\infty} \cos \left(\frac{2 \pi n t}{T}\right)
$$

Os operadores $\left\{H_{0}\right\}, \Gamma, R$ e $N$ atuam sobre a perturbação $\varepsilon V$ dada em (40) como segue [17]

$$
\begin{aligned}
&\left\{H_{0}\right\}(\varepsilon V)=-\frac{2 \pi \varepsilon}{2 T^{2}} \cos (k \sqrt{2 I} \operatorname{sen} \theta) \sum_{n=-\infty}^{+\infty} n \operatorname{sen}\left(\frac{2 \pi n t}{T}\right) \\
&-\frac{\varepsilon k \sqrt{2 I} \cos \theta}{2 T \sqrt{1+2 I}} \operatorname{sen}(k \sqrt{2 I} \operatorname{sen} \theta) \sum_{n=-\infty}^{+\infty} \cos \left(\frac{2 \pi n t}{T}\right), \\
& \Gamma(\varepsilon V)=-\frac{\varepsilon}{4 \pi} \cos (k \sqrt{2 I} \operatorname{sen} \theta) \sum_{n=-\infty}^{+\infty} \frac{1}{n} \operatorname{sen}\left(\frac{2 \pi n t}{T}\right) \\
& \quad-\frac{\varepsilon \sqrt{1+2 I}}{2 T k \sqrt{2 I} \cos \theta} \operatorname{sen}(k \sqrt{2 I} \operatorname{sen} \theta) \sum_{n=-\infty}^{+\infty} \cos \left(\frac{2 \pi n t}{T}\right) \\
&\left(I \neq 0 \text { e } \theta \neq \frac{\pi / 2,3 \pi / 2 \operatorname{para} 0 \leq \theta<2 \pi),}{n \neq 0},\right. \\
& N(\varepsilon V)=\frac{\varepsilon}{2 T} \cos (k \sqrt{2 I} \operatorname{sen} \theta) \sum_{n=-\infty}^{+\infty} \cos \left(\frac{2 \pi n t}{T}\right),
\end{aligned}
$$




$$
R(\varepsilon V)=0
$$

Como a Hamiltoniana (40) é dada na forma $H=H_{0}+\varepsilon V$, onde $H_{0}$ é integrável e $\varepsilon V$ é uma perturbação pequena em comparação a $H_{0}$, é possível obter um termo de controle $f(\varepsilon V)$ que torne o sistema mais regular. A partir da expressão (38), observa-se que $f$ é dado por uma soma infinita de termos. Entretanto, a literatura mostra que é possível obter bons resultados mantendo apenas um ou dois termos em $f[34,37,38]$, o que prova o caráter robusto da teoria de controle. Além disso, truncar a função $f$ é conveniente, e muitas vezes necessário, pois o termo de controle deve ser o mais simples possível para que seja viável implementá-lo experimentalmente.

Utilizando as expressões (41) - (44) e mantendo apenas os termos até $m=1$ na equação (38), o termo de controle para a Hamiltoniana (40) é dado por [17]

$$
\begin{aligned}
& f(\varepsilon V) \cong-\frac{1}{2}\{\Gamma(\varepsilon V)\}(\varepsilon V), \\
& f(\varepsilon V) \cong \frac{\varepsilon^{2}}{8 T^{2}} \operatorname{sen}^{2}(k \sqrt{2 I} \operatorname{sen} \theta)\left[\frac{\sqrt{1+2 I}}{2 I}+\frac{\sqrt{1+2 I}}{2 I} \operatorname{tg}^{2} \theta-\frac{1}{\sqrt{1+2 I}}\right]\left[\sum_{n=-\infty}^{+\infty} \cos \left(\frac{2 \pi n t}{T}\right)\right]^{2}, \\
& f(\varepsilon V) \cong \frac{\varepsilon^{2}}{16}[1-\cos (2 k \sqrt{2 I} \operatorname{sen} \theta)]\left[\frac{\sqrt{1+2 I}}{2 I}+\frac{\sqrt{1+2 I}}{2 I} \operatorname{tg}^{2} \theta-\frac{1}{\sqrt{1+2 I}}\right] \sum_{n=-\infty}^{+\infty} \delta(t-n T), \\
& (I \neq 0 \text { e } \theta \neq \pi / 2,3 \pi / 2 \text { para } 0 \leq \theta<2 \pi),
\end{aligned}
$$

onde foi utilizada a expressão (12) na última passagem. A partir da expressão (45), observa-se que os termos proporcionais a $\left(\partial H_{0} / \partial E\right)(\partial V / \partial t)$ se cancelam. Entretanto, essa é uma característica particular da Hamiltoniana (40). O cancelamento dos termos proporcionais a $\left(\partial H_{0} / \partial E\right)(\partial V / \partial t)$ não ocorre em todos os sistemas, como pode ser visto, por exemplo, nas Refs. [38, 71], onde esses termos não se cancelam aos pares.

Para evitar indeterminações e um crescimento ilimitado da função $f(\varepsilon V)$ em regiões específicas do espaço de fases, o termo de controle (45) será truncado e apenas o último termo dessa expressão será mantido. Além disso, o termo $(1+2 I)^{-1 / 2}$ será expandido em série de Taylor ao redor de $I_{0}$ como

$$
\frac{1}{\sqrt{1+2 I}}=\frac{1}{\sqrt{1+2 I_{0}}}-\frac{I-I_{0}}{\left(1+2 I_{0}\right)^{3 / 2}}+\frac{3\left(I-I_{0}\right)^{2}}{2\left(1+2 I_{0}\right)^{5 / 2}}-\ldots=C+O(I)
$$

onde $C$ representa todos os termos constantes da expansão e $O(I)$ representa todos os termos 
proporcionais a $I^{j}$ com $j=1,2,3, \ldots$.

Truncando a expressão (45) como mencionado anteriormente e substituindo a expansão (46), o termo de controle resultante é dado por

$$
f(\varepsilon V)=\frac{\varepsilon^{2}}{16}\{C \cos (2 k \sqrt{2 I} \operatorname{sen} \theta)+O(I)[\cos (2 k \sqrt{2 I} \operatorname{sen} \theta)-1]-C\} \sum_{n=-\infty}^{+\infty} \delta(t-n T)
$$

O primeiro termo da equação (47) é semelhante ao termo perturbativo da Hamiltoniana (7), tendo a mesma interpretação física, isto é, o primeiro termo da equação (47) também é uma onda eletrostática e estacionária. Por esse motivo, o termo de controle $f(\varepsilon V)$ será truncado mais uma vez e apenas o primeiro termo da expressão (47) será mantido [17]

$$
f(\varepsilon V)=\varepsilon^{2} a \cos (2 k \sqrt{2 I} \operatorname{sen} \theta+\gamma) \sum_{n=-\infty}^{+\infty} \delta(t-n T)
$$

Como o termo de controle (48) não possui todos os termos da série infinita dada pela expressão (38), a amplitude e a fase da onda eletrostática serão consideradas como dois parâmetros que podem variar livremente. Simulações numéricas mostram que a eficiência do termo de controle é máxima para $a=1 / 8$ e $\gamma=\pi$ [17]. Logo, o termo de controle final que será adicionado à Hamiltoniana (7) é dado por

$$
f(\varepsilon V)=\frac{\varepsilon^{2}}{8} \cos (2 k \sqrt{2 I} \operatorname{sen} \theta+\pi) \sum_{n=-\infty}^{+\infty} \delta(t-n T)
$$

e observa-se que $f$ corresponde simplesmente a uma onda eletrostática e estacionária dada na forma de pulsos periódicos com vetor de onda $2 \vec{k}$, período $T$, amplitude $\varepsilon^{2} / 8$ e fase $\pi$ se propagando ao longo do eixo $x$.

\subsection{SISTEMA CONTROLADO}

A Hamiltoniana do sistema descrito na Seção 2.1 com a adição do termo de controle (49) é dada por

$$
H(I, \theta, t)=\sqrt{1+2 I}+\left[\frac{\varepsilon}{2} \cos (k \sqrt{2 I} \operatorname{sen} \theta)+\frac{\varepsilon^{2}}{8} \cos (2 k \sqrt{2 I} \operatorname{sen} \theta+\pi)\right] \sum_{n=-\infty}^{+\infty} \delta(t-n T) .
$$


Seguindo novamente o procedimento desenvolvido na Seção 2.2, obtém-se um mapa exato e explícito que relaciona as variáveis $I$ e $\theta$ nos instantes de tempo $t=n T$ e $t=(n+1) T$ [17]

$$
\left.\begin{array}{rl}
I_{n+1}= & \frac{1}{2}\left\{2 I_{n} \operatorname{sen}^{2} \theta_{n}+\left[\sqrt{2 I_{n}} \cos \theta_{n}+\frac{\varepsilon k}{2} \operatorname{sen}\left(k \sqrt{2 I_{n}} \operatorname{sen} \theta_{n}\right)\right.\right. \\
& \left.\left.+\frac{\varepsilon^{2} k}{4} \operatorname{sen}\left(2 k \sqrt{2 I_{n}} \operatorname{sen} \theta_{n}+\pi\right)\right]^{2}\right\}, \\
\theta_{n+1}= & \operatorname{arctg}\left[\frac{4 \sqrt{2 I_{n}} \operatorname{sen} \theta_{n}}{4 \sqrt{2 I_{n}}} \cos \theta_{n}+2 \varepsilon k \operatorname{sen}\left(k \sqrt{2 I_{n}} \operatorname{sen} \theta_{n}\right)+\varepsilon^{2} k \operatorname{sen}\left(2 k \sqrt{2 I_{n}} \operatorname{sen} \theta_{n}+\pi\right)\right.
\end{array}\right]
$$

A Figura 16 contém os espaços de fases construídos a partir dos mapas (8) e (51) para $T=2 \pi(1+1 / 15), k=2$ e $\varepsilon=0,2$. A Figura 16(a) mostra o sistema sem o termo de controle e observa-se que as regiões de comportamento caótico preenchem grande parte do espaço de fases. Apenas a região de baixa ação $I$ e algumas ilhas de ressonância permanecem regulares. A Figura 16(b) mostra o sistema com a adição do termo de controle (49). Ao contrário da Figura 16(a), o espaço de fases da Figura 16(b) apresenta órbitas regulares em quase toda sua extensão. Regiões de comportamento caótico podem ser vistas apenas ao redor das ilhas de ressonância por causa dos pontos hiperbólicos que separam as ilhas.

Na Figura 16(a), as trajetórias caóticas são mais densas na região $0,3<I<1,3$, indicando um aprisionamento das trajetórias ao redor das ilhas de ressonância, como discutido na Seção 2.3. Contudo, após um número suficiente de iterações, as trajetórias caóticas escapam dessa região, atingindo valores de ação arbitrariamente elevados. Por esse motivo, pode-se dizer que para $I>0,3$ o caos é global e restam apenas algumas ilhas de comportamento regular imersas no mar de caos.

Por outro lado, na Figura 16(b) as trajetórias caóticas estão confinadas em pequenas regiões ao redor dos pontos hiperbólicos das ressonâncias e na separatriz das ilhas. A adição do termo de controle ao sistema cria diversos toros invariantes que separam as regiões de ressonância e confinam as trajetórias caóticas no espaço de fases. A Figura 17 mostra uma ampliação da Figura 16 (b) na região $0,18<I<0,90$ e é possível observar com clareza os toros invariantes presentes entre as cadeias de ilhas no espaço de fases.

Para $\varepsilon=0,2$ como na Figura 16, a amplitude do termo de controle $f$ é apenas 
$5 \%$ da amplitude de $\varepsilon V$, isto é, para gerar a onda eletrostática descrita por $f$, gasta-se apenas $5 \%$ da energia utilizada para produzir a onda eletrostática $\varepsilon V$. Esta é uma das características mais importantes do procedimento utilizado na determinação do termo de controle: a função $f(\varepsilon V)$ é capaz de controlar o caos no sistema com um baixo custo energético. Essa característica e a forma simples da função $f$ tornam possível a utilização do termo de controle em experimentos, como realizado na Ref. [34] e discutido na Seção 5.5.

(a) Sem controle do caos

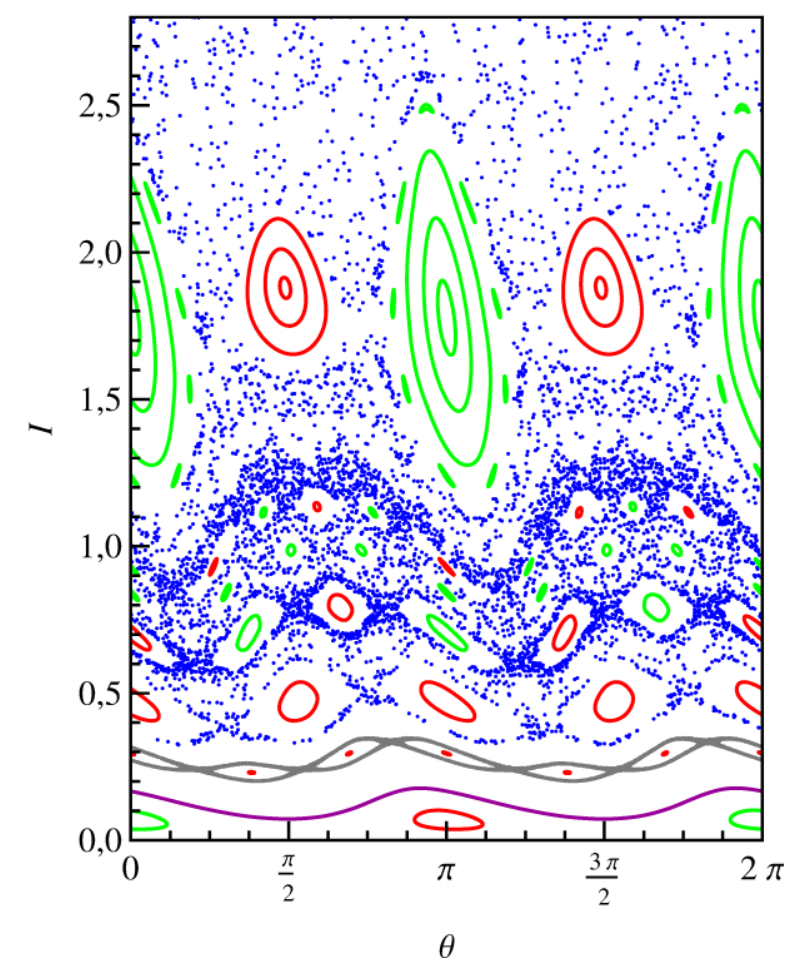

(b) Com controle do caos

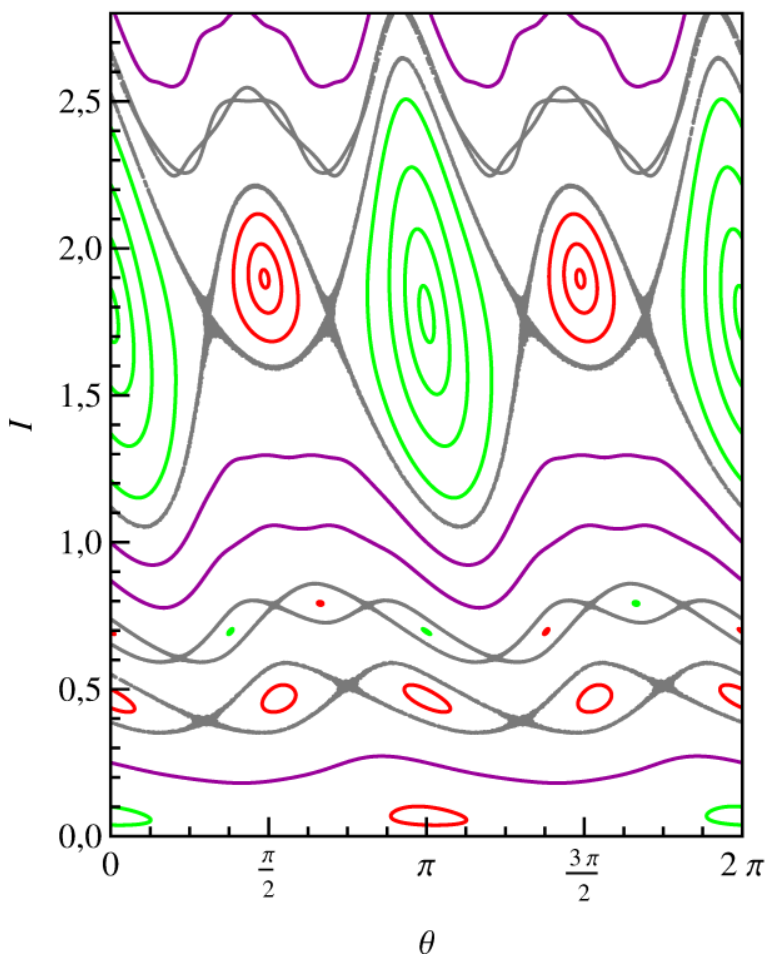

Figura 16: Espaço de fases do sistema a) sem o termo de controle e b) com a adição do termo de controle. Parâmetros utilizados: $T=2 \pi(1+1 / 15), k=2$ e $\varepsilon=0,200$.

Fonte: Referência [17].

Além disso, o sistema controlado possui as mesmas ressonâncias primárias do sistema original [17], como, por exemplo, as ressonâncias $(1,1),(4,3),(3,2)$ e $(2,1)$ localizadas respectivamente ao redor de $I_{1,1} \cong 0,069 ; I_{4,3} \cong 0,511 ; I_{3,2} \cong 0,780$ e $I_{2,1} \cong 1,776$. Isso significa que a adição de um termo de controle adequado e com baixa amplitude não altera as estruturas principais do sistema, embora ele reduza drasticamente as regiões de comportamento caótico no espaço de fases.

Seguindo o procedimento descrito na Seção 3.2, verifica-se que as ressonâncias primárias da Hamiltoniana (50) também são dadas pela expressão (18). Além disso, 
expandindo o mapa controlado (51) em série de Taylor até primeira ordem em $\varepsilon$, obtém-se os mesmos resultados da Seção 3.3. Portanto, até primeira ordem em $\varepsilon$, os pontos periódicos do mapa (8) que descreve o sistema original e do mapa (51) que descreve o sistema controlado estão localizados na mesma posição $(I, \theta)$ no espaço de fases, como pode ser visto comparando os dois quadros da Figura 16.

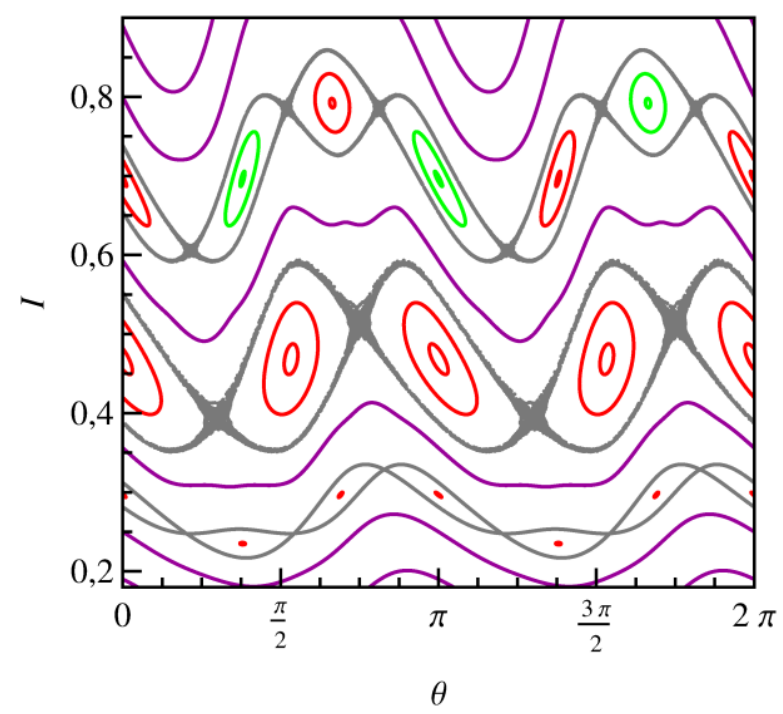

Figura 17: Toros invariantes separando as regiões de ressonância no espaço de fases do sistema controlado. Parâmetros utilizados: $T=2 \pi(1+1 / 15), k=2$ e $\varepsilon=0,200$.

Para tentar entender como acontece o acoplamento das duas ondas eletrostáticas e o papel desempenhado por $f(\varepsilon V)$ no espaço de fases da Figura 16(b), será estudada a seguir uma Hamiltoniana perturbada apenas pelo termo de controle. A Figura 18 mostra o espaço de fases do sistema descrito pela Hamiltoniana (52) para os mesmos parâmetros utilizados na Figura 16:

$$
H=H_{0}+f=\sqrt{1+2 I}+\frac{\varepsilon^{2}}{8} \cos (2 k \sqrt{2 I} \operatorname{sen} \theta+\pi) \sum_{n=-\infty}^{+\infty} \delta(t-n T)
$$

Quase todas as trajetórias presentes na Figura 18 são regulares. Apenas a separatriz das ilhas e órbitas muito próximas dos pontos hiperbólicos são caóticas. Além disso, a região ocupada pelas trajetórias caóticas no espaço de fases é muito pequena.

Mais uma vez observa-se que a Hamiltoniana (52) apresenta as mesmas ressonâncias primárias do sistema original. Por exemplo, as ressonâncias $(1,1),(2,1),(3,2)$ e $(4,3)$ estão localizadas na mesma posição em $I$ nas Figuras 16(a) e 18. Essa é uma característica extremamente importante do método de controle utilizado. Seguindo o 
procedimento descrito na Seção 3.2, observa-se que a posição em $I$ das ressonâncias primárias da Hamiltoniana (52) também é dada pela expressão (18). Entretanto, a posição das ilhas de ressonância com relação à variável $\theta$ não é a mesma nas Figuras 16(a) e 18, assim como a estabilidade de alguns pontos periódicos e o número de cadeias de ilhas presentes nas regiões de ressonância do sistema.

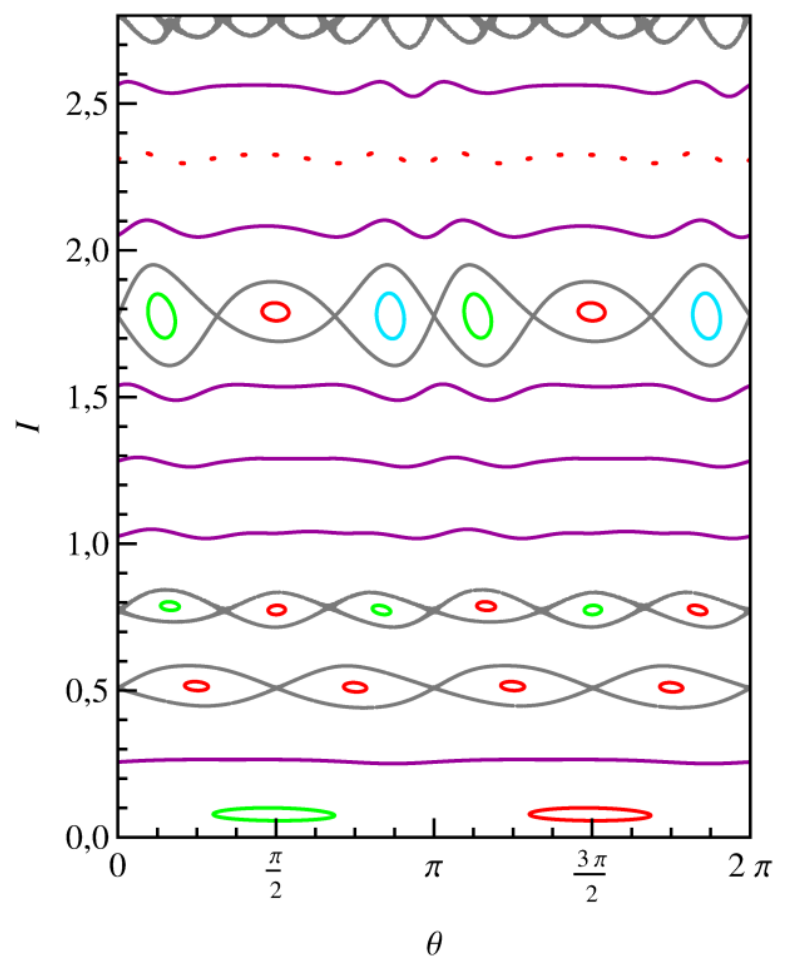

Figura 18: Espaço de fases do sistema perturbado apenas pelo termo de controle. Parâmetros utilizados: $T=2 \pi(1+1 / 15), k=2$ e $\varepsilon=0,200$.

Fonte: Referência [17].

A partir dos procedimentos descritos nas Seções 3.3 e 3.4, é possível determinar o valor de $\theta$ nos pontos periódicos e o número de cadeias presentes em cada ressonância. Entretanto, é preciso notar que a Hamiltoniana (52) não possui termos lineares em $\varepsilon$. Por esse motivo, o sistema perturbado apenas pelo termo de controle deve ser expandido até segunda ordem em $\varepsilon$ para que sejam obtidos os resultados desejados.

Comparando os espaços de fases das Figuras 16 e 18, observa-se que o sistema perturbado pelas duas ondas eletrostáticas $\varepsilon V+f$ apresenta um comportamento que é uma mistura dos comportamentos individuais dos sistemas descritos pelas Hamiltonianas (7) e (52). A amplitude de $f$ é muito menor do que a amplitude de $\varepsilon V$, assim como o tamanho das ilhas criadas por $f$ no espaço de fases. Portanto, a estrutura de ilhas da Figura 16(b) é a 
mesma da Figura 16(a) que corresponde ao sistema sem o termo de controle.

Na Figura 16(a), quase toda a região externa às principais ilhas do sistema é preenchida por trajetórias caóticas, enquanto na Figura 18 todas as trajetórias são regulares nessa região. Na Figura 16(b), a região exterior às ilhas é regularizada e apresenta diversos toros invariantes. A partir desses resultados conclui-se que embora o termo de controle seja muito menor do que $\varepsilon V$, o comportamento associado a $f$ é predominante na região considerada [17]. A adição do termo de controle ao sistema cria toros invariantes em todo o espaço de fases que previnem o surgimento de trajetórias caóticas e tornam o sistema controlado mais regular do que o sistema original.

\subsection{ACELERAÇÃO REGULAR DE PARTÍCULAS NO SISTEMA CONTROLADO}

No Capítulo 4 e na Ref. [16] foi analisada a aceleração regular de partículas na ressonância principal do sistema que está localizada na região de baixa ação $I<0,20$. Entretanto, também é possível acelerar partículas de forma regular em regiões de ação mais elevada, como pode ser visto na Figura 16 para $1,00<I<2,70$. Considerando as ilhas centradas em $\theta=0 ; \pi$, observa-se que o valor da ação aumenta quando a onda eletrostática transfere energia para a partícula. Na Figura 16(a), as trajetórias mais externas das ilhas centradas em $I_{2,1} \cong 1,776$ e $\theta=0 ; \pi$ foram destruídas pelo caos e apenas as trajetórias mais internas sobrevivem. Na Figura 16(b), a adição do termo de controle ao sistema recupera as trajetórias mais externas dessas ilhas, melhorando o processo de aceleração regular.

Numericamente é possível determinar a largura máxima das ilhas de ressonância centradas em $I_{2,1} \cong 1,776$ e $\theta=0 ; \pi$, assim como a energia, o momento e a velocidade adimensionais mínimos e máximos da partícula nas ilhas, como mostra a Tabela 2. A energia adimensional da partícula é dada pela expressão $E \approx \sqrt{1+2 I}$, o momento é obtido a partir da transformação canônica $p_{x}=\sqrt{2 I} \cos \theta$, e a velocidade é dada por $v_{x}=p_{x}\left(1+p_{x}^{2}\right)^{-1 / 2}$, como visto na Seção 4.2 e na Ref. [16].

A partir dos dados da Tabela 2, verifica-se que para o sistema sem o termo de controle, a energia adimensional mínima da partícula é $E_{\text {mín }} \cong 1,872$ e sua energia máxima é $E_{\text {máx }} \cong 2,398$ [17]. Com a adição do termo de controle, a energia mínima da particular nas 
ilhas de ressonância é $E_{\text {mín }} \cong 1,766$ e sua energia máxima é $E_{\text {máx }} \cong 2,505$. Portanto, com a adição do termo de controle ao sistema, a energia mínima da partícula é 5,35\% menor do que sua energia mínima no sistema original. Por outro lado, a energia máxima da partícula é 4,17\% maior no sistema controlado. Isso significa que, no sistema controlado, a energia inicial da partícula pode ser menor do que no sistema original e ainda assim a partícula é mais acelerada pela onda eletrostática, pois sua energia final é maior.

Tabela 2 - Energia, módulo do momento e módulo da velocidade adimensionais mínimos e máximos da partícula nas ilhas da ressonância $(2,1)$ presentes na Figura 16.

\begin{tabular}{|l|c|c|}
\hline & $\begin{array}{c}\text { Sistema sem controle } \\
\text { (Figura 16(a)) }\end{array}$ & $\begin{array}{c}\text { Sistema controlado } \\
\text { (Figura 16(b)) }\end{array}$ \\
\hline Energia Adimensional Mínima & 1,872 & 1,766 \\
Energia Adimensional Máxima & 2,398 & 2,505 \\
Ganho de Energia Adimensional & 0,527 & 0,739 \\
Momento Adimensional Mínimo & 1,531 & 1,380 \\
Momento Adimensional Máximo & 2,147 & 2,235 \\
Ganho de Momento Adimensional & 0,616 & 0,855 \\
Velocidade Adimensional Mínima & 0,837 & 0,810 \\
Velocidade Adimensional Máxima & 0,907 & 0,913 \\
Ganho de Velocidade Adimensional & 0,069 & 0,103 \\
\hline
\end{tabular}

\subsection{COMPROVAÇÃO EXPERIMENTAL EM UMA VÁLVULA DE ONDAS PROGRESSIVAS (TWT)}

A validade do método de controle do caos proposto na Ref. [33] e discutido neste capítulo foi comprovada na Ref. [34] através de experimentos realizados em uma válvula de ondas progressivas [35, 36], mais conhecida como TWT (do inglês, traveling wave tube). No TWT, um feixe de elétrons é confinado por um campo magnético axial e ele se propaga sob a ação de ondas eletrostáticas. O equipamento utilizado é longo o suficiente para permitir o surgimento de processos não lineares [34]. Além disso, as características do equipamento permitem o controle dos parâmetros do feixe de elétrons e das ondas 
eletrostáticas, o que torna o TWT um dispositivo ideal para o estudo da interação-partícula.

No experimento descrito na Ref. [34], um feixe de elétrons interage com duas ondas eletrostáticas de $30 \mathrm{MHz}$ e velocidades de fase diferentes. O termo de controle, nesse caso, é uma terceira onda eletrostática de baixa amplitude e frequência igual a $60 \mathrm{MHz}$. Os parâmetros da onda de controle foram obtidos teoricamente a partir do método proposto na Ref. [33]. Os resultados experimentais descritos na Ref. [34] concordaram com as simulações numéricas apresentadas nessa mesma referência e mostram um aumento da coerência cinética do feixe após a adição da onda de controle.

Os resultados experimentais descritos na Ref. [34] também são comparáveis aos resultados teóricos apresentados neste capítulo. O experimento foi realizado no TWT do Laboratório de Física das Interações Iônicas e Moleculares (Laboratório PIIM) da Universidade de Aix-Marseille na França. No período de março de 2014 a fevereiro de 2015, a autora realizou um estágio de pesquisa no Laboratório PIIM, sob a supervisão do Prof. Dr. Fabrice Doveil, pesquisador responsável pelo TWT e pelo experimento apresentado na Ref. [34]. O TWT do Laboratório PIIM passou por algumas modificações para aumentar o desempenho do equipamento e permitir a realização de novos experimentos. Durante o estágio, a autora realizou um trabalho experimental e teve a oportunidade de unir conhecimentos teóricos e experimentais. Ela participou diretamente da montagem e da caracterização do novo TWT, realizou cálculos analíticos, simulações numéricas e coleta de dados experimentais para análise, como descrito na Ref. [72]. O trabalho experimental realizado durante o estágio foi apresentado em conferências e publicado nos anais dos eventos, como pode ser visto nas Refs. [52, 53].

\subsubsection{Montagem experimental e caracterização de uma válvula de ondas progressivas (TWT)}

Válvulas de ondas progressivas ou TWTs (do inglês, traveling wave tubes) [35, 36] são equipamentos idealmente concebidos para o estudo da interação onda-partícula, pois eles permitem que diversos parâmetros do sistema sejam controlados. Em um TWT, utiliza-se um gerador de formas de ondas arbitrárias que permite o controle do número de modos, da frequência, da amplitude e da fase de cada modo individual. No TWT, o feixe de elétrons também é produzido com controle da energia, da intensidade e da temperatura. Além disso, ao 
final do equipamento é utilizado um detector de energia [73] que fornece a distribuição de energia axial média do feixe com uma resolução menor do que $0,5 \mathrm{eV}$.

Os principais componentes de um TWT são um canhão de elétrons, um detector de velocidade dos elétrons, uma espira magnética, e uma estrutura de ondas lentas formada por uma hélice, onde as ondas eletrostáticas se propagam, com quatro antenas que podem se mover axialmente ao longo da válvula [34, 36]. A Figura 19 mostra uma representação esquemática de um TWT.

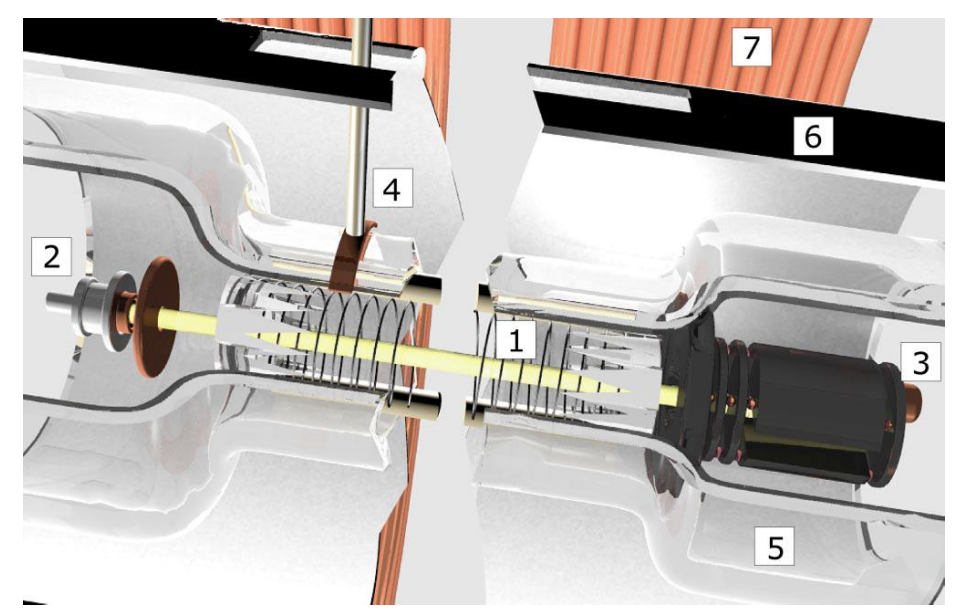

Figura 19: Estrutura de uma válvula de ondas progressivas (TWT): (1) hélice; (2) canhão de elétrons; (3) detector de velocidade dos elétrons; (4) antena móvel; (5) tubo de vácuo; (6) cilindro para aterramento de RF; (7) espira magnética.

Fonte: Referência [34].

Recentemente, o TWT do Laboratório de Física das Interações Iônicas e Moleculares (Laboratório PIIM) passou por uma série de modificações e sua hélice foi completamente substituída por um novo modelo. Após a montagem do equipamento, é necessário caracterizá-lo, o que inclui a determinação da relação de dispersão para a nova estrutura de ondas lentas, a amplitude dos campos elétrico e magnético através dessa estrutura, as velocidades de fase e de grupo das ondas, entre outras coisas. O Apêndice D mostra os principais resultados referentes à caracterização do novo TWT do Laboratório PIIM [72]. Esses resultados experimentais foram obtidos pela autora durante o estágio que ela realizou no laboratório. 


\subsection{CONCLUSÃO}

Nesta seção, foi apresentado um método de controle do caos desenvolvido na Ref. [33] para sistemas Hamiltonianos quase integráveis. A validade do método foi comprovada experimentalmente na Ref. [34] para um feixe de elétrons se movendo sob a ação de ondas eletrostáticas, um sistema semelhante ao analisado nesta tese.

O método de controle foi aplicado de forma teórica ao sistema em estudo e verificou-se que a amplitude do termo de controle é muito menor do que a amplitude da perturbação originalmente aplicada ao sistema. Um termo de controle com baixa amplitude não altera a estrutura de ilhas primárias no espaço de fases, mas torna o sistema controlado muito mais regular.

A partir dos resultados obtidos, foi possível apresentar uma análise qualitativa sobre a ação do termo de controle e como ele contribui para as características observadas no sistema controlado. Além de regularizar o sistema, o controle do caos também pode ser utilizado para melhorar o processo de aceleração regular de partículas. No sistema controlado, verificou-se que uma partícula com energia inicial menor do que no sistema original pode ser mais acelerada e atingir uma energia final maior do que ela atingiria no sistema sem o controle do caos.

Os resultados teóricos apresentados neste capítulo sobre controle de caos concordam com os resultados experimentais descritos na Ref. [34]. A experiência foi realizada em uma válvula de ondas progressivas [35, 36], mais conhecida como TWT (do inglês, traveling wave tube), do Laboratório de Física das Interações Iônicas e Moleculares (Laboratório PIIM) da Universidade de Aix-Marseille, França. Durante o desenvolvimento desta tese, a autora realizou um estágio de pesquisa de um ano no TWT do Laboratório PIIM para conhecer o equipamento e verificar como ele é utilizado em investigações experimentais sobre a interação onda-partícula. 


\section{CONCLUSÕES E PERSPECTIVAS}

Nesta tese, foi analisada a interação entre uma partícula relativística se movendo em um campo magnético uniforme e uma onda eletrostática e estacionária dada na forma de pulsos periódicos infinitesimais. A partir da Hamiltoniana do sistema, foi obtido um mapa exato e completamente explícito que descreve a evolução temporal da partícula $[16,17]$. Utilizando o mapa do sistema também foi possível construir o espaço de fases e analisar quando o comportamento do sistema é regular ou caótico e como ocorre a transição para o caos à medida que os parâmetros da onda aumentam.

O mapa obtido pode ser considerado como uma versão relativística e magnetizada do mapa padrão clássico, embora a posição das ressonâncias seja diferente nos dois mapas [16]. A partir da Hamiltoniana e do mapa que descrevem o sistema em estudo, obtém-se a posição aproximada das ressonâncias primárias no espaço de fases [50]. Quando a amplitude da onda eletrostática é pequena, as fórmulas obtidas fornecem a posição exata dos pontos periódicos de cada ressonância. Entretanto, o mapa do sistema apresenta uma forte não linearidade com relação à amplitude da onda e, portanto, o aumento desse parâmetro provoca o deslocamento dos pontos periódicos e das ilhas de ressonância. Isso não acontece com o mapa padrão clássico, para o qual os pontos periódicos estão localizados na mesma posição para qualquer valor da amplitude da onda.

Expandindo a Hamiltoniana do sistema em primeira ordem, mostrou-se que ela é perturbada por um número infinito de termos com o mesmo número de rotação $[50,51]$. Todos esses termos atuam na mesma superfície racional e podem gerar ilhas na mesma região do espaço de fases. A superposição de termos ressonantes com o mesmo número de rotação altera o número de cadeias de ilhas em função dos parâmetros da onda. Quando o período ou o número de onda são próximos de seus valores mínimos, o número de cadeias no espaço de fases também é mínimo. Aumentando o período ou o número de onda, o número de cadeias de ilhas também aumenta, até que para valores suficientemente elevados desses parâmetros, todas as ressonâncias apresentam mais de uma cadeia no espaço de fases, o que não é comumente observado na literatura [14, 54].

Utilizando o mapa do sistema, construiu-se o espaço de parâmetros que representa o número de cadeias de ilhas em função do período e do número de onda. Verificou-se que para todas as ressonâncias, as fronteiras que separam duas regiões com um número diferente de cadeias respeitam a mesma lei de potência [50]. Essa lei de potência está 
relacionada à amplitude dos vários termos ressonantes que possuem o mesmo número de rotação. Além dos espaços de parâmetros obtidos numericamente para todas as ressonâncias, também foi possível obter analiticamente o intervalo de parâmetros da onda eletrostática que correspondem a um dado número de cadeias de ilhas para as principais ressonâncias do sistema [50]. É importante notar que, em todos os casos, a expansão em primeira ordem determina corretamente o número de cadeias de ilhas mesmo para valores de parâmetros para os quais a aproximação para a posição dos pontos periódicos perde precisão.

Verificou-se também que os termos ressonantes que atuam no sistema são simétricos e, como consequência, todas as ressonâncias primárias possuem um número par de ilhas que estão agrupadas em cadeias no espaço de fases [50]. As cadeias por sua vez podem conter um número par ou ímpar de ilhas. Isso significa que as cadeias que possuem um número ímpar de ilhas sempre ocorrem aos pares no espaço de fases, pois o número total de ilhas da ressonância é sempre par. Portanto, conclui-se que de acordo com a simetria dos termos ressonantes, a dinâmica de um sistema twist quase integrável pode ser dominada por múltiplas cadeias isócronas, ao contrário do cenário usual de cadeias únicas.

Para o sistema em estudo, o número de cadeias de ilhas cresce sem limites com os parâmetros do sistema [50, 51]. Observa-se, entretanto, que esse resultado não é particular. $\mathrm{O}$ mesmo fenômeno pode ocorrer em sistemas twist quase integráveis que apresentam um número infinito de termos ressonantes atuando na mesma superfície racional. Por outro lado, quando o número de termos ressonantes com o mesmo número de rotação é finito, o número de cadeias também varia com os parâmetros, mas ele não aumenta sem limites. Nesse caso, existe um conjunto finito para os possíveis números de cadeias que é determinado pelos termos ressonantes que possuem o mesmo número de rotação e por sua superposição. Para sistemas twist quase integráveis que apresentam apenas um termo ressonante, o número de cadeias isócronas não varia com os parâmetros. O termo ressonante determina completamente o número de cadeias no espaço de fases e o número de ilhas em cada cadeia, como pode ser visto na Ref. [51].

A variação no número de cadeias em função dos parâmetros do sistema é um fenômeno importante, pois nas ilhas de ressonância a partícula recebe energia da onda e pode ser acelerada de forma regular [15-17]. Para acelerar a partícula nas ilhas de uma dada ressonância, é necessário conhecer a posição dos pontos periódicos e o número de cadeias. Dessa forma, as condições iniciais da partícula podem ser ajustadas para que ela atinja a aceleração máxima.

Nesta tese, considerou-se a ressonância principal do sistema, para a qual cada 
ciclo da onda eletrostática corresponde a um giro completo da partícula no plano perpendicular à direção do campo magnético. Quando o período da onda eletrostática é ligeiramente maior do que o período ciclotrônico, a ressonância principal está localizada no nível mais baixo de energia. Nessa ressonância, o aumento dos parâmetros da onda eletrostática provoca o deslocamento dos pontos hiperbólicos e as ilhas de ressonância passam a apresentar uma forma triangular que contribui para a aceleração de partículas [16]. Utilizando o mapa do sistema, foi possível calcular os parâmetros da onda para os quais os pontos hiperbólicos são deslocados até o nível mínimo de energia. Dessa forma, uma partícula com velocidade inicial próxima à sua velocidade de repouso pode ser acelerada e atingir velocidades bastante elevadas.

Entretanto, existe um limite para o processo de aceleração regular. Quando os pontos elípticos da ressonância principal perdem estabilidade, as ilhas de ressonância primária deixam de existir e não é mais possível acelerar a partícula de forma regular. Logo, para que a aceleração da partícula seja máxima, os pontos hiperbólicos da ressonância principal devem atingir o nível de energia mínima antes dos pontos elípticos perderem estabilidade. Essa condição fornece um intervalo de parâmetros da onda eletrostática para os quais a partícula pode ser acelerada de forma regular nas ilhas da ressonância principal [16].

Além disso, é importante destacar que a ressonância principal e o processo de aceleração regular só estão presentes no espaço de fases do sistema quando os efeitos relativísticos de correção de massa são considerados [16]. Se os efeitos relativísticos forem erroneamente ignorados, as ilhas de ressonância são substituídas por toros invariantes [70].

Nesta tese, também foi apresentado um estudo sobre controle de caos em sistemas descritos por Hamiltonianas quase integráveis. Para tanto, o método de controle desenvolvido na Ref. [33] foi aplicado ao sistema em estudo. A partir desse método, calculouse um termo de controle que deve ser adicionado à Hamiltoniana do sistema. O termo de controle obtido foi simplesmente uma segunda onda eletrostática e estacionária com amplitude muito menor do que a amplitude da perturbação originalmente aplicada ao sistema [17]. Um termo de controle tão pequeno não altera a estrutura de ilhas primárias no espaço de fases, embora a dinâmica controlada seja muito mais regular. Enquanto no sistema original as trajetórias caóticas preenchem uma grande área do espaço de fases, no sistema controlado as trajetórias caóticas são confinadas em pequenas regiões e quase todas as órbitas são regulares.

Além de regularizar o sistema, verificou-se que o controle do caos pode ser utilizado para melhorar o processo de aceleração regular de partículas [17]. Na dinâmica controlada, a redução de regiões caóticas no espaço de fases restaura órbitas periódicas e 
quase periódicas que são responsáveis por acelerar as partículas de forma regular. Logo, no sistema controlado, uma partícula com energia inicial menor do que no sistema original pode ser mais acelerada e atingir uma energia final maior do que ela atingiria no sistema sem controle.

Também foi construído um espaço de fases considerando apenas a interação da partícula relativística com o campo magnético e o termo de controle. A partir dos resultados obtidos, foi possível analisar de forma qualitativa como o termo de controle atua no sistema e como ocorre o acoplamento das duas ondas eletrostáticas que dá origem às características observadas no sistema controlado.

O método de controle utilizado nesta tese é dito global, pois ele atua em todo o espaço de fases, criando toros invariantes e reduzindo as regiões de comportamento caótico. Nas próximas etapas deste trabalho, serão analisados novos métodos de controle do caos, incluindo métodos que atuam localmente em uma região pré-determinada do espaço de fases, assim como a criação de um único toro robusto no espaço de fases. $\mathrm{O}$ objetivo é controlar o caos no sistema com um custo energético menor do que o observado na Ref. [17] e no Capítulo 5 e melhorar o processo de aceleração regular de partículas a partir de baixas energias iniciais como descrito no Capítulo 4 e na Ref. [16].

No Capítulo 3 e nas Refs. [50, 51], foram apresentados os resultados obtidos sobre as ressonâncias primárias do sistema. Nas próximas etapas, será feito um estudo mais detalhado sobre as bifurcações sofridas pelos pontos periódicos e sobre os mecanismos que levam ao surgimento de novas cadeias de ilhas à medida que os parâmetros da onda aumentam. O estudo das ressonâncias primárias trará uma melhor compreensão sobre a dinâmica do sistema, a transição para o caos global e a possibilidade de controle do caos no espaço de fases.

Além disso, será desenvolvido um estudo sobre o número de cadeias e como esse número varia de acordo com os parâmetros para sistemas twist quase integráveis que são perturbados por um número finito de termos ressonantes com o mesmo número de rotação. Através desse estudo, será possível generalizar os resultados obtidos até o momento e desenvolver uma teoria capaz de identificar o número de cadeias de ilhas isócronas presentes nas seções de Poincaré de sistemas twist quase integráveis, um problema que permanece em aberto no Teorema do Ponto Fixo de Poincaré-Birkhoff [14, 40, 58, 65].

Como mencionado no Capítulo 5, válvulas de ondas progressivas [35, 36], ou TWTs (do inglês, traveling wave tubes), são equipamentos idealmente concebidos para a análise da interação onda-partícula. Durante o doutorado, a autora realizou um estágio de um 
ano no TWT do Laboratório de Física das Interações Iônicas e Moleculares (Laboratório PIIM) da Universidade de Aix-Marseille, França. O estágio foi realizado sob a supervisão do Prof. Dr. Fabrice Doveil. Em seu doutorado na Universidade de São Paulo, a autora investigou a interação onda-partícula de forma teórica. No Laboratório PIIM, ela teve a oportunidade de estudar experimentalmente esse tipo de interação através de um TWT. Durante o estágio, ela participou diretamente da montagem e da caracterização do novo TWT do laboratório. Ela realizou cálculos analíticos, simulações numéricas e coleta de dados experimentais para análise, como descrito em detalhes na Ref. [72]. Através do estágio, foi possível unir conhecimentos teóricos e experimentais, o que contribuiu de forma significativa para o desenvolvimento desta tese. O trabalho realizado durante o estágio foi apresentado em conferências e publicado nos anais dos eventos, como pode ser visto nas Refs. [52, 53].

A realização do estágio no Laboratório PIIM trouxe a possibilidade de futuras colaborações nas áreas de interação onda-partícula e controle do caos. Após a conclusão das modificações realizadas no TWT do Laboratório PIIM, o dispositivo será utilizado na investigação do comportamento de feixes de baixa densidade [74-79] e na possibilidade de controle de sua dinâmica $[17,34,80]$. No TWT, também serão realizados experimentos com feixes de alta densidade. O objetivo é analisar a influência da dinâmica caótica dos elétrons nos efeitos autoconsistentes [81] responsáveis pelo crescimento da onda [82] quando a intensidade do feixe aumenta.

Além disso, os experimentos realizados no TWT serão utilizados para verificar a validade de códigos numéricos [83], analisar o comportamento de pacotes de elétrons emitidos com uma fase controlada em relação às ondas [84], e investigar a interação entre partículas rápidas (essencialmente partículas alfa) ${ }^{9}$ e ondas de Alfvén em um reator de fusão [5], o que é um problema fundamental para o ITER (International Thermonuclear Experimental Reactor).

Os experimentos mencionados acima serão realizados no TWT do Laboratório PIIM da Universidade de Aix-Marseille, e a análise teórica será feita em conjunto pelo Laboratório PIIM e pelo Grupo de Controle de Oscilações da Universidade de São Paulo.

\footnotetext{
${ }^{9}$ Em um reator de fusão, a relação $E / m$ entre a energia e a massa de partículas rápidas é comparável à relação $E / m$ para elétrons. Por esse motivo, os resultados obtidos com elétrons no TWT podem ser utilizados em estimativas para partículas rápidas em reatores de fusão.
} 


\section{REFERÊNCIAS BIBLIOGRÁFICAS}

[1] R. C. Davidson and H. Qin, Physics of intense charged particle beams in high energy accelerators (World Scientific, London, 2001).

[2] D. A. Edwards and M. J. Syphers, An introduction to the physics of high energy accelerators (Wiley-VCH, Weinheim, 2004).

[3] P. K. Shukla, N. N. Rao, M. Y. Yu and N. L. Tsintsadze, Relativistic nonlinear effects in plasmas, Phys. Rep. 138, 1 (1986).

[4] N. J. Fisch, Theory of current drive in plasmas, Rev. Mod. Phys. 59, 175 (1987).

[5] H. L. Berk, B. N. Breizman and Huanchun Ye, Scenarios for the nonlinear evolution of alpha-particle-induced Alfvén wave instability, Phys. Rev. Lett. 68, 3563 (1992).

[6] C. F. F. Karney, Stochastic ion heating by a lower hybrid wave, Phys. Fluids 21, 1584 (1978).

[7] G. Corso and F. B. Rizzato, Stochastic cyclotron dynamics in the interaction of waves and low-energy particles, J. Plasma Phys. 49, 425 (1993).

[8] C. B. Wang, C. S. Wu and P. H. Yoon, Heating of ions by Alfvén waves via nonresonant interactions, Phys. Rev. Lett. 96, 125001 (2006).

[9] T. Tajima and J. M. Dawson, Laser electron accelerator, Phys. Rev. Lett. 43, 267 (1979).

[10] R. Spektor and E. Y. Choueiri, Ion acceleration by beating electrostatic waves: Domain of allowed acceleration, Phys. Rev. E 69, 046402 (2004).

[11] E. Malamud (Ed.), Accelerators and beams: Tools of discovery and innovation (3rd ed., Division of Physics of Beams of the American Physical Society, 2010).

[12] Accelerators for America's future (U.S. Department of Energy's Office of Science, 2010).

[13] J. T. Mendonça, Theory of photon acceleration (IOP Publishing, Bristol, UK, 2001).

[14] A. J. Lichtenberg and M. A. Lieberman, Regular and chaotic dynamics (2nd ed., Springer, New York, 1992).

[15] R. Pakter and G. Corso, Improving regular acceleration in the nonlinear interaction of particles and waves, Phys. Plasmas 2, 4312 (1995).

[16] M. C. de Sousa, F. M. Steffens, R. Pakter and F. B. Rizzato, Standard map in magnetized relativistic systems: Fixed points and regular acceleration, Phys. Rev. E 82, 026402 (2010). 
[17] M. C. de Sousa, I. L. Caldas, F. B. Rizzato, R. Pakter and F. M. Steffens, Controlling chaos in wave-particle interactions, Phys. Rev. E 86, 016217 (2012).

[18] J. R. Cary, Vacuum magnetic fields with dense flux surfaces, Phys. Rev. Lett. 49, 276 (1982).

[19] J. R. Cary and J. D. Hanson, Stochasticity reduction, Phys. Fluids 29, 2464 (1986).

[20] T. Shinbrot, C. Grebogi, E. Ott and J. A. Yorke, Using small perturbations to control chaos, Nature 363, 411 (1993).

[21] K. H. Spatschek, M. Eberhard and H. Friedel, On models for magnetic field line diffusion, Phys. Mag. 2, 85 (1998).

[22] M. S. Baptista and I. L. Caldas, Easy-to-implement method to target nonlinear systems, Chaos, 8, 290 (1998).

[23] D. J. Gauthier, Controlling chaos, Am. J. Phys. 71, 750 (2003).

[24] S. R. Hudson, Destruction of invariant surfaces and magnetic coordinates for perturbed magnetic fields, Phys. Plasmas 11, 677 (2004).

[25] C. Chandre, M. Vittot, G. Ciraolo, Ph. Ghendrih and R. Lima, Control of stochasticity in magnetic field lines, Nucl. Fusion 46, 33 (2006).

[26] S. L. T. de Souza, I. L. Caldas, R. L. Viana, Damping control law for a chaotic impact oscillator, Chaos, Solitons and Fractals 32, 745 (2007).

[27] M. A. F. Sanjuán and C. Grebogi, Recent progress in controlling chaos (World Scientific, London, 2010).

[28] L. S. Pontryagin, V. G. Boltyanskii, R. V. Gamkrelidze and E. F. Mishchenko, The mathematical theory of optimal processes (Wiley, New York, 1961).

[29] E. Ott, C. Grebogi and J. A. Yorke, Controlling chaos, Phys. Rev. Lett. 64, 1196 (1990).

[30] R. Lima and M. Pettini, Suppression of chaos by resonant parametric perturbations, Phys. Rev. A 41, 726 (1990).

[31] Y. C. Lai, M. Ding and C. Grebogi, Controlling Hamiltonian chaos, Phys. Rev. E 47, 86 (1993).

[32] E. Ott and M. Spano, Controlling chaos, Phys. Today 48(5), 34 (1995).

[33] M. Vittot, Perturbation theory and control in classical or quantum mechanics by an inversion formula, J. Phys. A 37, 6337 (2004).

[34] C. Chandre, G. Ciraolo, F. Doveil, R. Lima, A. Macor, and M. Vittot, Channeling chaos by building barriers, Phys. Rev. Lett. 94, 074101 (2005). 
[35] J. R. Pierce, Traveling Wave Tubes (Van Nostrand, New York, 1950).

[36] A. S. Gilmour Jr., Principles of Traveling Wave Tubes (Artech House, London, 1994).

[37] G. Ciraolo, F. Briolle, C. Chandre, E. Floriani, R. Lima, M. Vittot, M. Pettini, C. Figarella, and P. Ghendrih, Control of Hamiltonian chaos as a possible tool to control anomalous transport in fusion plasmas, Phys. Rev. E 69, 056213 (2004).

[38] G. Ciraolo, C. Chandre, R. Lima, M. Vittot, M. Pettini, C. Figarella, and P. Ghendrih, Controlling chaotic transport in a Hamiltonian model of interest to magnetized plasmas, J. Phys. A 37, 3589 (2004).

[39] G. Ciraolo, C. Chandre, R. Lima, M. Vittot, M. Pettini, and P. Ghendrih, Tailoring phase space: A way to control Hamiltonian transport, Europhys. Lett. 69, 879 (2005).

[40] L. E. Reichl, The transition to chaos: Conservative classical systems and quantum manifestations (2nd ed., Springer, New York, 2004).

[41] J. D. Szezech, Jr., I. L. Caldas, S. R. Lopes, R. L. Viana, and P. J. Morrison, Transport properties in nontwist area-preserving maps, Chaos 19, 043108 (2009).

[42] G. Corso and F. B. Rizzato, Manifold reconnection in chaotic regimes, Phys. Rev. E 58, 8013 (1998).

[43] S. D. Prado and G. Corso, Manifold reconnection and diffusion in strong chaos, Physica D 142, 217 (2000).

[44] T. M. Corrêa da Silva, R. Pakter, F. B. Rizzato, M. C. de Sousa, I. L. Caldas, and F. M. Steffens, Chaotic particle heating due to an obliquely propagating wave in a magnetized plasma, Phys. Rev. E 88, 013101 (2013).

[45] N. A. Krall and A. W. Trivelpiece, Principles of plasma physics (McGraw-Hill, New York, 1973).

[46] S. M. Lund and R. C. Davidson, Warm-fluid description of intense beam equilibrium and electrostatic stability properties, Phys. Plasmas 5, 3028 (1998).

[47] R. Pakter, G. Corso, T. S. Caetano, D. Dillenburg, and F. B. Rizzato, Bifurcations leading to stochasticity in a cyclotron-maser system, Phys. Plasmas 1, 4099 (1994).

[48] F. B. Rizzato and R. Pakter, Gap bifurcations in nonlinear dynamical systems, Phys. Rev. Lett. 89, 184102 (2002).

[49] M. C. de Sousa, Dinâmica Hamiltoniana e caos (Trabalho de Graduação Interdisciplinar, Graduação em Física, Universidade Presbiteriana Mackenzie, São Paulo, 2009).

[50] M. C. de Sousa, I. L. Caldas, A. M. Ozorio de Almeida, F. B. Rizzato and R. Pakter, Alternate islands of multiple isochronous chains in wave-particle interactions, Phys. Rev. E 88, 064901 (2013). 
[51] M. C. de Sousa, I. L. Caldas, A. M. Ozorio de Almeida, F. B. Rizzato and R. Pakter, Multiple island chains in wave-particle interactions, J. Phys.: Conf. Ser., aceito para publicação no volume dedicado ao XVII Colóquio Brasileiro de Dinâmica Orbital CBDO 2014 (2015).

[52] M. Caetano de Sousa, D. Guyomarc'h and F. Doveil, A TWT-upgrade to study waveparticle interactions, Proceedings of the $42^{\text {nd }}$ European Physical Society Conference on Plasma Physics, European Physical Society, Lisboa, Portugal, Europhysics Conference Abstracts 39E, P5.406 (2015).

[53] F. Doveil, M. C. de Sousa, D. Guyomarc'h, A. Kahli and Y. Elskens, A TWT upgrade to study wave-particle interactions in plasma, Proceedings of the $57^{\text {th }}$ Annual Meeting of the APS Division of Plasma Physics, American Physical Society, Savannah, Estados Unidos, a ser publicado (2015).

[54] L. H. A. Monteiro, Sistemas dinâmicos (2. ed., Livraria da Física, São Paulo, 2006).

[55] J. R. Reitz and F. J. Milford, Foundations of electromagnetic theory (Addison-Wesley, Reading, 1960).

[56] J. D. Jackson, Classical electrodynamics (3rd ed., Wiley, New York, 1999).

[57] K. Zhao, Q. Zhang, M. Chini, Y. Wu, X. Wang and Z. Chang, Tailoring a 67 attosecond pulse through advantageous phase-mismatch, Optics Letters 37, 3891 (2012).

[58] M. V. Berry, Regular and irregular motion, AIP Conf. Proc. 46, 16 (1978).

[59] A. M. Ozorio de Almeida, Hamiltonian systems: Chaos and quantization (Cambridge University Press, Cambridge, 1988).

[60] J. P. Van Der Weele, T. P. Valkering, H. W. Capel and T. Post, The birth of twin Poincaré-Birkhoff chains near 1:3 resonance, Physica A 153, 283 (1988).

[61] G. A. Oda and I. L. Caldas, Dimerized island chains in tokamaks, Chaos, Solitons and Fractals 5, 15 (1995).

[62] D. del-Castillo-Negrete, J. M. Greene and P. J. Morrison, Area preserving nontwist maps: periodic orbits and transition to chaos, Physica D 91, 1 (1996).

[63] E. Petrisor, J. H. Misguich and D. Constantinescu, Reconnection in a global model of Poincaré map describing dynamics of magnetic field lines in a reversed shear tokamak, Chaos, Solitons and Fractals 18, 1085 (2003).

[64] G. Voyatzis, E. Meletlidou and S. Ichtiaroglou, Large-scale chaos for arbitrarily small perturbations in non-twist Hamiltonian systems, Chaos, Solitons and Fractals 14, 1179 (2002).

[65] G. D. Birkhoff, On the periodic motions of dynamical systems, Acta Math. 50, 359 (1927). 
[66] R. Egydio de Carvalho and A. M. Ozorio de Almeida, Integrable approximation to the overlap of resonances, Phys. Lett. A 162, 457 (1992).

[67] J. S. E. Portela, I. L. Caldas, and R. L. Viana, Tokamak magnetic field lines described by simple maps, Eur. Phys. J. Special Topics 165, 195 (2008).

[68] G. H. Walker and J. Ford, Amplitude instability and ergodic behavior for conservative nonlinear oscillator systems, Phys. Rev. 188, 416 (1969).

[69] J. P. Van Der Weele, H. W. Capel, T. P. Valkering and T. Post, The squeeze effect in non-integrable Hamiltonian systems, Physica A 147, 499 (1988).

[70] J.-H. Kim and H.-W. Lee, Chaos in the relativistic cyclotron motion of a charged particle, Phys. Rev. E 54, 3461 (1996).

[71] M. Vittot, C. Chandre, G. Ciraolo and R. Lima, Localized control for non-resonant Hamiltonian systems, Nonlinearity 18, 423 (2005).

[72] M. C. de Sousa, Controle de caos na interação onda-partícula (Controlling chaos in wave-particle interactions) (Relatório Científico, Estágio de Pesquisa no Exterior Nível Doutorado Direto, Processo FAPESP número 2013/01335-6, Aix-Marseille Université, Marselha, 2015).

[73] D. Guyomarc'h and F. Doveil, A trochoidal analyzer to measure the electron beam energy distribution in a traveling wave tube, Rev. Sci. Instrum. 71, 4087 (2000).

[74] F. Doveil, D. F. Escande and A. Macor, Experimental observation of nonlinear synchronization due to a single wave, Phys. Rev. Lett. 94, 085003 (2005).

[75] A. Macor, F. Doveil and Y. Elskens, Electron climbing a "devil's staircase" in waveparticle interaction, Phys. Rev. Lett. 95, 264102 (2005).

[76] F. Doveil, Kh. Auhmani, A. Macor and D. Guyomarc'h, Experimental observation of resonance overlap responsible for Hamiltonian chaos, Phys. Plasmas 12, 010702 (2005).

[77] M. Buchanan, Thesis: Richness in simplicity, Nature Physics 2, 429 (2006).

[78] F. Doveil, A. Macor and Y. Elskens, Direct observation of a "devil's staircase" in wave-particle interaction, Chaos 16, 033103 (2006).

[79] A. Ruzzon, Y. Elskens and F. Doveil, A symplectic, symmetric algorithm for spatial evolution of particles in a time-dependent field, Comm. Nonlinear Sci. Numer. Simulat., 17, 769 (2012).

[80] A. Macor, F. Doveil, C. Chandre, G. Ciraolo, R. Lima and M. Vittot, Channeling chaotic transport in a wave-particle experiment, Eur. Phys. J. D 41, 519-530 (2007); and erratum 44, 201 (2007).

[81] F. Doveil and A. Macor, Two regimes of self-consistent heating of charged particles, 
Phys. Rev. E 84, 045401 (2011).

[82] S. I. Tsunoda, F. Doveil and J. H. Malmberg, Experimental test of the quasilinear theory of the interaction between a weak warm electron beam and a spectrum of waves, Phys. Rev. Lett. 58, 1112 (1987).

[83] P. Bernardi, F. André, J-F. David, A. Leclair and F. Doveil, Efficient time-domain simulations of a helix traveling-wave tube, IEEE Trans. El. Dev. 58, 1761 (2011).

[84] A. Macor, F. Doveil and E. Garabedian, Electron packets to investigate nonlinear phenomena in wave-particle interaction, Nonlinear Phenomena in Complex Systems 10, 180 (2007).

[85] D. Guyomarc'h, Un tube à onde progressive pour l'étude de la turbulence plasma (Tese de Doutorado, Doutorado em Física, Université de Provence, Marselha, 1996). 


\section{APÊNDICE A - Propriedades de simetria da onda eletrostática e estacionária dada na} forma de pulsos periódicos

Considere a onda eletrostática e estacionária dada pela Hamiltoniana expandida

$$
\varepsilon V=\frac{\varepsilon}{2 T} \sum_{l_{1}=-\infty}^{+\infty} \sum_{l_{2}=-\infty}^{+\infty} J_{l_{2}}(k \sqrt{2 I}) \cos \left(l_{2} \theta-\frac{2 \pi l_{1} t}{T}\right)
$$

Como visto na Seção 3.2, apenas os termos em (A1) para os quais $l_{1}$ e $l_{2}$ possuem o mesmo sinal (com $l_{1} \neq 0$ e $l_{2} \neq 0$ ) são ressonantes e podem gerar ilhas no espaço de fases. Portanto, a parte ressonante de (A1) é definida como

$$
\begin{aligned}
& \varepsilon V_{\text {res }}=\frac{\varepsilon}{2 T}\left[\sum_{l_{1}=-\infty}^{-1} \sum_{l_{2}=-\infty}^{-1} J_{l_{2}}(k \sqrt{2 I}) \cos \left(l_{2} \theta-\frac{2 \pi l_{1} t}{T}\right)+\sum_{l_{1}=1}^{+\infty} \sum_{l_{2}=1}^{+\infty} J_{l_{2}}(k \sqrt{2 I}) \cos \left(l_{2} \theta-\frac{2 \pi l_{1} t}{T}\right)\right] \\
& \varepsilon V_{\text {res }}=\frac{\varepsilon}{2 T}\left[\sum_{l_{1}=1}^{+\infty} \sum_{l_{2}=1}^{+\infty}(-1)^{l_{2}} J_{l_{2}}(k \sqrt{2 I}) \cos \left(-l_{2} \theta+\frac{2 \pi l_{1} t}{T}\right)+\sum_{l_{1}=1}^{+\infty} \sum_{l_{2}=1}^{+\infty} J_{l_{2}}(k \sqrt{2 I}) \cos \left(l_{2} \theta-\frac{2 \pi l_{1} t}{T}\right)\right], \\
& \varepsilon V_{\text {res }}=\frac{\varepsilon}{T} \sum_{l_{2}=2}^{+\infty} \sum_{l_{2}=1}^{+\infty} J_{l_{2}}(k \sqrt{2 I}) \cos \left(l_{2} \theta-\frac{2 \pi l_{1} t}{T}\right) .
\end{aligned}
$$

Devido à simetria da parte ressonante da onda, todos os termos para os quais $r$ é ímpar são cancelados aos pares. Apenas os termos com $r$ par atuam no sistema e eles geram um número par de ilhas para todas as ressonâncias primárias $(r, s)$. Logo, quando o número de ilhas em cada cadeia é ímpar, o número de cadeias deve ser par. Por outro lado, quando a ressonância apresenta um número par de ilhas em cada uma de suas cadeias, o número de cadeias pode ser tanto par quanto ímpar. 


\section{APÊNDICE B - Pontos periódicos presentes em todas as ressonâncias primárias}

A condição (22) para pontos periódicos determina os valores da variável $\theta$ nas ressonâncias primárias $(r, s)$. Entretanto, é possível demonstrar que alguns valores de $\theta$ são pontos periódicos para quaisquer valores de $r$ e $s$, ou seja, eles são pontos periódicos de todas as ressonâncias primárias do sistema.

Utilizando relações de soma e subtração entre funções trigonométricas, a expressão (22) pode ser reescrita como

$$
\begin{aligned}
G_{r, s}(\theta)= & \sum_{j=0}^{r-1}\left\{\left[\cos \theta \cos \left(\frac{2 \pi s}{r} j\right)-\operatorname{sen} \theta \operatorname{sen}\left(\frac{2 \pi s}{r} j\right)\right]\right. \\
& \times\left\{\operatorname{sen}\left[k \sqrt{2 I_{r, s}} \operatorname{sen} \theta \cos \left(\frac{2 \pi s}{r} j\right)\right] \cos \left[k \sqrt{2 I_{r, s}} \cos \theta \operatorname{sen}\left(\frac{2 \pi s}{r} j\right)\right]\right. \\
& \left.\left.+\operatorname{sen}\left[k \sqrt{2 I_{r, s}} \cos \theta \operatorname{sen}\left(\frac{2 \pi s}{r} j\right)\right] \cos \left[k \sqrt{2 I_{r, s}} \operatorname{sen} \theta \cos \left(\frac{2 \pi s}{r} j\right)\right]\right\}\right\}=0 .
\end{aligned}
$$

O segundo termo da equação (B1), para o qual $j=1$, é dado por

$$
\begin{aligned}
& {\left[\cos \theta \cos \left(\frac{2 \pi s}{r}\right)-\operatorname{sen} \theta \operatorname{sen}\left(\frac{2 \pi s}{r}\right)\right]} \\
& \times\left\{\operatorname{sen}\left[k \sqrt{2 I_{r, s}} \operatorname{sen} \theta \cos \left(\frac{2 \pi s}{r}\right)\right] \cos \left[k \sqrt{2 I_{r, s}} \cos \theta \operatorname{sen}\left(\frac{2 \pi s}{r}\right)\right]\right. \\
& \left.+\operatorname{sen}\left[k \sqrt{2 I_{r, s}} \cos \theta \operatorname{sen}\left(\frac{2 \pi s}{r}\right)\right] \cos \left[k \sqrt{2 I_{r, s}} \operatorname{sen} \theta \cos \left(\frac{2 \pi s}{r}\right)\right]\right\},
\end{aligned}
$$

enquanto o último termo, para o qual $j=r-1$, é dado por

$$
\begin{aligned}
{\left[\cos \theta \cos \left(\frac{2 \pi s}{r}\right)+\operatorname{sen} \theta \operatorname{sen}\left(\frac{2 \pi s}{r}\right)\right] } \\
\times\left\{\operatorname{sen}\left[k \sqrt{2 I_{r, s}} \operatorname{sen} \theta \cos \left(\frac{2 \pi s}{r}\right)\right] \cos \left[k \sqrt{2 I_{r, s}} \cos \theta \operatorname{sen}\left(\frac{2 \pi s}{r}\right)\right]\right. \\
\left.-\operatorname{sen}\left[k \sqrt{2 I_{r, s}} \cos \theta \operatorname{sen}\left(\frac{2 \pi s}{r}\right)\right] \cos \left[k \sqrt{2 I_{r, s}} \operatorname{sen} \theta \cos \left(\frac{2 \pi s}{r}\right)\right]\right\},
\end{aligned}
$$

onde foram utilizadas as relações 


$$
\cos \left(\frac{2 \pi s}{r}(r-1)\right)=\cos \left(\frac{2 \pi s}{r}\right)
$$

e

$$
\operatorname{sen}\left(\frac{2 \pi s}{r}(r-1)\right)=-\operatorname{sen}\left(\frac{2 \pi s}{r}\right)
$$

Somando as expressões (B2) e (B3) tem-se

$$
\begin{aligned}
& 2 \cos \theta \cos \left(\frac{2 \pi s}{r}\right) \operatorname{sen}\left[k \sqrt{2 I_{r, s}} \operatorname{sen} \theta \cos \left(\frac{2 \pi s}{r}\right)\right] \cos \left[k \sqrt{2 I_{r, s}} \cos \theta \operatorname{sen}\left(\frac{2 \pi s}{r}\right)\right] \\
& -2 \operatorname{sen} \theta \operatorname{sen}\left(\frac{2 \pi s}{r}\right) \operatorname{sen}\left[k \sqrt{2 I_{r, s}} \cos \theta \operatorname{sen}\left(\frac{2 \pi s}{r}\right)\right] \cos \left[k \sqrt{2 I_{r, s}} \operatorname{sen} \theta \cos \left(\frac{2 \pi s}{r}\right)\right] .
\end{aligned}
$$

De forma análoga, observa-se que todos os termos em (B1) se somam e se cancelam aos pares. Logo, a equação (B1) pode ser reescrita como

$$
\begin{aligned}
G_{r, s}(\theta)= & 2 \sum_{j=0}^{r / 2}\left\{\cos \theta \cos \left(\frac{2 \pi s j}{r}\right) \operatorname{sen}\left[k \sqrt{2 I_{r, s}} \operatorname{sen} \theta \cos \left(\frac{2 \pi s j}{r}\right)\right]\right. \\
& \times \cos \left[k \sqrt{2 I_{r, s}} \cos \theta \operatorname{sen}\left(\frac{2 \pi s j}{r}\right)\right]-\operatorname{sen} \theta \operatorname{sen}\left(\frac{2 \pi s j}{r}\right) \\
& \left.\times \operatorname{sen}\left[k \sqrt{2 I_{r, s}} \cos \theta \operatorname{sen}\left(\frac{2 \pi s j}{r}\right)\right] \cos \left[k \sqrt{2 I_{r, s}} \operatorname{sen} \theta \cos \left(\frac{2 \pi s j}{r}\right)\right]\right\}=0,
\end{aligned}
$$

para $r$ par, e

$$
\begin{aligned}
G_{r, s}(\theta)= & \cos \theta \operatorname{sen}\left(k \sqrt{2 I_{r, s}} \operatorname{sen} \theta\right)+2 \sum_{j=1}^{(r-1) / 2}\left\{\cos \theta \cos \left(\frac{2 \pi s j}{r}\right) \operatorname{sen}\left[k \sqrt{2 I_{r, s}} \operatorname{sen} \theta \cos \left(\frac{2 \pi s j}{r}\right)\right]\right. \\
& \times \cos \left[k \sqrt{2 I_{r, s}} \cos \theta \operatorname{sen}\left(\frac{2 \pi s j}{r}\right)\right]-\operatorname{sen} \theta \operatorname{sen}\left(\frac{2 \pi s j}{r}\right) \\
& \left.\times \operatorname{sen}\left[k \sqrt{2 I_{r, s}} \cos \theta \operatorname{sen}\left(\frac{2 \pi s j}{r}\right)\right] \cos \left[k \sqrt{2 I_{r, s}} \operatorname{sen} \theta \cos \left(\frac{2 \pi s j}{r}\right)\right]\right\}=0
\end{aligned}
$$

para $r$ ímpar.

A partir das expressões (B5) e (B6), observa-se que $G_{r, s}(\theta)=0$ para $\theta=0$; $\pi / 2 ; \pi ; 3 \pi / 2$, independente dos valores de $r$ e $s$. Logo, esses valores de $\theta$ são pontos periódicos para todas as ressonâncias primárias do sistema. 


\section{APÊNDICE C - Intervalo de parâmetros para um dado número de cadeias de ilhas nas} ressonâncias $(1, s) \mathbf{e}(2, s)$

Utilizando a expressão (B6), é possível determinar o intervalo de parâmetros para os quais as ressonâncias $(1, s)$ apresentam $M$ cadeias de ilhas. Substituindo $r=1$ na expressão (B6), tem-se

$$
G_{1, s}(\theta)=\cos \theta \operatorname{sen}\left(k \sqrt{2 I_{1, s}} \operatorname{sen} \theta\right)=0
$$

cujas soluções são

$$
\begin{aligned}
\cos \theta=0 & \Rightarrow \theta=\pi / 2 ; 3 \pi / 2, \\
\operatorname{sen} \theta=0 & \Rightarrow \theta=0 ; \pi,
\end{aligned}
$$

e

$$
k \sqrt{2 I_{1, s}} \operatorname{sen} \theta=n \pi \Rightarrow \theta=\operatorname{arcsen}\left(\frac{n \pi}{k \sqrt{2 I_{1, s}}}\right)
$$

onde $n$ é inteiro, $n \neq 0$ e

$$
-1<\frac{n \pi}{k \sqrt{2 I_{1, s}}}<1
$$

Em (C4), não são consideradas as soluções $\operatorname{sen} \theta=0 ; \pm 1$, pois elas já foram computadas nas expressões (C2) e (C3).

A condição (C5) impõe a existência de um valor mínimo e um valor máximo para $n$, com $\left|n_{\min }\right|=\left|n_{\max }\right|$. Por exemplo, se $n_{\max }=3$, (C4) apresenta $4 n_{\max }=12$ soluções que correspondem a $n= \pm 1, \pm 2, \pm 3$ (lembrando que cada valor de $n$ produz duas respostas diferentes para a função arcsen em (C4)).

De acordo com as expressões de (C2) a (C5), o número total de pontos periódicos nas ressonâncias $(1, s)$ é igual a $4\left(1+n_{\max }\right)$. Como foi visto na Seção 3.4, o número total de pontos periódicos também é dado por $2 M r$, onde $M$ é o número de cadeias de ilhas e para as ressonâncias $(1, s)$ tem-se $r=1$. A partir das condições $4\left(1+n_{\max }\right)=2 M \quad \mathrm{e}$ $\pi n_{\max } k^{-1}\left(2 I_{1, s}\right)^{-1 / 2}<1$, obtém-se o valor mínimo de $k$ para o qual as ressonâncias $(1, s)$ 
apresentam $M$ cadeias de ilhas no espaço de fases

$$
k>\frac{n_{\max } \pi}{\sqrt{2 I_{1, s}}} \Rightarrow k>\frac{(M-2) \pi}{2 \sqrt{2 I_{1, s}}}
$$

O valor máximo de $k$ que corresponde a $M$ cadeias de ilhas é igual ao valor mínimo de $k$ para $M+2$ cadeias de ilhas (como visto na Seção 3.4, o número de cadeias é sempre par para as ressonâncias $(1, s))$. Logo, é possível definir as fronteiras do intervalo de parâmetros para os quais as ressonâncias $(1, s)$ apresentam $M$ cadeias de ilhas no espaço de fases

$$
\frac{(M-2) \pi}{2 \sqrt{2 I_{1, s}}}<k<\frac{M \pi}{2 \sqrt{2 I_{1, s}}} .
$$

A partir da expressão (B5), demonstra-se de forma análoga que o intervalo de parâmetros para os quais as ressonâncias $(2, s)$ apresentam $M$ cadeias no espaço de fases é dado por

$$
\frac{(M-1) \pi}{\sqrt{2 I_{2, s}}}<k<\frac{M \pi}{\sqrt{2 I_{2, s}}} .
$$

As expressões (C7) e (C8) são válidas para todas as ressonâncias $(1, s)$ e $(2, s)$, independente do valor de $s$. Essas estimativas analíticas concordam bem com os resultados numéricos obtidos a partir do mapa (8). 


\section{APÊNDICE D - Relação de dispersão e velocidades de fase e de grupo para a nova válvula de ondas progressivas (TWT) do Laboratório de Física das Interações Iônicas e Moleculares (Laboratório PIIM)}

Em uma válvula de ondas progressivas [35, 36], mais conhecida como TWT (do inglês, traveling wave tube), um feixe de elétrons é confinado por um campo magnético axial e ele se propaga sob a ação de ondas eletrostáticas. TWTs são equipamentos ideais para o estudo da interação onda-partícula, pois eles permitem um grande controle dos parâmetros do feixe de elétrons e das ondas eletrostáticas. Um exemplo de aplicação do TWT é o experimento descrito na Ref. [34] que comprovou a eficácia do método de controle do caos desenvolvido na Ref. [33] e discutido no Capítulo 5 desta tese.

O experimento descrito na Ref. [34] foi realizado no TWT do Laboratório de Física das Interações Iônicas e Moleculares (Laboratório PIIM) da Universidade de AixMarseille, França. Recentemente, o TWT do Laboratório PIIM passou por uma série de modificações para aumentar o desempenho do equipamento, melhorar a precisão das medidas experimentais e permitir a realização de novos experimentos. Após a conclusão das modificações, o TWT será utilizado para investigar experimentalmente diversos aspectos da interação-onda partícula. A análise teórica será feita em conjunto pelo Laboratório PIIM e pelo Grupo de Controle de Oscilações da Universidade de São Paulo.

No período de março de 2014 a fevereiro de 2015, a autora realizou um estágio de pesquisa no TWT do Laboratório PIIM, sob a supervisão do Prof. Dr. Fabrice Doveil, pesquisador responsável pela experiência de controle do caos apresentada na Ref. [34]. Durante o estágio, a autora realizou um trabalho experimental, diferente do trabalho teórico realizado na Universidade de São Paulo. No Laboratório PIIM, ela participou diretamente da montagem e da caracterização do novo TWT, o que incluiu cálculos teóricos, simulações numéricas, coleta e análise de dados experimentais. O trabalho de montagem e caracterização do novo TWT foi apresentado em conferências e publicado nos anais dos eventos, como pode ser visto nas Ref. $[52,53]$. Os principais resultados referentes à caracterização do TWT são a relação de dispersão teórica e a comparação com os dados experimentais, a velocidade de fase e a velocidade de grupo teóricas. Tanto o cálculo das informações teóricas quanto a coleta de dados experimentais para análise foram realizados pela autora durante seu estágio no Laboratório PIIM, como descrito a seguir e na Ref. [72].

Os campos elétricos e magnéticos que se propagam através da estrutura de 
ondas lentas do TWT podem ser calculados a partir das equações de Maxwell [72, 85]. A relação de dispersão fria, isto é, sem a presença do feixe de elétrons, é obtida a partir das equações do campo eletromagnético, mais especificamente a partir da equação que determina a continuidade do campo magnético no interior do fio da hélice. No caso em que a velocidade de fase da onda é muito menor do que a velocidade da luz, a relação de dispersão teórica é dada aproximadamente por [72]

$$
2 \pi f=\omega=c k \operatorname{tg} \psi\left(\frac{\frac{U_{1}}{U_{2}}-\frac{I_{0}(a k)}{I_{1}(a k)}}{\varepsilon_{2} \frac{U_{4}}{U_{3}}-\frac{I_{1}(a k)}{I_{0}(a k)}}\right)^{1 / 2}=c k \operatorname{tg} \psi \sqrt{P_{1}},
$$

onde $k, \omega$ e $f$ são o número de onda, a frequência angular e a frequência em $\mathrm{Hz}$ da onda que se propaga através da hélice do TWT; $c$ é a velocidade da luz no vácuo; $\varepsilon_{2}$ é a constante dielétrica da fita isolante em poliimida que envolve a hélice, mantendo-a rígida; $\operatorname{tg} \psi=$ $p / 2 \pi a$, com $p$ o passo e $a$ o raio da hélice; $I_{j}$ são funções de Bessel modificadas; e $U_{j}$ são coeficientes obtidos a partir das condições de contorno do campo eletromagnético, como descrito em detalhes na Ref. [72].

A Figura 20 mostra a relação de dispersão teórica (curva azul) dada pela expressão (D1) e alguns pontos experimentais (em vermelho) obtidos pela autora durante seu estágio no Laboratório PIIM. A curva teórica concorda muito bem com os dados experimentais. Para todos os pontos experimentais, a diferença em relação à curva teórica é menor do que 1\% [72]. Na Figura 20, também são exibidos os pontos experimentais obtidos para a versão anterior do TWT (em verde). Comparando as barras de erro para os dois conjuntos de pontos experimentais, observa-se que a nova hélice e o novo sistema de medida são muito mais precisos que os anteriores, o que permite a obtenção de dados experimentais mais precisos, assim como a realização de experimentos que requerem um ajuste fino dos parâmetros.

A partir da relação de dispersão teórica (D1), são obtidas a velocidade de fase [72]

$$
v_{p}=\frac{\omega}{k}=c \operatorname{tg} \psi \sqrt{P_{1}},
$$

e a velocidade de grupo [72] 


$$
v_{g}=\frac{\partial \omega}{\partial k}=v_{p}+c k \operatorname{tg} \psi \frac{D P_{1}}{2 \sqrt{P_{1}}},
$$

onde $D P_{1}=\partial P_{1} / \partial k$. A Figura 21 mostra as velocidades de fase e de grupo em função da frequência da onda eletrostática. Observa-se na Figura 21 que a velocidade de fase decresce rapidamente para frequências entre 0 e $45 \mathrm{MHz}$. A velocidade de fase apresenta um mínimo em torno de $71 \mathrm{MHz}$ e depois desse ponto, ela se torna uma função ligeiramente crescente, com valor médio de $2,15 \times 10^{6} \mathrm{~m} / \mathrm{s}$ no intervalo entre 50 e $120 \mathrm{MHz}$. A velocidade de grupo também decresce rapidamente para baixas frequências. Entretanto, ela cresce consideravelmente depois de seu ponto de mínimo localizado ao redor de $27 \mathrm{MHz}$.

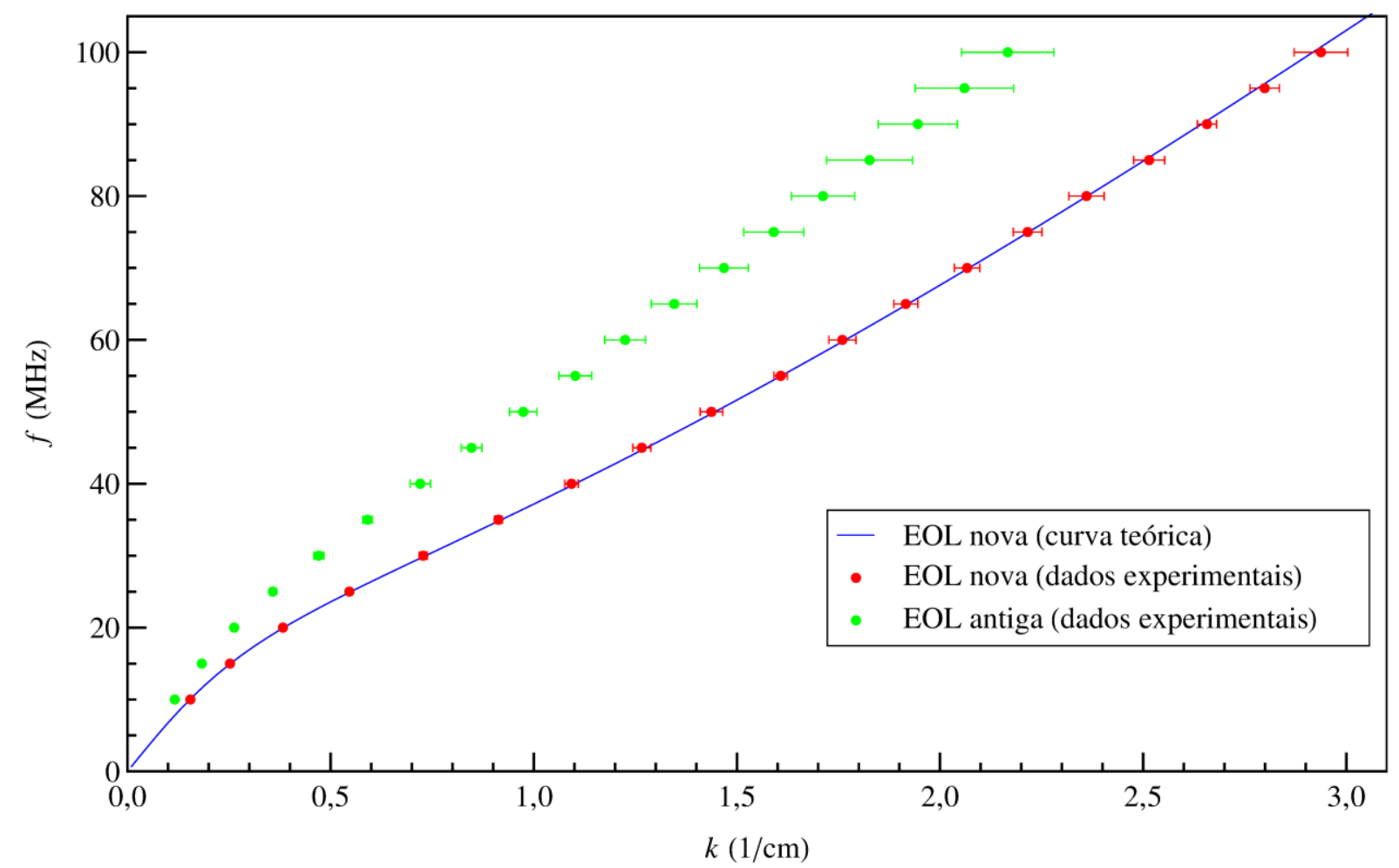

Figura 20: Relação de dispersão teórica (curva azul) e dados experimentais (em vermelho) para a nova versão da estrutura de ondas lentas (EOL). A figura também mostra uma comparação com os pontos experimentais (em verde) obtidos para a versão anterior do TWT.

Fonte: Referência [72]. 
(a) Velocidade de fase

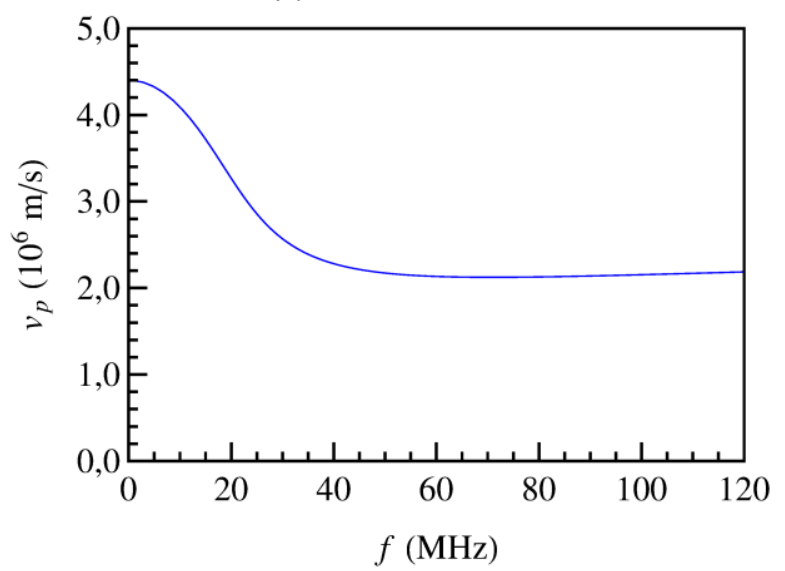

(b) Velocidade de Grupo

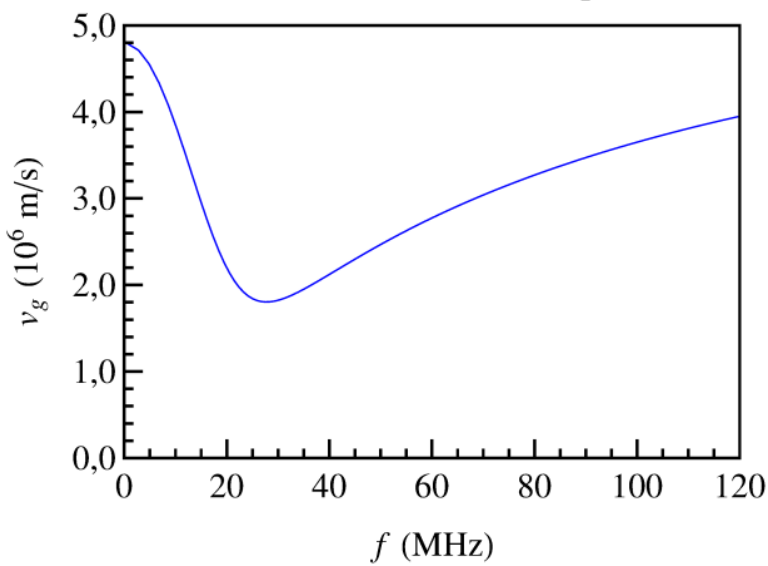

Figura 21: Velocidades de a) fase e de b) grupo em função da frequência da onda que se propaga no TWT. Fonte: Referência [72]. 